\title{
COMPARATIVE PROPERTIES OF WROUGHT IRON MADE BY HAND PUDDLING AND BY THE ASTON PROCESS
}

\author{
By Henry S. Rawdon and O. A. Knight
}

\section{ABSTRACT}

The hand-puddling method of making wrought iron has not greatly changed for a century. More economical methods in the manufacture of this product is the crying need of the industry. A radically new process, recently developed, is now coming into commercial use, in which pig iron, which has been refined in a Bessemer converter, is poured into molten slag so as to produce intimate mingling of the two. A comparison of the properties of wrought iron made thus with that made by hand puddling forms the subject of this report. The test results failed to show any marked difference in the products of the two processes. The new product appears to have all of the essential properties usually connoted by the name-wrought iron.

\section{CONTENTS}

I. Introduction

1. Résumé of the Aston process

II. Purpose and scope of the investigation 959

III. Materials and methods_._. 960

1. Materials_... 960

(a) Pipe

(b) "Rounds"

(c) Slag

2. Methods

IV. Results....... 962

1. Composition

2. Density _...... 964

3. Mechanical properties__. 965

(a) Pipe materials_._. 965

(1) Tensile properties_._. 965

(2) Torsional properties_._._. 970

(3) Flattening tests_._. 971

(b) 1-inch rounds _._. 972

(1) Tensile properties__._._. 972

(2) Torsional properties_._._. 973

(3) Impact resistance._._._._. 973

4. Corrosion resistance._.

(a) Laboratory corrosion tests_.............. 976

(b) Electrolytic solution potential__._._. 979

5. Structural examination

(a) Pipe materials

(1) Ball_...... 980

(2) Muck bar._._-_._.-. 980

(3) Skelp_........ 981

(4) Pipe

(b) 1-inch rounds

(c) Slag . 
IV. Results-Continued.

6. Miscellaneous tests and observations

(a) Observations on blistering

(b) Behavior on carburizing

(c) Mill observations

V. Discussion

VI. Summary

\section{INTRODUCTION}

The need for more economical methods in the manufacture of wrought iron has long been recognized. As stated by Aston, ${ }^{1}$ the outstanding needs of the wrought-iron industry are: Decreased cost of production and increased tonnage, the former being the more essential of the two. The realization of the importance of these needs is by no means new in the history of the industry. Shortly after the advent of the Bessemer process for manufacturing steel, attempts were started ${ }^{2}$ for increasing the scale of production of wrought iron and these attempts have continued down to the present day, some of the processes developed being used commercially with very considerable success. Practically all of the processes, with one notable exception, which have attained any degree of prominence or success attempted to duplicate in a mechanical way the operations of hand puddling, this being generally done by means of a movable furnace. The characteristic features of the present-day mechanical-puddling processes for the manufacture of wrought iron have been described in the technical literature, ${ }^{3}$ and further reference to them here is unnecessary. A few years ago a series of comparative tests was made by the Bureau of Standards of wrought iron made by one of these processes (footnote $3(c)$ ), together with wrought iron made by the same company by the usual hand-puddling method from pig iron of the same heat. One of the conclusions expressed as a result of these tests, a report of which has been published, ${ }^{4}$ was as follows:

The tests of two lots of wrought iron made by two distinctly different puddling processes have not shown anything to indicate that the hand-puddling product can not be equaled in an iron made by a mechanical-puddling process.

About a year ago the attention of this bureau was directed to the successful development, on a commercial scale, of a process for the production, by a very novel process, of a wrought iron which appears to possess the essential characteristics which are usually associated

1 James Aston, Trend of Development in the Wrought Iron Industry, Am. Inst. Min. Met. Eng., Pamphlet No. 1595-C; October, 1926.

.2 Thomas Turner, Metallurgy of Iron, 5th ed., p. 387, Charles Griffin \& Co.; 1918.

${ }^{3}$ Symposium on Wrought Iron: $(a)$ H. E. Smith, Manufacture and Use of Wrought Iron; (b) F. H. Dechant, Ely Process of Mechanical Puddling for the Production of Wrought Iron; (c) J. P. Roe, Roe Puddling Machine; (d) James Aston, Problem of Wrought Iron Manufacture and a New Process for Its Production, Am. Iron \& Steel Inst. Yearbook, 15, p. 117; 1925. See also footnote 1.

4 Henry S. Rawdon and Samuel Epstein, Observations on Phosphorus in Wrought Iron Made by Different Puddling Processes, Amer. Iron \& Steel Inst. Yearbook, 16, p. 117; 1926. 
with the time-honored product of the hand-puddling method. This new process differs radically in a good many respects from the conventional method of puddling. In this process, which will be referred to hereinafter as the "Aston process," after its inventor, Dr. James Aston, the essential objects accomplished in the puddling process as ordinarily understood, are attained in a unique manner. The melting of the raw material (pig iron) is done in a cupola, the refining of the molten metal in a Bessemer converter, the production of the slag of proper composition in an open-hearth furnace by itself, and the mechanical disintegration of the iron and the incorporation of the slag with it are attained by pouring a charge of the molten refined iron into a bath of the molten slag. The squeezing of the "ball" of iron into convenient form for working, although done somewhat differently from the method ordinarily employed with the hand-puddled product, involves no new principle, nor does the mechanical working of the squeezed iron into suitable form for handling in the subsequent operations.

This process has definitely passed the experimental stage and the product is now being produced on a tonnage basis, practically all of it being used in the manufacture of pipe. The United States Government is a purchaser of considerable amounts of wrought-iron pipe and has drawn up specifications ${ }^{5}$ covering such material. In view of this fact, therefore, together with the interest of the Bureau of Standards in obtaining information on new important industrial processes, a study of the properties of this material by the bureau was deemed both proper and desirable when the bureau was approached by the manufacturers in this matter. The study was undertaken on the research associate plan, ${ }^{6}$ the investigator appointed for carrying out the work being $\mathrm{O}$. A. Knight, associate professor of metallurgy, the Pennsylvania State College. The work was supported by the manufacturers, the A. M. Byers Co.

\section{RÉSUMÉ OF THE ASTON PROCESS}

This process differs from puddling, as ordinarily understood, in that the various objects to be accomplished are brought about as distinct steps carried out in separate furnaces or other units, instead of being a single-furnace process the nature of which gradually changes as it proceeds. The description of the operations given below is based upon observations made in the mill and represents the practice at the time the observations were made, but not necessarily all details as carried out at present. In melting the pig iron, which was of Bessemer grade, standard cupola melting practice was 
followed. Mention should be made of the treatment of the molten metal with soda ash to offset sulphur "pick-up" in the cupola. About 40 to 50 pounds of soda ash (anhydrous sodium carbonate) was placed in the ladle into which a charge of approximately 5,500 pounds of molten metal was tapped from the cupola. A period of about 15 minutes was required for this amount of metal to flow and this insured thorough mixing and plenty of time for the desulphurizing reactions to occur. The metal in the ladle was then skimmed free from slag and charged into the Bessemer converter.

A 2-ton side-blown converter was used, the "blow" usually lasting about 18 minutes. The composition of the blown metal is given in Table 1. After blowing, the metal was tapped into a lip-pour ladle and the slag raked off. No additions of manganese, silicon, aluminum, or other deoxidizing or degasifying agents, such as are used in steel manufacture, were employed. The metal was "shotted" in the condition as teemed from the converter. The ladle of metal was conveyed by means of a crane, which also carried scales for weighing the charge, to the "shotting cup" for the "shotting" operation, that is, the treatment of the metal with slag. Working drawings of one of the cups, which were made of cast iron and lined with fire brick, are given in Figure 1. A number of these cups, to be used in succession, are necessary in regular mill procedure.

TABLE 1.-Results of chemical analysis of metal at different stages of process ${ }^{1}$

\begin{tabular}{|c|c|c|c|c|c|c|c|c|c|c|c|c|c|c|c|}
\hline \multicolumn{5}{|c|}{ Cupola iron ${ }^{2}$} & \multirow{2}{*}{\multicolumn{5}{|c|}{ "Blown" metal" }} & \multirow{2}{*}{\multicolumn{6}{|c|}{ Wrought-iron muck, bars }} \\
\hline $\begin{array}{l}\text { Before } \\
\text { soda- } \\
\text { ash }\end{array}$ & \multicolumn{4}{|c|}{ After soda-ash addition } & & & & & & & & & & & \\
\hline $\mathrm{S}$ & Mn & $\mathrm{S}$ & $\mathbf{P}$ & $\mathrm{Si}$ & $\mathrm{C}$ & Mn & $\mathbf{S}$ & $\mathbf{P}$ & $\mathrm{Si}$ & $\mathrm{C}$ & Mn & $\mathbf{S}$ & $\mathbf{P}$ & $\mathrm{Si}$ & $\begin{array}{l}\text { Slag } \\
\text { and } \\
\text { oxides }\end{array}$ \\
\hline $\begin{array}{l}0.072 \\
.057 \\
.083 \\
.127 \\
.098\end{array}$ & $\begin{array}{r}0.56 \\
.49 \\
\\
\end{array}$ & $\begin{array}{l}0.039 \\
.039 \\
.044 \\
.044 \\
.048\end{array}$ & \begin{tabular}{c}
0.098 \\
.103 \\
\hdashline \\
-
\end{tabular} & $\begin{array}{l}1.44 \\
1.02 \\
1.09 \\
1.14 \\
1.13\end{array}$ & $\begin{array}{l}0.08 \\
.07 \\
.06 \\
.06 \\
.07\end{array}$ & $\begin{array}{r}0.02 \\
.04 \\
\\
\end{array}$ & $\begin{array}{r}0.048 \\
.040 \\
.057 \\
-.051\end{array}$ & $\begin{array}{r}0.100 \\
.103 \\
\end{array}$ & $\begin{array}{l}0.026 \\
.028 \\
.015 \\
.010 \\
.014\end{array}$ & $\begin{array}{r}0.02 \\
.03 \\
.03 \\
.02 \\
.03\end{array}$ & $\begin{array}{l}0.03 \\
.04 \\
.03 \\
.03 \\
.05\end{array}$ & $\begin{array}{c}0.031 \\
.027 \\
.037 \\
.042 \\
.042\end{array}$ & $\mid \begin{array}{r}0.100 \\
.097 \\
.118 \\
.112 \\
.130\end{array}$ & $\begin{array}{c}0.122 \\
.132 \\
.103 \\
.132 \\
.190\end{array}$ & $\begin{array}{l}2.46 \\
4.17 \\
2.98 \\
3.53 \\
4.25\end{array}$ \\
\hline $\begin{array}{l}.088 \\
.111 \\
.084 \\
.079\end{array}$ & & $\begin{array}{l}.056 \\
.050 \\
.040 \\
.040\end{array}$ & & $\begin{array}{r}1.19 \\
1.18 \\
.99 \\
.93\end{array}$ & $\begin{array}{l}.06 \\
.06 \\
.07 \\
.08\end{array}$ & & $\begin{array}{l}.060 \\
.060 \\
.046 \\
.048\end{array}$ & & $\begin{array}{l}.011 \\
.017 \\
.010 \\
.008\end{array}$ & $\begin{array}{l}.03 \\
.03 \\
.03 \\
.03\end{array}$ & $\begin{array}{l}.04 \\
.04 \\
.04 \\
.04\end{array}$ & $\begin{array}{l}.043 \\
.038 \\
.037 \\
.031\end{array}$ & $\begin{array}{l}.126 \\
.120 \\
.120 \\
.124\end{array}$ & $\begin{array}{l}.202 \\
.132 \\
.121 \\
.130\end{array}$ & $\begin{array}{l}4.30 \\
3.12 \\
3.08 \\
4.00\end{array}$ \\
\hline $\begin{array}{l}.079 \\
.091 \\
.100 \\
.110\end{array}$ & .51 & $\begin{array}{l}.035 \\
.043 \\
.042 \\
.044\end{array}$ & \begin{tabular}{c}
-.096 \\
\hdashline \\
\end{tabular} & $\begin{array}{l}1.18 \\
1.15 \\
1.02 \\
1.23\end{array}$ & $\begin{array}{l}.08 \\
.06 \\
.06 \\
.08\end{array}$ & .03 & $\begin{array}{l}.043 \\
.056 \\
.045 \\
.058\end{array}$ & .104 & $\begin{array}{l}.010 \\
.014 \\
.015 \\
.012\end{array}$ & $\begin{array}{l}.02 \\
.02 \\
.02 \\
.03\end{array}$ & $\begin{array}{l}.04 \\
.03 \\
.03 \\
.04\end{array}$ & $\begin{array}{l}.028 \\
.042 \\
.038 \\
.040\end{array}$ & $\begin{array}{l}.124 \\
.120 \\
.148 \\
.126\end{array}$ & $\begin{array}{l}.174 \\
.146 \\
.125 \\
.144\end{array}$ & $\begin{array}{l}4.94 \\
4.07 \\
3.32 \\
3.86\end{array}$ \\
\hline
\end{tabular}

1 The data given here were taken at random from the daily laboratory records of the A. M. Byers Co., covering a period of about 6 months at the mill at Warren, Ohio, by O. A. Knight.

2 All figures are in percentages.

Method described by Westcott, Eckert, and Einert, Ind. Eng. Chem., 19, p. 1285; 1927. 


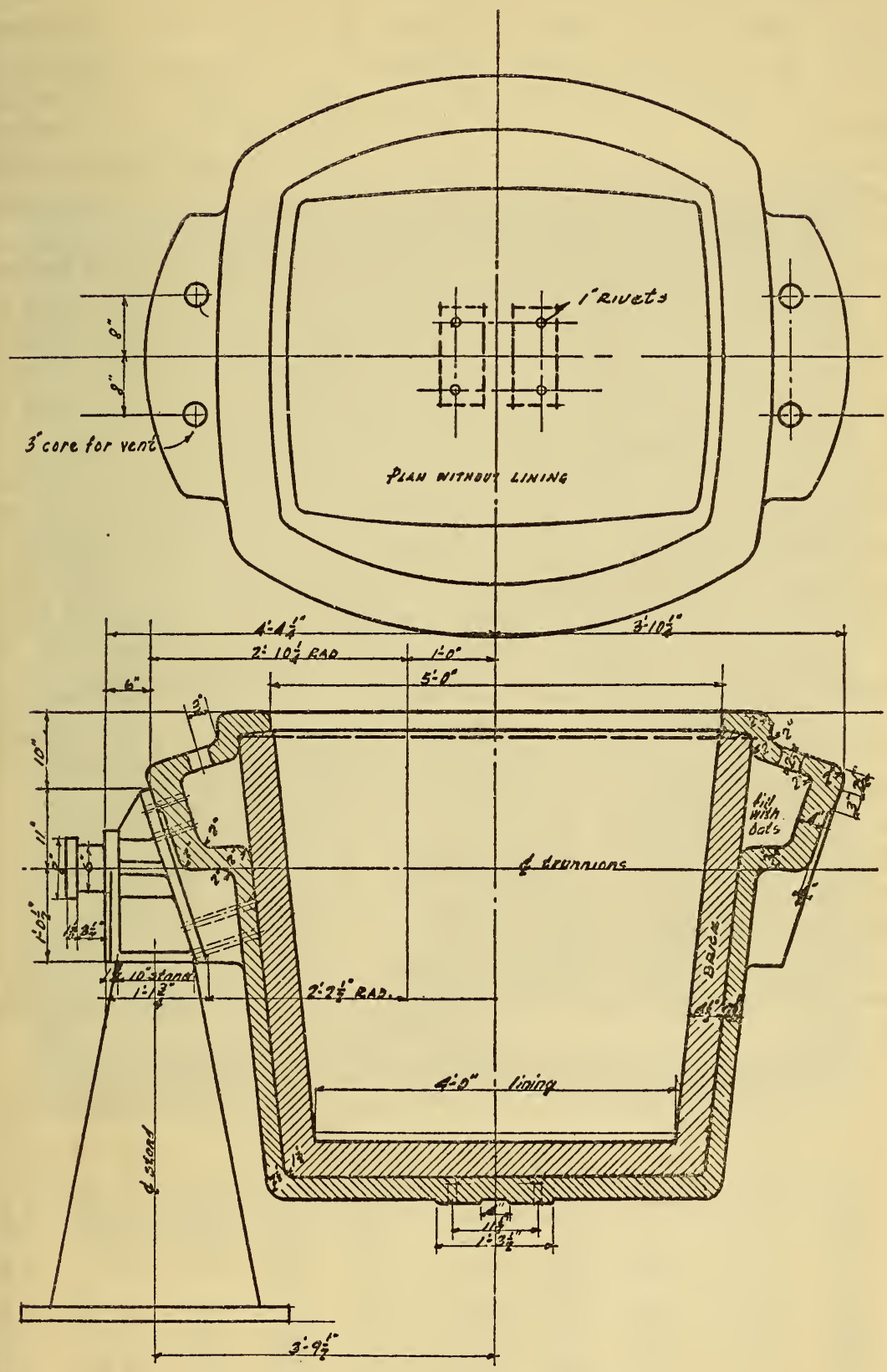

FIgURE 1.-Drawing of the shotting cup

Furnished by the manufacturers. 
The shotting operation was carried out as follows: (1) Molten slag was poured into one of the cups until it was slightly less than twothirds filled, (2) the ladle containing the blown metal was then moved up to this cup, and (3) molten metal was poured slowly over the lip of the ladle into the slag at a rate of about 1 ton in three minutes. (Fig. 2.) Only half of the charge of blown metal was poured into one cup, thus one ladle of blown metal furnished two balls, each weighing between 2,200 and 2,500 pounds. By observing the scale on the crane carrying the ladle a rather accurate division of the charge of the molten metal was possible. The height of the lip of the ladle above the slag surface in the cup was about 9 feet. The practice was followed of pouring about 800 pounds of the metal near one end of the cup, a like amount near the other end, and the remainder near the middle of the cup. The temperature of the molten slag must be maintained considerably below that of the molten iron which is to be poured into it. If no precautions were taken the temperature of the slag might rise sufficiently, as a result of the heat liberated by the iron as it passes from the liquid state upon quenching, to interfere seriously with the efficacy of the slag as a quenching medium. An undue rise of temperature of the slag was prevented by throwing a shovelful of cold granulated slag into the shotting cup from time to time. This also served to prevent "boiling over" of the slag in the cup, which might otherwise occur as a result of the vigorous gas evolution which takes place when the stream of molten metal is poured into the molten slag. During the course of the shotting operation the cup had the appearance of being full. However, the boiling subsided quickly as soon as the last of the molten metal had been added and, when quiet, the cup was only slightly more than two-thirds full. The excess slag was then decanted into the cup which was to be used next (fig. 3), and the "ball" was dumped onto a carriage (fig. 4) and was ready to be taken to the press.

TARLE 2.-Results of chemical analysis of shotting slag 1

\begin{tabular}{|c|c|c|c|c|c|c|c|c|c|}
\hline \multicolumn{4}{|c|}{ Before shotting 2} & \multicolumn{6}{|c|}{ After shotting 2} \\
\hline $\mathrm{SiO}_{2}$ & $\mathrm{Fe}$ & $\mathrm{FeO}$ & $\mathrm{Fe}_{2} \mathrm{O}_{3}$ & $\mathrm{SiO}_{2}$ & Mn & $\mathbf{P}$ & $\mathrm{Fe}$ & $\mathrm{FeO}$ & $\mathrm{Fe}_{3} \mathrm{O}_{3}$ \\
\hline $\begin{array}{l}8.04 \\
8.56\end{array}$ & $\begin{array}{l}66.46 \\
65.15\end{array}$ & $\begin{array}{l}78.93 \\
71.69\end{array}$ & $\begin{array}{r}7.36 \\
13.44\end{array}$ & $\begin{array}{l}7.78 \\
8.90\end{array}$ & $\begin{array}{r}0.96 \\
.92\end{array}$ & $\begin{array}{r}0.197 \\
.200\end{array}$ & $\begin{array}{l}66.36 \\
64.94\end{array}$ & $\begin{array}{l}77.98 \\
74.55\end{array}$ & $\begin{array}{l}8.38 \\
9.97\end{array}$ \\
\hline $\begin{array}{r}7.80 \\
10.92 \\
8.56\end{array}$ & $\begin{array}{l}65.66 \\
65.06\end{array}$ & $\begin{array}{l}71.31 \\
70.49\end{array}$ & $\begin{array}{l}14.60 \\
14.64\end{array}$ & $\begin{array}{r}9.32 \\
11.08 \\
10.58\end{array}$ & $\begin{array}{l}.90 \\
.92 \\
.88\end{array}$ & $\begin{array}{l}.235 \\
.352 \\
.336\end{array}$ & $\begin{array}{l}65.07 \\
64.07 \\
62.85\end{array}$ & $\begin{array}{l}72.03 \\
75.68 \\
71.78\end{array}$ & $\begin{array}{r}12.96 \\
7.46 \\
10.05\end{array}$ \\
\hline $\begin{array}{r}10.28 \\
10.00 \\
10.26 \\
9.30 \\
10.12\end{array}$ & $\begin{array}{l}66.84 \\
64.09 \\
62.51 \\
63.93 \\
64.60\end{array}$ & $\begin{array}{l}70.32 \\
70.32 \\
70.00 \\
65.93 \\
69.31\end{array}$ & $\begin{array}{l}17.37 \\
13.46 \\
11.55 \\
17.96 \\
15.30\end{array}$ & $\begin{array}{l}12.88 \\
10.54 \\
10.36 \\
10.42 \\
10.34\end{array}$ & $\begin{array}{r}1.16 \\
.96 \\
1.05 \\
1.03 \\
1.13\end{array}$ & $\begin{array}{l}.440 \\
.384 \\
.420 \\
.364 \\
.424\end{array}$ & $\begin{array}{l}63.21 \\
63.90 \\
62.12 \\
63.20 \\
63.90\end{array}$ & $\begin{array}{l}73.10 \\
73.73 \\
72.79 \\
70.13 \\
72.40\end{array}$ & $\begin{array}{r}9.11 \\
9.38 \\
7.89 \\
12.40 \\
10.87\end{array}$ \\
\hline $\begin{array}{r}9.00 \\
9.74\end{array}$ & 65.40 & 65.84 & 20.31 & $\begin{array}{r}10.48 \\
9.98\end{array}$ & 1.10 & .440 & 64.10 & 69.83 & 14. 01 \\
\hline $\begin{array}{l}10.16 \\
10.10\end{array}$ & $\begin{array}{l}66.80 \\
65.96\end{array}$ & $\begin{array}{l}72.92 \\
63.69\end{array}$ & $\begin{array}{l}14.44 \\
23.38\end{array}$ & $\begin{array}{r}9.98 \\
10.86 \\
11.78\end{array}$ & $\begin{array}{l}1.03 \\
1.07\end{array}$ & $\begin{array}{l}.380 \\
.392\end{array}$ & $\begin{array}{l}65.30 \\
63.69\end{array}$ & $\begin{array}{l}74.43 \\
69.26\end{array}$ & $\begin{array}{l}10.73 \\
14.04\end{array}$ \\
\hline
\end{tabular}

1 The data given here were obtained in the same manner as those in Table 1.

${ }^{2}$ All results are in percentages. 
B. S. Journal of Research, RPI24

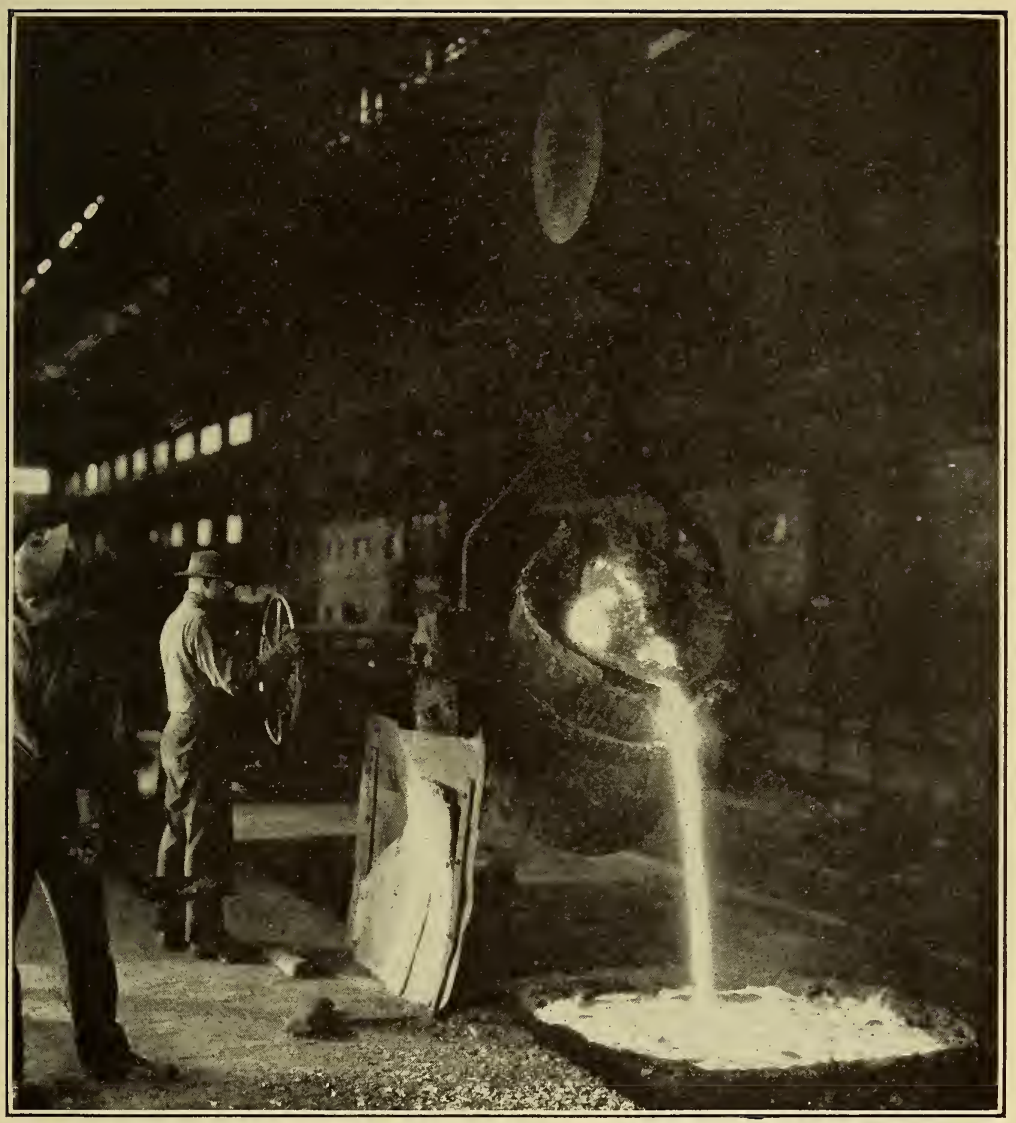

FIGURE 2.-The shotting operation

The metal in the tilted ladle has just come from the Bessemer converter where it has been "blown." Note the surface of the slag in the lower container, the shotting cup, which nearly reaches the top of the cup on account of the vigorous gas evolution produced by pouring the molten metal into the molten slag. 
B. S. Journal of Research, RP124

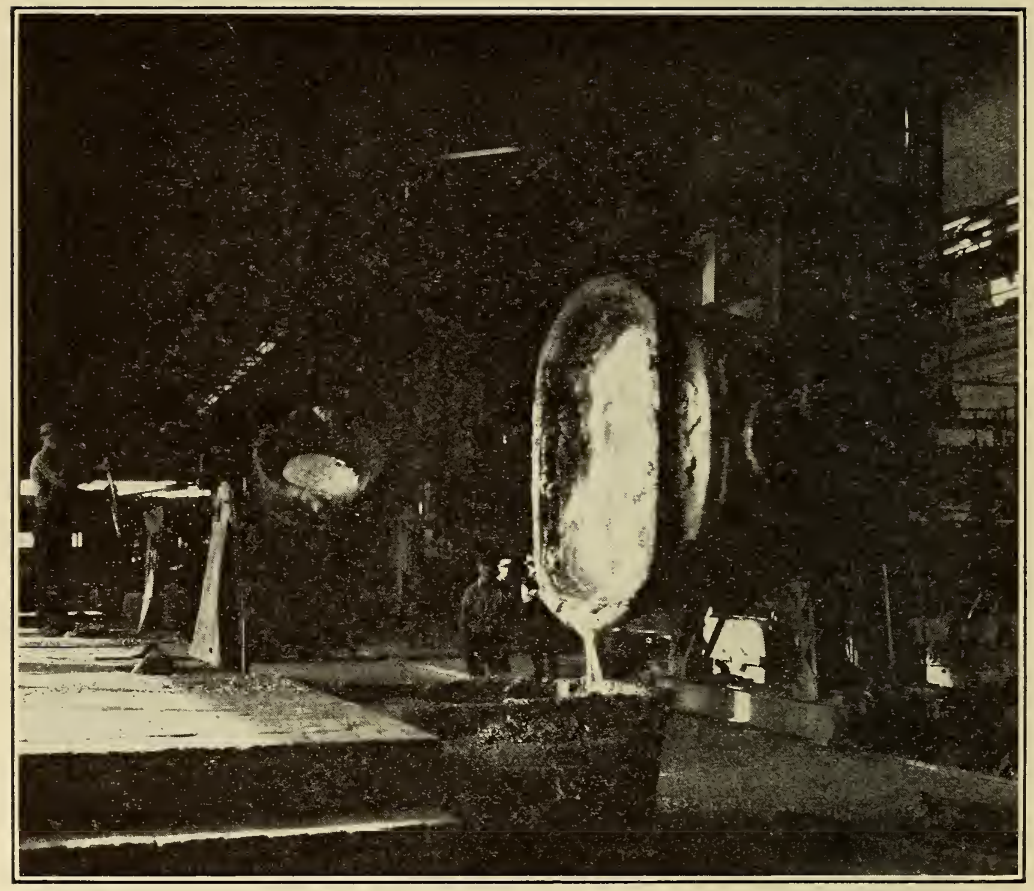

FIGURE 3.-Decanting the excess slag after the shotting operation

The slag is poured into another cup to be used for shotting the next ladle of metal. Note the ball within the upper cup. 
B. S. Journal of Research, RPI24

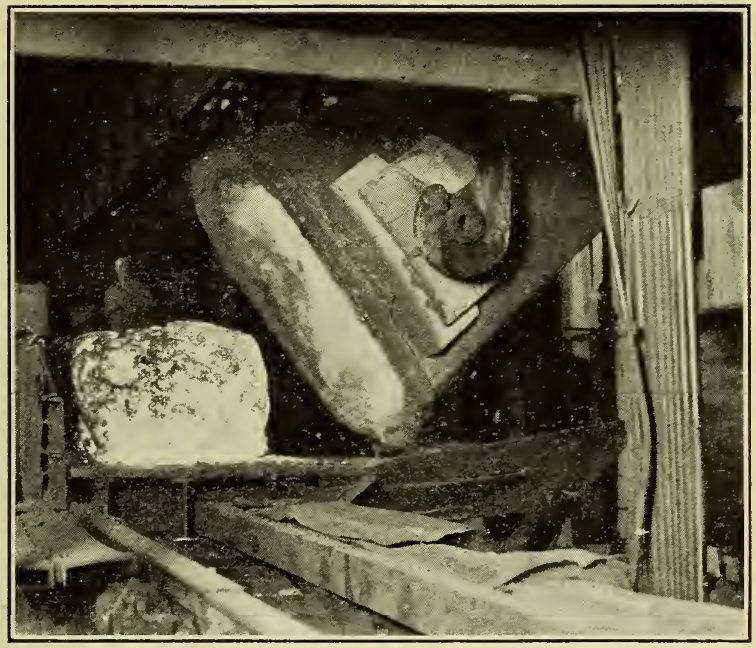

FIGURE 4.-The ball after being dumped from the shotting cup

It is now ready for the squeezing operation.

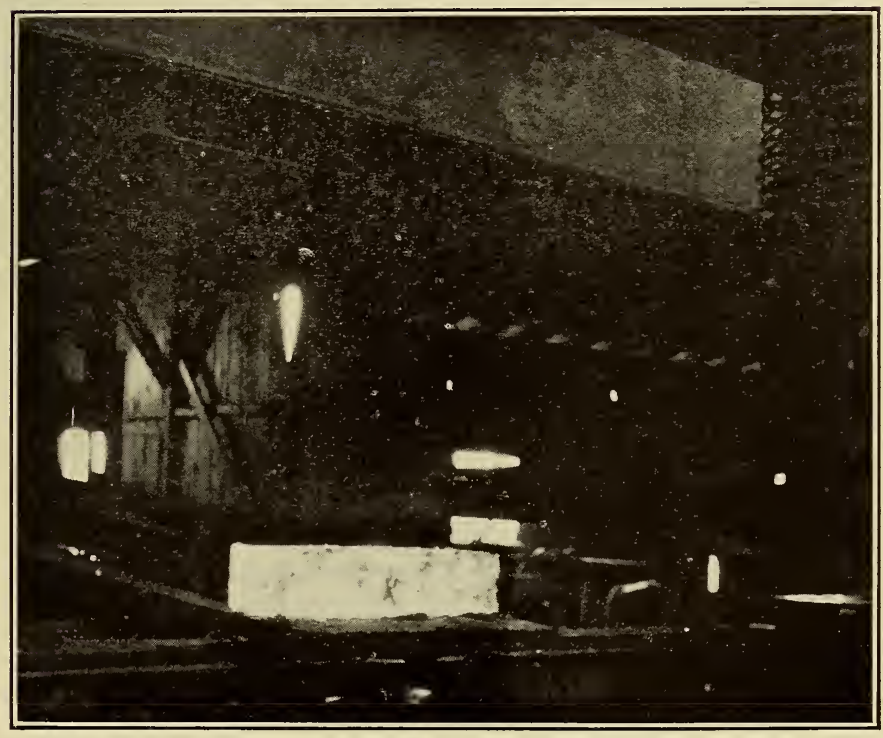

FIGURE 5.-The bloom as it comes from the press

Much of the slag has been squeezed out and the material is quite compact. It is now ready for the rolling mill where it will be rolled into muck bar. 
The slag into which the metal was poured in the shotting operation was prepared in a 15-ton tilting open-hearth furnace. The composition of this slag was close to the average composition of that which is formed in ordinary puddling. (Table 2.) The effect produced by the shotting operation evidently depends mainly upon the difference in temperature between metal and slag and the evolution of gas which occurs when the two materials are brought together. Previous observations in the mill had indicated that the temperature of the molten slag within the cup was somewhat above $1,315^{\circ} \mathrm{C} .\left(2,400^{\circ} \mathrm{F}\right.$.), whereas the temperature of the molten metal was considerably higher, being approximately $1,540^{\circ}$ to $1,590^{\circ} \mathrm{C}$. $\left(2,800^{\circ}\right.$ to $2,900^{\circ} \mathrm{F}$.). The sudden violent evolution of gas which occurs as the metal is poured into the slag comminutes it into a relatively fine state of division, thus facilitating the incorporation of the slag within the metal. The particles of metal accumulate in the lower part of the cup and agglomerate into a coherent spongy mass or "ball" resembling in all essential respects, except size, that taken from the puddling furnaces.

The squeezing of the ball to free it from excess slag did not differ in principle from the similar operation in connection with ordinary puddling. In this case, however, a hydraulic press which squeezed the ball on the four sides and the ends was used. A fairly compact bloom measuring approximately 12 by 14 inches by 5 feet was the result. The bloom is shown in Figure 5 on its way to the rolling mill, where it was given nine passes in a 29-inch mill and seven in a 24-inch mill which reduced it to the form of muck bar three-fourths inch thick by 8 inches wide. The composition of the muck bar is given in Table 1. The lower carbon content of the muck bar as compared to the blown metal is to be attributed to the effect of the shotting. The increase in the silicon content is, of course, the result of the mechanically included slag. The effect of the desulphurizing action of the soda-ash treatment of the cupola metal is unmistakable. It would appear also that a slight decrease in sulphur content accompanies the shotting operation.

\section{PURPOSE AND SCOPE OF THE INVESTIGATION}

As stated above, iron made by the Aston process is now a commercial product, being sold under the same designation as is the product of the older and more familiar puddling process, and intended for the same usage. It has been tacitly assumed by the manufacturers that the two materials are not essentially different and that the newer product, regardless of the novel features of its method of production, is fully entitled to the name, "wrought iron."

The matter of the definition of wrought iron; that is, whether this material should be defined mainly in terms of the process by 
which it has been produced or by the properties which it possesses is of some importance in the preparation of specifications. It was considered, however, that a determination of the properties of the new wrought iron, particularly as compared with those of wrought iron made by the hand-puddling process, was a matter for more immediate consideration. This was made the main purpose of the investigation, therefore, and this report summarizes the results obtained in a comparison of the properties of the two types of material. Both materials are referred to herein as "wrought iron" for convenience in discussion. It is believed that the question of the inclusion of the new product under the term "wrought iron" in specifications is a matter that will automatically settle itself one way or the other in accordance with the degree of similarity of the two types of materials.

\section{MATERIALS AND METHODS}

\section{MATERIALS}

(a) PIPE

Since iron made by the Aston process is being used commercially only in the form of pipe, the majority of the tests which were carried out were on pipe materials. In addition to the tests of the finished pipe, 1-inch butt-welded pipe being used throughout, similar tests were made on the pipe skelp, and on the muck bar, or "first rolling," which is the product which is sheared into short lengths, piled together, reheated, and rolled into the form of skelp. Some observations were also made upon the ball before it had been subjected to any working whatsoever. In all cases the tests were carried out on both hand-puddled iron and on the new product, the two materials being the products of the same manufacturer. In the case of skelp and pipe, similar tests were_also made on a composite iron made by using 50 per cent of each of the two irons in the form of sheared muck bar in forming the "pile" which was heated and rolled into the form of skelp. The pile was similar to a sandwich in that the hand-puddled iron formed the surface layers and the new-process iron the central part. All samples used throughout were taken under the supervision of the authors and represent "run-of-mill" material, unless stated otherwise.

In selecting the samples of muck bar and skelp, four different strips were chosen at random, as they were rolled, and pieces were cut from three positions along the length representative of the "front," "middle," and "back" of the strip as rolled. The purpose 
of using three samples from a single strip was to show the degree of uniformity of the material. The pipe samples were 10 -foot lengths of commercial black pipe of the three kinds-hand-puddled iron, new-process, and the composite iron formed of equal parts of the other two. Three samples of each kind of pipe were used, the selection in each case being made at random.

The identification mark, which was stamped on each sample at the mill, was chosen so as to show (a) the nature of the iron (HP, handpuddled iron; A, iron made by the Aston process; AHP, the composite iron); (b) the position of the sample in the strip, skelp, or muck bar, as rolled ( $\mathrm{F}$, front; $\mathrm{M}$, middle, $\mathrm{B}$, back); and (c) the number of the sample strip or pipe $(1,2,3,4)$. Thus, for example, "HPF1," as relating to skelp indicates the piece cut from the front end of the hand-puddled skelp strip No. 1. These designations will be used in summarizing the test results later in this report.

(b) ROUNDS

In addition to the tests on pipe materials, some work was done on 1-inch rounds of the new wrought iron. Although the new product is not being, used commercially in this form, as yet, wrought iron in this form is widely used. These rounds were prepared especially for this work. By using material in this form it was possible to carry out a number of additional tests which were not practicable with the pipe materials. A rather extensive series of tests on 1-inch rounds of high-grade hand-puddled wrought iron made by several different manufacturers has already been carried out by the bureau, ${ }^{7}$ and the results of these tests were used for comparison with those obtained with the new iron.

Three lots of 1-inch rounds were rolled from the same bloom, 11/1/2 by 13 inches in cross section, the amount of working received being different for the three. In the preparation of one lot, which will be referred to as "direct rolled," the bloom was rolled, without reheating, into a billet, 4 by $4 \frac{1}{2}$ inches in section, a part of which, after reheating, was rolled directly into the form of 1 -inch rounds. In the preparation of the other two lots the rolling of the billet was carried far enough to produce muck bar, $3 / 4$ by $4 \frac{1}{2}$ inches in section, which was then sheared into shorter lengths, assembled into 8-high piles and reheated. One pile was rolled directly into 1-inch rounds, which will be referred to as "single refined." Another 8-high muck-bar pile, after reheating, was rolled into bar form, $3 / 8$ by $4 \frac{1}{2}$ inches in section. This, in turn, was sheared into short lengths, assembled

${ }^{7}$ H. S. Rawdon and S. Epstein, The Nick-Bend Test for Wrought Iron, B. S. Tech. Paper No. 252; 1924. Proc. Am. Soc. Test. Mtls., 22, Pt. II, p. 193; 1922. 
into an 8-high pile, reheated, and rolled into 1-inch rounds. This lot of material will be referred to as "double refined."

\section{(c) SLAG}

The observations which were made upon the slag were on rather large masses of the slag as well as upon slag recovered from the finished pipe. In the case of the Aston process, a small "ingot" of slag was poured into a chill mold; whereas in the puddling process a rather large piece was raked out of the furnace. Observations were also made on samples obtained by collecting the slag as it dripped from the ball.

\section{METHODS}

In addition to the test methods which are commonly found in specifications for wrought-iron pipe and bar stock, a number of other tests were used, which, it was thought, might reveal important differences in the materials if they existed. Chemical analyses were made of both iron and slag. The physical testing methods used included density determinations as well as the usual tension, torsion, impact, and flattening tests. The structural examination of the materials, from both the microscopic and the macroscopic aspect, constituted an important part of the study. Laboratory corrosion tests were carried out on the finished pipe by various accelerated methods in both tap water and sea-salt solution, together with some observations on the relative electrolytic solution potentials of the two types of iron. Observations were also made in the mill for the purpose of comparing the behavior of the irons in welding, galvanizing, and machining.

\section{RESULTS}

\section{COMPOSITION}

The samples used for chemical analysis consisted of short lengths from each end of three 10-foot lengths of the new-process iron pipe, from each end of a similar length of hand-puddled iron pipe, and from each end of a similar length of pipe made of the composite iron. The results are given in Table 3. It is apparent from these results that there is no marked difference in composition between iron made by hand puddling and that made by the new process. The carbon and phosphorus contents were found to be consistently lower in the new iron than in the hand-puddled product, but the sulphur content was somewhat higher. The latter was lower than the average shown by the mill analyses made on muck bar (Table 1), as was the carbon content also. 
TABLE 3.-Results of chemical analysis of the three types of pipe used 1

[ $\mathrm{A}=$ new -process iron, $\mathrm{HP}=$ hand-puddled, $\mathrm{AHP}=$ composite iron; $\mathrm{x}$ and $\mathrm{y}$ indicate the two ends of the pipe; 1,2 , or 3 indicate the number of the pipe]

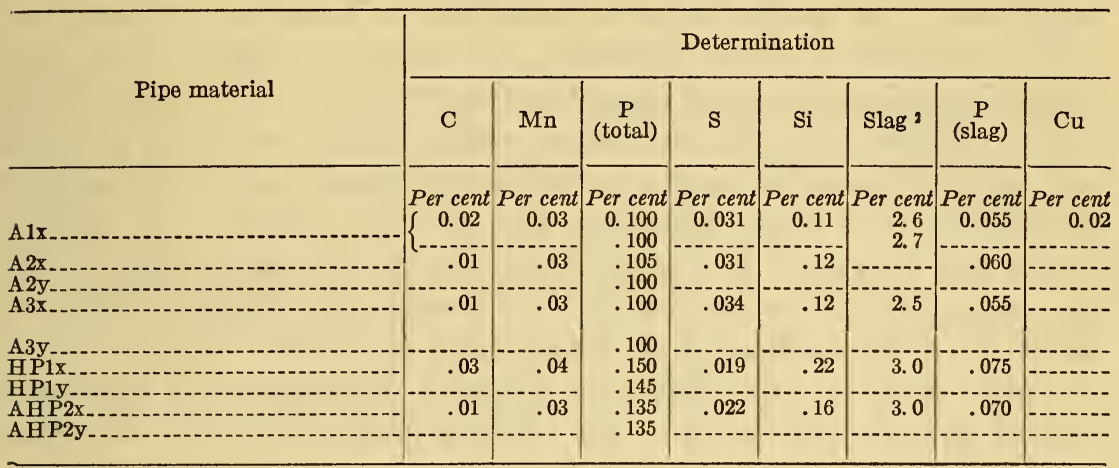

1 Analysis by H. A. Bright, associate chemist, Bureau of Standards

Iodine _method. See Proc. Am. Soc. Test. Materials, 25, pt. 1, p. 79; 1925.

The chemical composition of the slag used in the Aston process and that produced in the hand-puddling process was determined. In each case a large lump of slag, as stated above, was used for sampling, as well as slag drippings from the wrought-iron ball. In addition, analyses were made of the slag recovered by dissolving a sample of the pipe in an iodine-potassium-iodide solution. The results are given in Table 4.

TABLE 4.-Chemical composition of slag ${ }^{1}$

\begin{tabular}{|c|c|c|c|c|c|c|c|}
\hline \multirow{2}{*}{ Material } & \multicolumn{7}{|c|}{ Determination } \\
\hline & $\mathrm{FeO}$ & $\mathrm{Fe}_{2} \mathrm{O}_{3}$ & $\mathrm{SiO}_{2}$ & $\mathrm{P}_{2} \mathrm{O}_{5}$ & $\mathrm{MnO}$ & $\mathrm{CaO}$ & $\mathrm{Al}_{2} \mathrm{O}_{3}$ \\
\hline Slag, Aston process, lump & $\begin{array}{c}\text { Per cent } \\
71.0\end{array}$ & $\begin{array}{c}\text { Per cent } \\
10.3\end{array}$ & $\begin{array}{c}\text { Per cent } \\
12.0\end{array}$ & $\begin{array}{c}\text { Per cent } \\
1.2\end{array}$ & Per cent & Per cent & Per cent \\
\hline Same, recovered from the pipe & 57.0 & 25.0 & 9.0 & 2.0 & 1.4 & 0.4 & $1 . \overline{2}$ \\
\hline $\begin{array}{l}\text { Same, obtained as "drippings from the } \\
\text { ball" }\end{array}$ & 70.7 & 8.9 & 11.6 & 1.34 & 1.76 & & \\
\hline Slag, hand-puddiling process, lump........ & 66.2 & $\begin{array}{l}8.9 \\
7.2\end{array}$ & 14. 8 & 3.1 & 1.60 & & 2.0 \\
\hline Same, recovered from the pipe ${ }^{2}$ & 63.0 & 12.0 & 14.0 & 3.0 & 1.8 & $<1.0$ & 5.0 \\
\hline $\begin{array}{l}\text { Same, , obtained as "drippings from the } \\
\text { ball" }\end{array}$ & & 14.5 & 11.5 & 1.56 & 1.55 & & 2.4 \\
\hline
\end{tabular}

1 Analysis by H. A. Bright and C. P. Larrabee, Bureau of Standards.

2 The slag was separated from the pipe by the iodine method.

The composition of the slag used in the new process, as determined on a lump sample, agreed well with the average composition shown by the mill laboratory records. The differences in composition of the two types of slags (as determined on comparable samples) were slight. Too much significance should not be attached to the apparent difference in composition of the slags determined on the residues obtained by dissolving a sample of the finished pipe as compared with the same slags in lump form. The $\mathrm{Fe}_{2} \mathrm{O}: \mathrm{FeO}_{3}$ ratio of slag recovered as a residue after dissolving the iron may be altered as a result of the method used in recovering the slag. 


\section{DENSITY}

The density of the three irons in various stages of working was determined, the results being summarized in Table 5. On account of the porosity of these materials, the results should not be considered as indicating in all cases the true density. The results agree well, however, with other published results for the density of wrought iron and are believed to give reliable indications as to the relative densities of the three materials and of the variations in density in any one product. It will be noted that the average density of the iron made by the Aston process was consistently slightly higher in all of the products than that of the other two irons. In the case of the pipe, however, the difference between maximum and minimum for the new-process material was slightly greater than for the other pipe.

TABLE 5.-Density of wrought iron in various stages of working ${ }^{1}$

( $\mathrm{A}=$ iron made by Aston process; $\mathrm{H} \mathrm{P}=$ hand-puddled iron; $\mathrm{AH} \mathrm{P}=$ composite iron containing 50 per cent of each $A$ and $H P$. F, M, and $B=$ front, middle, and back, respectively, $x$ and $y$ indicate the two ends of the pipe length]

\begin{tabular}{|c|c|c|c|}
\hline \multicolumn{3}{|c|}{ Material } & \multirow{2}{*}{$\begin{array}{l}\text { Density, } \\
\text { g/cm } \mathrm{cm}^{3}, \\
25^{\circ} \mathrm{C} .\end{array}$} \\
\hline Process & Rolled product & Sample & \\
\hline \multirow{2}{*}{ Aston.- } & \multirow{3}{*}{ Muck bar.... } & $\left(\begin{array}{l}\text { AF1 } 1 \\
\text { AM1 } \\
\text { AB1 }\end{array}\right.$ & $\begin{array}{l}7.63 \\
7.65 \\
7.58\end{array}$ \\
\hline & & $\begin{array}{l}\text { AF4 } \\
\text { AM4 } \\
\text { AB4 }\end{array}$ & $\begin{array}{l}7.61 \\
7.57 \\
7.65\end{array}$ \\
\hline \multirow[t]{2}{*}{ A verage . - } & & & 7.615 \\
\hline & \multirow{3}{*}{ Muck bar } & {$\left[\begin{array}{l}\mathrm{H} P \mathrm{P} 1 \\
\mathrm{H} P \mathrm{P} 1 \\
\mathrm{H} \mathrm{PB} 1\end{array}\right.$} & $\begin{array}{l}7.53 \\
7.55 \\
7.60\end{array}$ \\
\hline Hand-puddled.... & & $\mid \begin{array}{l}\text { H PF2 } \\
\text { HPM2-- } \\
\text { H PB2 }\end{array}$ & $\begin{array}{l}7.61 \\
7.62 \\
7.64\end{array}$ \\
\hline A verage ............ & & & 7.59 \\
\hline \multirow{2}{*}{ A.ston } & \multirow{3}{*}{ Skelp_........ } & $\left\{\begin{array}{l}\text { AF3 } \\
\text { AM3 } \\
\text { AB3 }\end{array}\right.$ & $\begin{array}{l}7.67 \\
7.66 \\
7.66\end{array}$ \\
\hline & & $\begin{array}{l}\text { AF4 } \\
\text { AM4 } \\
\text { AB4 }\end{array}$ & $\begin{array}{l}7.68 \\
7.67 \\
7.68\end{array}$ \\
\hline A verage ... & & & 7.67 \\
\hline \multirow{3}{*}{ Hand-puddled...- } & \multirow{3}{*}{ Skelp... } & $\left\{\begin{array}{l}\text { HPF1 } \\
\text { HPM1 } \\
\text { HPB1 }\end{array}\right.$ & $\begin{array}{l}7.64 \\
7.65 \\
7.67\end{array}$ \\
\hline & & $\begin{array}{l}\text { HPF4 } \\
\text { HPM4 } \\
\text { HPB4 }\end{array}$ & $\begin{array}{l}7.64 \\
7.63 \\
7.66\end{array}$ \\
\hline & & & 7.65 \\
\hline
\end{tabular}

1 Density measurements by E. L. Peffer, physicist, Bureau of Standards. 
TABLE 5.-Density of wrought iron in various stages of working-Continued

\begin{tabular}{|c|c|c|c|}
\hline \multicolumn{3}{|c|}{ Material } & \multirow{2}{*}{$\begin{array}{l}\text { Density, } \\
\text { g/cm } \\
25^{\circ} \mathrm{C}\end{array}$} \\
\hline Process & Rolled product & Sample & \\
\hline \multirow{2}{*}{ Composite..... } & \multirow{3}{*}{ Skelp_-_. } & $\left\{\begin{array}{l}\text { AHPF1-.- } \\
\text { AH PM1 } \\
\text { AHPB1 }-\end{array}\right.$ & $\begin{array}{l}7.61 \\
7.64 \\
7.64\end{array}$ \\
\hline & & $\begin{array}{l}\text { AH PM4 } \\
\text { AHPB4 }\end{array}$ & $\begin{array}{l}7.64 \\
7.63 \\
7.64\end{array}$ \\
\hline \multirow[t]{2}{*}{ A verage - . } & & & 7. 63 \\
\hline & \multirow{3}{*}{ Pipe....... } & $\left(\begin{array}{l}\text { Alx } \\
\text { A1y }\end{array}\right.$ & $\begin{array}{l}7.68 \\
7.66\end{array}$ \\
\hline \multirow[t]{2}{*}{ Aston } & & $\left\{\begin{array}{l}\mathrm{A} 2 \mathrm{x} \\
\mathrm{A} 2 \mathrm{y}\end{array}\right.$ & $\begin{array}{l}7.66 \\
7.65\end{array}$ \\
\hline & & $\mid \begin{array}{l}\text { A3x----.-- } \\
\text { A3y-.--.. }\end{array}$ & $\begin{array}{l}7.61 \\
7.64\end{array}$ \\
\hline \multirow[t]{2}{*}{ Average } & & & 7.65 \\
\hline & \multirow{4}{*}{ Pipe-1 } & $\left(\begin{array}{l}\mathrm{HP} P \mathrm{H} \\
\mathrm{H} P 1 \mathrm{y}\end{array}\right.$ & $\begin{array}{l}7.62 \\
7.62\end{array}$ \\
\hline \multirow[t]{2}{*}{ Hand-puddled .- } & & $\left\{\begin{array}{l}\text { HP2x } \\
\text { HP2y }\end{array}\right.$ & $\begin{array}{l}7.63 \\
7.61\end{array}$ \\
\hline & & $\mid \begin{array}{l}\text { HP3x } \\
\text { H P3y }\end{array}$ & $\begin{array}{l}7.61 \\
7.62\end{array}$ \\
\hline \multirow[t]{2}{*}{ A verage...- } & & - & 7.62 \\
\hline & \multirow{4}{*}{ Pipe...... } & $\left(\begin{array}{l}\text { AHP1x } \\
\text { AHP1y.-. }\end{array}\right.$ & $\begin{array}{l}7.61 \\
7.62\end{array}$ \\
\hline \multirow[t]{2}{*}{ Composite-...... } & & $\left\{\begin{array}{l}\text { AНP2x }-. . \\
\text { АН } 2 \text { Р }\end{array}\right.$ & $\begin{array}{l}7.61 \\
7.61\end{array}$ \\
\hline & & $\mid$ & $\begin{array}{l}7.62 \\
7.63\end{array}$ \\
\hline A verage..... & & & 7.62 \\
\hline
\end{tabular}

\section{MECHANICAL PROPERTIES}

(a) PIPE MATERIALS

The behavior of the three irons, in the form of muck bar, skelp, and pipe was determined under tension. Torsion tests and also flattening tests were made of the finished pipe.

(1) Tensile Properties.-The tensile properties of the iron in the form of muck bar (one-half inch thick) were determined on 20-inch specimens with a reduced section $1 \frac{1}{2}$ inches wide. The elongation was measured over both an 8-inch and a 2-inch length. The skelp specimens were of the same size, except in thickness which was that of the skelp strip as rolled. This was approximately 0.133 inch, including the surface scale $(0.03$ inch), which flaked off during the test. All of the tests were made with an Amsler tension testing machine of 50,000 pounds capacity. For a number of the specimens of each of the three irons, the Ewing extensometer was used in order to obtain the necessary data for the stress-strain curves. These curves for the muck bar and skelp are given in Figures 6 and 7 , respectively. 


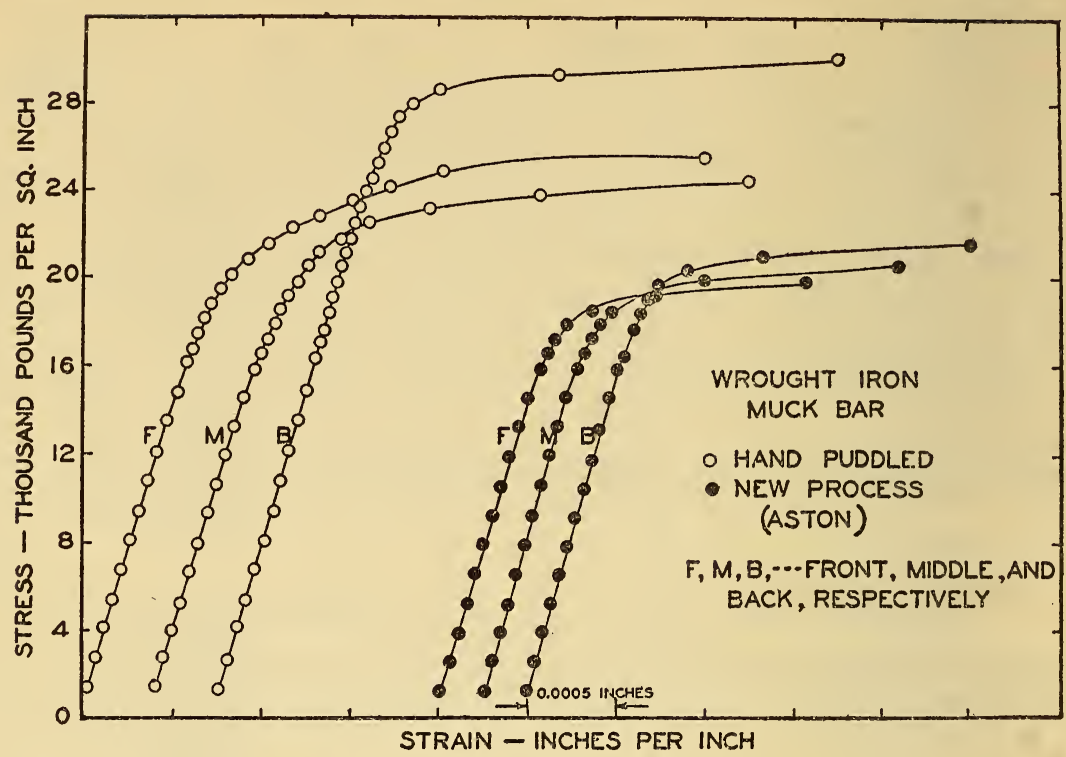

Figdre 6.-Stress-strain curves of wrought iron in the muck-bar condition tested in tension

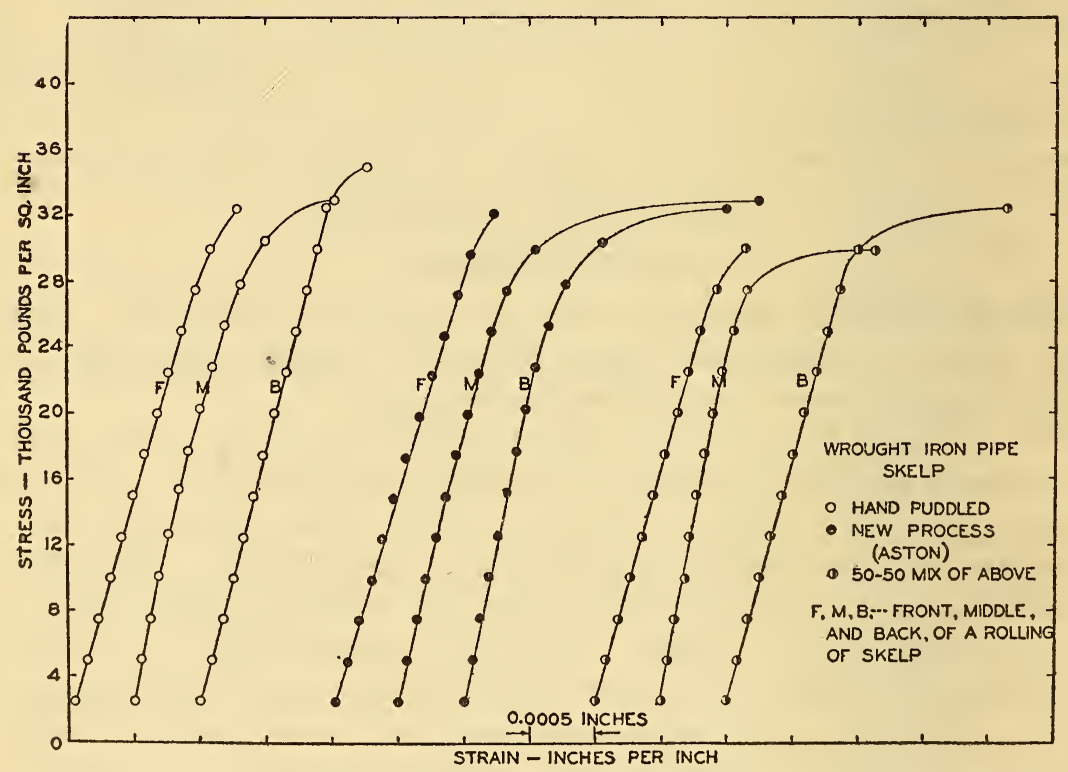

Figure 7.-Stress-strain curves of wrought iron in the form of pipe skelp tested in tension 
The tensile properties of the two irons in the muck-bar state are summarized in Table 6 and of the skelp in Table 7. Here and elsewhere, the test results have been "rounded off" to the nearest 50 pounds.

Tension tests of the pipe were made on full-size specimens as well as on strips machined from the pipe. The average dimensions of the pipe, which was nominally a 1-inch pipe, were: Inner diameter, 1.05 inches; outer diameter, 1.325 inches. Eighteen-inch specimens from each end of three lengths of pipe were used. Snugly fitting plugs of mild steel were inserted in the ends of the pipe specimens to prevent them from collapsing in the jaws of the testing machine. The length of specimen between the jaws was about 10 inches. The results of these tests are given in Table 8 .

TABLE 6.-Tensile properties of wrought iron in the muck-bar state

$[A=$ iron made by Aston process; $\mathrm{HP}=$ hand-puddled iron; $F, M$, and $B=$ front, middle, and back of the muck-bar strip as rolled]

\begin{tabular}{|c|c|c|c|c|c|}
\hline \multirow{3}{*}{ Material } & \multicolumn{5}{|c|}{ Tensile properties } \\
\hline & \multirow{2}{*}{$\begin{array}{l}\text { Yield } \\
\text { point } 1\end{array}$} & \multirow{2}{*}{$\begin{array}{l}\text { Ultimate } \\
\text { tensile } \\
\text { strength }\end{array}$} & \multicolumn{2}{|c|}{ Elongation } & \multirow{2}{*}{$\begin{array}{l}\text { Reduc- } \\
\text { tion of } \\
\text { area }\end{array}$} \\
\hline & & & 2-inch & 8-inch & \\
\hline $\begin{array}{l}\text { AF4 } \\
\text { AF4 } \\
\text { AF4 }\end{array}$ & $\begin{array}{r}\text { Lbs./in. }{ }^{2} \\
21,300 \\
20,800 \\
20,000\end{array}$ & $\begin{array}{r}\text { I.bs./in. } .^{2} \\
32,800 \\
43,300 \\
38,500\end{array}$ & $\begin{array}{r}\text { Per cent } \\
12.5 \\
34.0 \\
18.5\end{array}$ & $\begin{array}{r}\text { Per cent } \\
6.5 \\
21.5 \\
11.5\end{array}$ & $\begin{array}{r}\text { Per cent } \\
12.5 \\
20.0 \\
15.5\end{array}$ \\
\hline $\begin{array}{l}\text { AM4 } \\
\text { AM4 } \\
\text { AM4 }\end{array}$ & $\begin{array}{l}20,850 \\
20,650 \\
20,000\end{array}$ & $\begin{array}{l}38,000 \\
41,350 \\
31,450\end{array}$ & $\begin{array}{l}17.5 \\
28.5 \\
(2)\end{array}$ & $\begin{array}{l}10.5 \\
18.5 \\
\left({ }^{2}\right)\end{array}$ & $\begin{array}{r}11.0 \\
19.5 \\
7.0\end{array}$ \\
\hline $\begin{array}{l}\mathrm{AB} 4-1-1 \\
\mathrm{AB} 4 \\
\mathrm{AB} 4\end{array}$ & $\begin{array}{l}21,800 \\
21,200 \\
21,250\end{array}$ & $\begin{array}{l}39,900 \\
42,250 \\
43,500\end{array}$ & $\begin{array}{l}25.0 \\
27.5 \\
27.5\end{array}$ & $\begin{array}{l}12.5 \\
20.0 \\
22.5\end{array}$ & $\begin{array}{l}18.0 \\
19.5 \\
26.5\end{array}$ \\
\hline Average.. & 20,870 & 39,000 & 23.9 & 15.4 & 16.4 \\
\hline $\begin{array}{l}\text { HPF1- } \\
\text { HPF1- }\end{array}$ & $\begin{array}{l}(3) \\
25,500\end{array}$ & $\begin{array}{r}{ }^{3} 13,600 \\
40,350\end{array}$ & 17.5 & 9.5 & 10.5 \\
\hline $\begin{array}{l}\text { HPM1 } \\
\text { HPM1- }\end{array}$ & $\begin{array}{l}24,300 \\
27,000\end{array}$ & $\begin{array}{l}34,000 \\
40,550\end{array}$ & $\begin{array}{l}(4) \\
12.5\end{array}$ & $\begin{array}{l}5.5 \\
6.5\end{array}$ & $\begin{array}{l}5.5 \\
9.0\end{array}$ \\
\hline 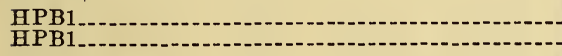 & $\begin{array}{l}29,400 \\
28,000\end{array}$ & $\begin{array}{l}42,400 \\
49,300\end{array}$ & $\begin{array}{l}13.5 \\
18.5\end{array}$ & $\begin{array}{r}7.0 \\
13.8\end{array}$ & $\begin{array}{l}10.0 \\
18.0\end{array}$ \\
\hline Average & 26,840 & 41,320 & 15.5 & 8.4 & 10.6 \\
\hline
\end{tabular}

1 From stress graph drawn by the Amsler machine.

2 Broke outside of gage marks.

3 Broke within the jaws of the machine at a weak spot.

4 Specimen too rough to show the gage marks. 
TABLE 7.-Tensile properties of wrought iron in the form of pipe skelp

$[\mathrm{A}=$ iron made by Aston process; $\mathrm{HP}=$ hand-puddled iron; $\mathrm{AHP}=$ composite iron, equal parts of $\mathrm{A}$ and $\mathrm{HP} ; \mathrm{F}, \mathrm{M}$, and $\mathrm{B}=$ front, middle, and back of the strip of skelp as rolled]

\begin{tabular}{|c|c|c|c|c|c|}
\hline \multirow{3}{*}{ Material } & \multicolumn{5}{|c|}{ Tensile properties } \\
\hline & \multirow{2}{*}{$\begin{array}{l}\text { Yield } \\
\text { point } 1\end{array}$} & \multirow{2}{*}{$\begin{array}{c}\text { Ultimate } \\
\text { tensile } \\
\text { strength }\end{array}$} & \multicolumn{2}{|c|}{ Elongation } & \multirow{2}{*}{$\begin{array}{l}\text { Reduc- } \\
\text { tion of } \\
\text { area }\end{array}$} \\
\hline & & & 2-inch & 8-inch & \\
\hline $\begin{array}{l}\text { AF1 } \\
\text { AM1 } 11 \\
\text { AM1 } \\
\text { AB1 } 1\end{array}$ & $\begin{array}{r}\text { LS./in.2 } \\
29,550 \\
33,100 \\
30,550 \\
31,000 \\
31,800 \\
30,550\end{array}$ & $\begin{array}{r}\text { Lbs./in. }{ }^{2} \\
44,100 \\
47,200 \\
44,950 \\
48,000 \\
47,200 \\
45,800\end{array}$ & $\begin{array}{r}\text { Per cent } \\
26.5 \\
30.0 \\
22.3 \\
27.5 \\
33.0 \\
23.0\end{array}$ & $\begin{array}{r}\text { Per cent } \\
21.0 \\
22.5 \\
18.0 \\
17.5 \\
24.5 \\
22.5\end{array}$ & $\begin{array}{r}\text { Per cent } \\
24.1 \\
23.7 \\
20.2 \\
22.5 \\
25.2 \\
21.6\end{array}$ \\
\hline $\begin{array}{l}\text { AF2 } \\
\text { AF2 } \\
\text { AM2 } \\
\text { AM2 } \\
\text { AB2 } \\
\text { AB2 }\end{array}$ & $\begin{array}{l}32,000 \\
28,800 \\
27,100 \\
29,700 \\
30,450 \\
32,500\end{array}$ & $\begin{array}{l}44,600 \\
44,400 \\
41,600 \\
44,550 \\
42,400 \\
45,000\end{array}$ & $\begin{array}{r}21.0 \\
20.0 \\
17.5 \\
(2) \\
16.0 \\
20.0\end{array}$ & $\begin{array}{r}17.0 \\
15.0 \\
12.0 \\
(2) \\
11.5 \\
16.0\end{array}$ & $\begin{array}{l}21.6 \\
16.6 \\
17.2 \\
(2) \\
15.2 \\
19.0\end{array}$ \\
\hline $\begin{array}{l}\text { A F3 } \\
\text { A F3 } \\
\text { AM3 } \\
\text { AM3 } \\
\text { AB3 } \\
\text { AB3 }\end{array}$ & $\begin{array}{l}29,850 \\
28,750 \\
28,600 \\
26,500 \\
31,900 \\
30,450\end{array}$ & $\begin{array}{l}44,500 \\
43,000 \\
44,200 \\
42,000 \\
45,000 \\
42,150\end{array}$ & $\begin{array}{l}19.0 \\
16.0 \\
20.0 \\
15.0 \\
20.0 \\
19.0\end{array}$ & $\begin{array}{l}16.5 \\
12.0 \\
14.0 \\
12.3 \\
1 \text { 1.. } \\
13.2\end{array}$ & $\begin{array}{l}16.4 \\
17.0 \\
16.7 \\
15.0 \\
19.5 \\
17.7\end{array}$ \\
\hline $\begin{array}{l}\text { AF4 } \\
\text { AF4 } \\
\text { AM4 } \\
\text { AM4 } \\
\text { AB4 }\end{array}$ & $\begin{array}{l}29,500 \\
32,650 \\
31,500 \\
28,300 \\
32,000 \\
32,500\end{array}$ & $\begin{array}{l}44,650 \\
46,900 \\
48,200 \\
44,550 \\
45,500 \\
48,200\end{array}$ & $\begin{array}{l}32.5 \\
20.0 \\
17.5 \\
21.0 \\
25.0 \\
22.5\end{array}$ & $\begin{array}{l}20.5 \\
12.5 \\
15.0 \\
16.0 \\
21.0 \\
14.5\end{array}$ & $\begin{array}{l}24.0 \\
17.8 \\
22.0 \\
18.5 \\
23.5 \\
203\end{array}$ \\
\hline A теragg & 30,400 & 44,940 & 22.8 & 16.3 & 19.7 \\
\hline $\begin{array}{l}\text { HPF1. } \\
\text { HPF1 } \\
\text { HPM1 } \\
\text { HPM1 } \\
\text { HPB1 } \\
\text { HPB1 }\end{array}$ & $\begin{array}{l}32,500 \\
34,000 \\
31,300 \\
32,000 \\
33,600 \\
36,100\end{array}$ & $\begin{array}{l}46,000 \\
45,150 \\
46,700 \\
47,250 \\
47,250 \\
46,750\end{array}$ & $\begin{array}{l}16.0 \\
13.0 \\
19.0 \\
17.0 \\
17.5 \\
17.0\end{array}$ & $\begin{array}{r}11.0 \\
9.5 \\
13.0 \\
13.5 \\
12.5 \\
12.0\end{array}$ & $\begin{array}{l}18.0 \\
13.7 \\
16.6 \\
17.0 \\
16.0 \\
17.6\end{array}$ \\
\hline $\begin{array}{l}\text { HPF2 } \\
\text { HPF2 } \\
\text { HPM2 } \\
\text { HPM2 } \\
\text { HPB2 } \\
\text { HPB2 }\end{array}$ & $\begin{array}{l}32,350 \\
34,000 \\
31,500 \\
32,350 \\
36,500 \\
36,450\end{array}$ & $\begin{array}{l}46,550 \\
44,350 \\
40,500 \\
42,800 \\
46,250 \\
47,800\end{array}$ & $\begin{array}{r}20.0 \\
12.0 \\
10.0 \\
(3) \\
15.0 \\
17.5\end{array}$ & $\begin{array}{r}15.0 \\
9.0 \\
6.2 \\
(3) \\
10.0 \\
14.5\end{array}$ & $\begin{array}{r}18.1 \\
14.7 \\
11.5 \\
9.9 \\
12.0 \\
15.2\end{array}$ \\
\hline $\begin{array}{l}\text { HPF3 } \\
\text { HPF3 } \\
\text { HPM3 } \\
\text { HPM3 } \\
\text { HPB3 } \\
\text { HPB3 }\end{array}$ & $\begin{array}{l}33,000 \\
34,350 \\
32,050 \\
32,000 \\
33,850 \\
34,000\end{array}$ & $\begin{array}{l}42,000 \\
45,950 \\
42,400 \\
45,550 \\
43,450 \\
43,000\end{array}$ & $\begin{array}{l}11.5 \\
18.0 \\
(3) \\
13.5 \\
12.5 \\
14.0\end{array}$ & $\begin{array}{r}7.5 \\
13.0 \\
(3) \\
10.2 \\
9.0 \\
8.0\end{array}$ & $\begin{array}{r}11.5 \\
17.1 \\
15.5 \\
9.5 \\
14.5\end{array}$ \\
\hline $\begin{array}{l}\text { HPF4 } \\
\text { HPF4 } \\
\text { HPM4 } \\
\text { HPM4 } \\
\text { HPB4 } \\
\text { HPB4 }\end{array}$ & $\begin{array}{l}34,500 \\
34,150 \\
34,850 \\
33,000 \\
33,850 \\
36,300\end{array}$ & $\begin{array}{l}43,500 \\
42,300 \\
45,450 \\
41,100 \\
45,000 \\
46,450\end{array}$ & $\begin{array}{r}11.0 \\
11.0 \\
10.0 \\
7.5 \\
12.5 \\
12.0\end{array}$ & $\begin{array}{l}5.5 \\
8.0 \\
6.0 \\
4.5 \\
9.5 \\
6.2\end{array}$ & $\begin{array}{r}10.5 \\
15.5 \\
9.1 \\
7.1 \\
13.5 \\
8.1\end{array}$ \\
\hline A verage & 33,690 & 44,730 & 14.0 & 9.8 & 13.5 \\
\hline 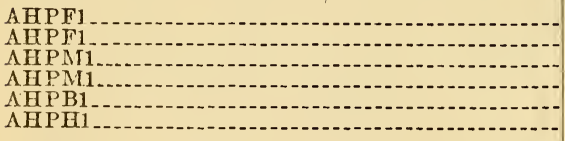 & $\begin{array}{l}32,150 \\
31,750 \\
31,500 \\
31,950 \\
32,850 \\
31,750\end{array}$ & $\begin{array}{l}41,100 \\
42,500 \\
40,500 \\
43,100 \\
42,800 \\
40,000\end{array}$ & $\begin{array}{r}8.5 \\
12.0 \\
(3) \\
12.5 \\
12.5 \\
10.5\end{array}$ & $\begin{array}{r}6.0 \\
8.0 \\
(3) \\
9.0 \\
9.0 \\
6.5\end{array}$ & $\begin{array}{r}10.5 \\
13.0 \\
13.0 \\
12.5 \\
11.5 \\
9.5\end{array}$ \\
\hline $\begin{array}{l}\text { AHPF2 } \\
\text { AHPF2 } \\
\text { AHPM2 } \\
\text { AHPM2 } \\
\text { AHPB2 } \\
\text { AHPB2 }\end{array}$ & $\begin{array}{l}31,000 \\
30,300 \\
30,050 \\
28,850 \\
34,150 \\
31,250\end{array}$ & $\begin{array}{l}38,000 \\
31,300 \\
39,400 \\
36,050 \\
43,550 \\
40,750\end{array}$ & $\begin{array}{r}9.0 \\
6.5 \\
11.0 \\
9.0 \\
18.5 \\
12.0\end{array}$ & $\begin{array}{r}5.5 \\
2.0 \\
7.0 \\
4.5 \\
14.0 \\
7.5\end{array}$ & $\begin{array}{r}10.0 \\
9.5 \\
13.3 \\
11.5 \\
16.3 \\
13.5\end{array}$ \\
\hline
\end{tabular}


TABLE 7.-Tensile properties of wrought iron in the form of pipe skelp-Continued

$[\mathrm{A}=$ iron made by $\mathrm{Aston}$ process; $\mathrm{HP}=$ hand-puddled iron; $\mathrm{AHP}=$ composite iron, equal parts of $\mathrm{A}$ and $\mathrm{HP} ; \mathrm{F}, \mathrm{M}$, and $\mathrm{B}=$ front, middle, and back of the strip of skelp as rolled]

\begin{tabular}{|c|c|c|c|c|c|}
\hline \multirow{3}{*}{ Material } & \multicolumn{5}{|c|}{ Tensile properties } \\
\hline & \multirow{2}{*}{$\begin{array}{l}\text { Yield } \\
\text { point }\end{array}$} & \multirow{2}{*}{$\begin{array}{l}\text { Ultimate } \\
\text { tensile } \\
\text { strength }\end{array}$} & \multicolumn{2}{|c|}{ Elongation } & \multirow{2}{*}{$\begin{array}{l}\text { Reduc- } \\
\text { tion of } \\
\text { area }\end{array}$} \\
\hline & & & 2-inch & 8-inch & \\
\hline $\begin{array}{l}\text { AHPF3 } \\
\text { AHPF3... } \\
\text { AHPM3 } \\
\text { AHPM3 } \\
\text { AHPB3 } \\
\text { AHPB3... }\end{array}$ & $\begin{array}{r}\text { Lbs./in. }{ }^{2} \\
33,600 \\
32,800 \\
30,500 \\
31,600 \\
31,500 \\
32,400\end{array}$ & $\begin{array}{r}\text { Lbs./in. }{ }^{2} \\
44,150 \\
44,600 \\
43,000 \\
43,050 \\
43,000 \\
46,150\end{array}$ & $\begin{array}{r}\text { Per cent } \\
(2) \\
15.0 \\
13.5 \\
12.0 \\
13.0 \\
18.0\end{array}$ & $\begin{array}{r}\text { Per cent } \\
(2) \\
11.0 \\
10.5 \\
8.0 \\
9.5 \\
14.0\end{array}$ & $\begin{array}{r}\text { Per cent } \\
(2) \\
14.5 \\
14.0 \\
14.0 \\
14.5 \\
15.3\end{array}$ \\
\hline $\begin{array}{l}\text { AHPF4 } \\
\text { AHPF4 } \\
\text { AHPM4 } 4 \text { AHPM4 } \\
\text { AHPMB } \\
\text { AHPB } \\
\text { AHPB4 }\end{array}$ & $\begin{array}{l}31,250 \\
33,650 \\
29,500 \\
34,250 \\
33,400 \\
32,000\end{array}$ & $\begin{array}{l}42,000 \\
41,700 \\
37,500 \\
44,250 \\
42,700 \\
44,350\end{array}$ & $\begin{array}{r}11.0 \\
8.5 \\
6.0 \\
9.0 \\
\text { (3) } \\
15.0\end{array}$ & $\begin{array}{r}7.5 \\
4.5 \\
4.5 \\
5.0 \\
\quad 3)^{3} \\
9.0\end{array}$ & $\begin{array}{r}10.0 \\
9.0 \\
10.0 \\
9.5 \\
10.5 \\
14.5\end{array}$ \\
\hline A verage... & 31,830 & 41,480 & 12.6 & 7.7 & 12.6 \\
\hline
\end{tabular}

2 Broke in flllet.

Broke outside of gage points.

TABLE 8.-Tensile properties of 1-inch wrought-iron pipe, as determined on full-size specimens

[ $A=$ iron made by Aston process; $\mathrm{H} \mathrm{P}=$ hand-puddled iron; $\mathrm{AHP}=$ composite iron containing equal amounts of $A$ and $H P ; x$ and $\bar{y}$ indicate the two ends of the pipe length from which specimens were cut]

\begin{tabular}{|c|c|c|c|}
\hline \multirow{2}{*}{ Designation of material } & \multicolumn{3}{|c|}{ Tensile properties 1} \\
\hline & $\begin{array}{l}\text { Yield } \\
\text { point }\end{array}$ & $\begin{array}{c}\text { Breaking } \\
\text { load }\end{array}$ & $\begin{array}{l}\text { Elonga- } \\
\text { tion } \\
\text { (8-inch) }\end{array}$ \\
\hline 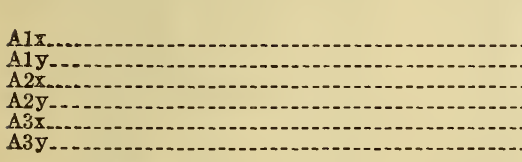 & $\begin{array}{r}\text { Pounds } \\
17,050 \\
17,200 \\
17,400 \\
17,700 \\
17,400\end{array}$ & $\begin{array}{r}\text { Pounds } \\
25,100 \\
24,600 \\
24,850 \\
25,000 \\
24,150 \\
24,550\end{array}$ & $\begin{array}{r}\text { Per cent } \\
17.0 \\
18.0 \\
13.0 \\
16.0 \\
11.5 \\
13.0\end{array}$ \\
\hline Average........... & 17,350 & 24,700 & 14.7 \\
\hline 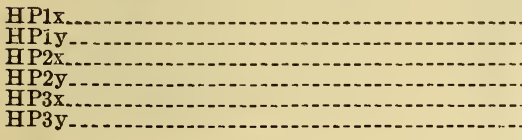 & $\begin{array}{r}19,000 \\
18,700 \\
19,900 \\
19,650\end{array}$ & $\begin{array}{l}26,900 \\
25,700 \\
27,300 \\
26,850 \\
27,550 \\
27,700\end{array}$ & $\begin{array}{r}12.0 \\
8.0 \\
13.0 \\
13.0 \\
13.0 \\
12.5\end{array}$ \\
\hline Average.......... & 19,300 & 27,000 & 11.9 \\
\hline 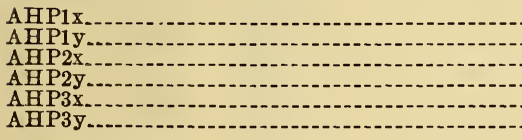 & $\begin{array}{r}19,000 \\
18,750 \\
18,300\end{array}$ & $\begin{array}{l}25,650 \\
24,550 \\
25,400 \\
25,700 \\
25,750 \\
25,000\end{array}$ & $\begin{array}{r}9.5 \\
8.5 \\
9.0 \\
8.5 \\
10.5 \\
\left({ }^{2}\right)\end{array}$ \\
\hline \multirow[t]{3}{*}{ Average. } & 18,700 & 25,350 & 9.2 \\
\hline & & \multicolumn{2}{|c|}{$\begin{array}{l}\text { Average tensile } \\
\text { properties } 2\end{array}$} \\
\hline & & $\begin{array}{l}\text { Yield } \\
\text { point }\end{array}$ & $\begin{array}{l}\text { Ultimate } \\
\text { tensile } \\
\text { strength }\end{array}$ \\
\hline 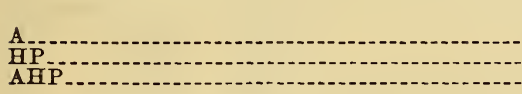 & & $\begin{array}{r}\text { Lbs./in } \\
33,150 \\
36,900 \\
35,700\end{array}$ & $\begin{array}{r}\text { Lbs./in. } \\
47,250 \\
51,600 \\
48,450\end{array}$ \\
\hline
\end{tabular}

1 A Riehle testing machine 100,000-pound capacity was used; yield point was determined by drop of beam.

2 Broke outside of gage marks 
In many specifications for pipe the tensile properties may be determined on strips cut from the pipe instead of on full-size pipe specimens. Although it is usually intended that the strip method shall apply only to pipe of relatively large diameter, it is sometimes used for pipe of small diameter. It was considered of interest, therefore, to determine the tensile properties of the three types of pipe on longitudinal strips machined from the pipe. Eighteen-inch specimens were machined without any flattening of the pipe, so that the edges were parallel (not radial). The width in the shoulders was 2 inches and in the central portion, over a length of 10 inches, the width was 1 inch. The specimens were tested in an Amsler machine, special grips being used so as to maintain the original curvature throughout the test. It will be noted, by comparing the results (Table 9) with those in Table 8 , that the most marked effect produced by using specimens of this form was the lowering of the elongation.

(2) Torsional Properties.-Torsion tests were made on four specimens of each of the three kinds of pipe. The specimens for the tests, which were carried out in a Riehle torsion testing machine of 60,000 -pounds capacity, were 44 inches in length and the ends were plugged as in the tension tests of pipe. The length of the specimen between grips was 38 inches. The results obtained are summarized in Table 10 and the appearance of the pipe after testing is shown in Figure 8.

TABLE 9.-Tensile properties of 1-inch wrought-iron pipe determined on longitudinal strips machined from the pipe

[ $\mathrm{A}=$ iron made by Aston process; $\mathrm{H} \mathrm{P}=$ hand-puddled iron; $\mathrm{AH}=$ composite iron containing equal parts of $\mathrm{A}$ and $\mathrm{HP}]$

\begin{tabular}{|c|c|c|c|}
\hline \multirow[b]{2}{*}{ Material } & \multirow[b]{2}{*}{$\begin{array}{l}\text { Yield } \\
\text { point }\end{array}$} & \multicolumn{2}{|c|}{ Tensile properties } \\
\hline & & $\begin{array}{c}\text { Ultimate } \\
\text { tensile } \\
\text { strength }\end{array}$ & $\begin{array}{c}\text { Elonga- } \\
\text { tion (8- } \\
\text { inch) }\end{array}$ \\
\hline A. & $\begin{array}{l}\text { Lbs./in. }{ }^{2} \\
36,300 \\
33,400 \\
36,650 \\
33,700 \\
35,700 \\
35,150\end{array}$ & \begin{tabular}{|r|} 
Lbs./in. ${ }^{2}$ \\
49,200 \\
47,350 \\
51,750 \\
48,250 \\
50,600 \\
49,500
\end{tabular} & $\begin{array}{r}\text { Per cent } \\
9.0 \\
8.0 \\
12.0 \\
9.6 \\
11.5 \\
10.0\end{array}$ \\
\hline Average.............. & 35,150 & 49,440 & 10.0 \\
\hline HP...... & $\begin{array}{l}37,500 \\
35,900 \\
38,500 \\
45,750 \\
39,850 \\
40,750\end{array}$ & $\begin{array}{l}52,250 \\
51,150 \\
52,050 \\
52,800 \\
56,100 \\
55,100\end{array}$ & $\begin{array}{r}8.8 \\
9.8 \\
7.0 \\
7.5 \\
13.1 \\
9.9\end{array}$ \\
\hline Average. & 39,700 & 53,240 & 8.9 \\
\hline AHP & $\begin{array}{r}36,100 \\
36,150 \\
38,100 \\
34,100 \\
38,950 \\
37,400\end{array}$ & $\begin{array}{l}50,300 \\
49,900 \\
51,850 \\
48,750 \\
49,900 \\
51,350\end{array}$ & $\begin{array}{l}8.0 \\
5.7 \\
7.0 \\
9.5 \\
5.0 \\
6.8\end{array}$ \\
\hline A verage & 36,800 & 50,340 & 7.0 \\
\hline
\end{tabular}


B. S. Journal of Research, RP124

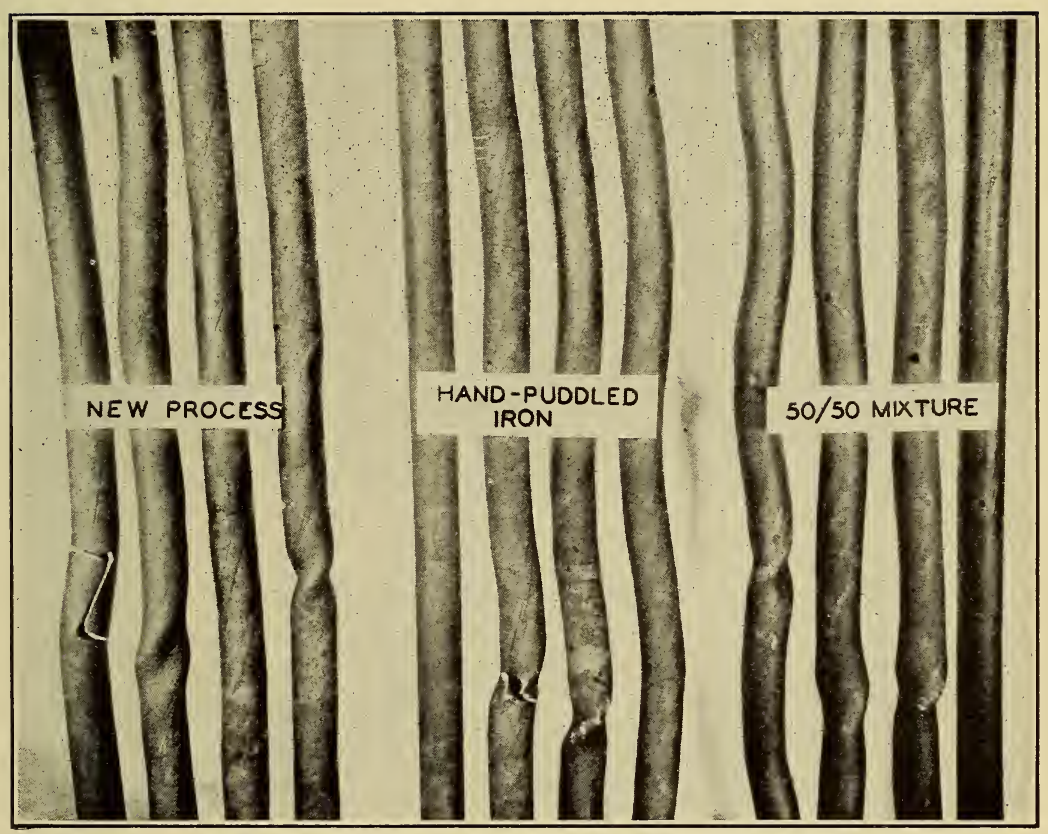

FIgURE 8.-Appearance of the pipe after the torsion test 
TABLE 10.-Torsional properties of 1-inch wrought-iron pipe

[ $\mathrm{A}=$ iron made by Aston process; $\mathrm{H} \mathrm{P}=$ hand-puddled iron; $\mathrm{AH} \mathrm{P}=$ composite iron containing equal parts $A$ and $\mathrm{HP}$ ]

\begin{tabular}{|c|c|c|c|c|}
\hline \multirow{2}{*}{ Material } & \multicolumn{4}{|c|}{ Torsional properties } \\
\hline & \multirow{2}{*}{$\begin{array}{c}\text { Yield } \\
\text { point } 1\end{array}$} & \multirow{2}{*}{$\begin{array}{c}\begin{array}{c}\text { Maxi- } \\
\text { mum } \\
\text { moment }\end{array} \\
\text { In./lbs. } \\
9,850 \\
10,700 \\
10,300 \\
10,750\end{array}$} & \multicolumn{2}{|c|}{$\begin{array}{c}\text { Total number of } \\
360^{\circ} \text { twists }\end{array}$} \\
\hline 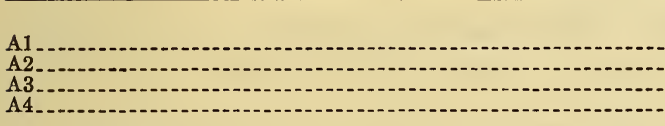 & & & $\begin{array}{l}\circ \\
1,260 \\
1,465 \\
1,340 \\
1,380\end{array}$ & $\begin{array}{l}\text { Turns } \\
\text { 3. } 5 \\
4.07 \\
3.72 \\
3.83\end{array}$ \\
\hline A verage & 5,975 & 10,395 & 1,361 & 3.78 \\
\hline HP2 & $\begin{array}{l}6,550 \\
7,000 \\
6,800 \\
6,850\end{array}$ & $\begin{array}{r}11,250 \\
9,600 \\
11,650 \\
11,450\end{array}$ & $\begin{array}{r}755 \\
380 \\
1,170 \\
1,180\end{array}$ & $\begin{array}{l}2.09 \\
1.05 \\
3.25 \\
3.27\end{array}$ \\
\hline Average & 6,800 & 10,985 & 871 & 2.42 \\
\hline $\begin{array}{l}\text { AH } \\
\text { AHP2 } \\
\text { AH }{ }^{2}{ }^{2}{ }^{2} \\
\text { AH }\end{array}$ & $\begin{array}{l}5,000 \\
6,000 \\
5,600 \\
5,650\end{array}$ & $\begin{array}{r}10,450 \\
11,100 \\
9,850 \\
10,750\end{array}$ & $\begin{array}{r}1,260 \\
1,450 \\
810 \\
1,500\end{array}$ & $\begin{array}{l}3.50 \\
4.03 \\
2.25 \\
4.16\end{array}$ \\
\hline Average & 5,560 & 10,540 & 1,255 & 3.48 \\
\hline
\end{tabular}

1 By drop of beam.

2 The weld parted at the load end during the test.

(3) Flattening Tests.-Six specimens, each 6 inches long, of each of the three kinds of wrought-iron pipe were used in the flattening tests. The load was applied perpendicularly along the entire length of the specimen and at $90^{\circ}$ to the weld. Table 11 contains the results of the tests.

\section{TABLE 11.-Results of flattening tests of 1-inch wrought-iron pipe}

[ $\mathrm{A}=$ iron made by Aston process; $\mathrm{H} \mathrm{P}=$ hand-puddled iron; $\mathrm{AHP}=$ composite iron containing equal parts of $\mathrm{A}$ and $\mathrm{HP}$ ]

\begin{tabular}{r|r|r}
\hline Material & $\begin{array}{r}\text { Maximum } \\
\text { load }\end{array}$ & Remarks \\
\hline & $\begin{array}{r}\text { Pounds } \\
9,150\end{array}$ & All fractures were fibrous, no defective welds revealed. \\
& 9,150 & \\
A1 & 9,900 \\
A1 & 8,200 \\
A2 & 9,100 \\
A2 Not across the weld. \\
A33
\end{tabular}


(b) 1-INCH ROUNDS

The 1-inch rounds used were not available as a commercial product and were made especially for this study. In addition to tension and torsion tests which were also made on the pipe materials, several other mechanical tests which can not be carried out readily on pipe or on flat strips were made.

(1) Tensile Properties.-The tensile properties of the wrought iron made by the new process in the form of 1-inch rounds were

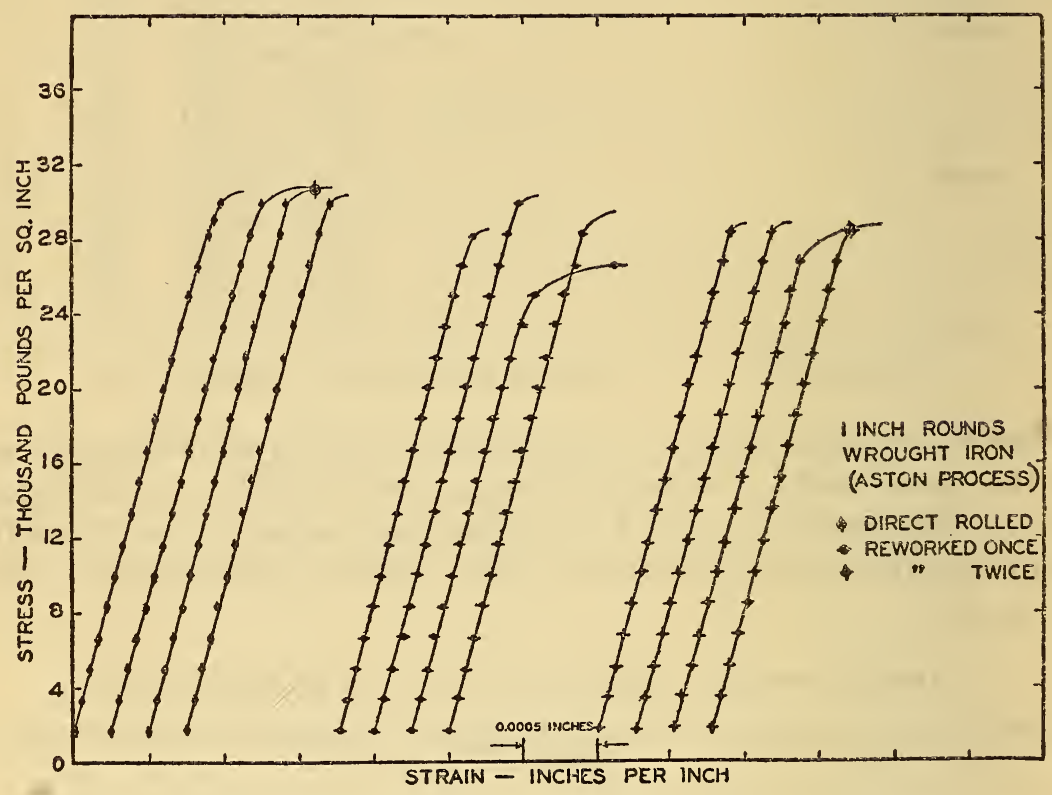

FIgURE 9.-Stress-strain curves of wrought iron made by the Aston process in 1-inch rounds tested in tension

determined on both the full-size bar and on specimens machined from the bar. The results are summarized in Table 12. In the case of the machined specimens, strain measurements were made by means of the Ewing extensometer and the curves summarizing these results are given in Figure 9. It is very evident from the results obtained that the tensile properties of the iron were only slightly changed by the "refining" given the material before rolling into the form of 1 -inch rounds. The tensile strength was lowered slightly and the elongation increased somewhat as the amount of working given the material was increased. 
TABLE 12.-Tensile properties of wrought iron made by the Aston process rolled into the form of 1 -inch rounds

TESTS MADE ON FULL-SIZE BARS, 18 INCHES LONG

\begin{tabular}{|c|c|c|c|c|c|c|}
\hline \multirow{3}{*}{ Specimen } & \multirow{3}{*}{ Material } & \multicolumn{5}{|c|}{ Tensile properties } \\
\hline & & \multirow{2}{*}{$\begin{array}{l}\text { Yield } \\
\text { point }\end{array}$} & \multirow{2}{*}{$\begin{array}{l}\text { Ultimate } \\
\text { tensile } \\
\text { strength }\end{array}$} & \multicolumn{2}{|c|}{ Elongation } & \multirow{2}{*}{$\begin{array}{l}\text { Reduc- } \\
\text { tion of } \\
\text { area }\end{array}$} \\
\hline & & & & 2-inch & 8-inch & \\
\hline $\begin{array}{l}1 \mathrm{aa} . . \\
1 \mathrm{bb} . \\
1 \mathrm{cc}- \\
\text { idd. }\end{array}$ & \multirow[t]{2}{*}{$\begin{array}{l}\text { Rolled directly from billet to } 1 \text {-inch } \\
\text { round }\end{array}$} & $\begin{array}{r}\text { Lbs./in. }{ }^{2} \\
27,750 \\
28,600 \\
28,700 \\
29,300\end{array}$ & $\begin{array}{r}\text { Lbs./in. }{ }^{2} \\
49,050 \\
48,650 \\
49,250 \\
50,000\end{array}$ & $\begin{array}{r}\text { Per cent } \\
40.0 \\
(2) \\
44.0\end{array}$ & $\begin{array}{r}\text { Per cent } \\
27.5 \\
25.0 \\
(2) \\
27.5\end{array}$ & $\begin{array}{r}\text { Per cent } \\
47.4 \\
48.1 \\
\left({ }^{2}\right) \\
45.3\end{array}$ \\
\hline \multirow{2}{*}{ 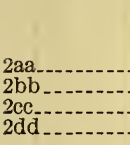 } & & 28,590 & 49,240 & 42.0 & 26.7 & 46. 9 \\
\hline & "Single refined"- & $\begin{array}{l}28,150 \\
28,550 \\
28,550 \\
28,550\end{array}$ & $\begin{array}{l}48,850 \\
48,650 \\
49,150 \\
49,000\end{array}$ & $\begin{array}{l}47.0 \\
49.0 \\
49.0 \\
47.0\end{array}$ & $\begin{array}{l}30.0 \\
31.5 \\
29.3 \\
30.0\end{array}$ & $\begin{array}{l}50.7 \\
50.4 \\
50.9 \\
49.5\end{array}$ \\
\hline \multirow{3}{*}{$\begin{array}{l}3 \mathrm{aa} \\
3 \mathrm{bb} \\
3 \mathrm{bcc} \\
3 \mathrm{cc}-\ldots \\
3 \mathrm{dd}\end{array}$} & \multirow{3}{*}{ "Double refined". } & 28,450 & 48,910 & 48.0 & 30.2 & 50.4 \\
\hline & & $\begin{array}{l}27,300 \\
27,650 \\
28,100 \\
28,800\end{array}$ & $\begin{array}{l}48,050 \\
47,900 \\
48,400 \\
48,200\end{array}$ & $\begin{array}{l}45.0 \\
50.0 \\
49.5 \\
(3)\end{array}$ & $\begin{array}{l}29.5 \\
31.5 \\
30.3 \\
(3)\end{array}$ & $\begin{array}{l}51.9 \\
51.5 \\
50.7 \\
53.1\end{array}$ \\
\hline & & 27,960 & 48,140 & 48.2 & 30.4 & 51.8 \\
\hline
\end{tabular}

TESTS MADE ON BARS MACHINED TO 0.875-INCH DIAMETER, 8-INCH GAGE LENGTH

\begin{tabular}{|c|c|c|c|c|c|c|}
\hline \multirow[t]{2}{*}{$\begin{array}{l}1 \mathrm{a} \\
1 \mathrm{~b} \\
1 \mathrm{c}-\ldots \\
1 \mathrm{~d}-\ldots\end{array}$} & $\begin{array}{l}\text { Rolled directly from billet to } 1 \text {-inch } \\
\text { round }\end{array}$ & $\begin{array}{l}29,850 \\
30,500 \\
30,700 \\
29,850\end{array}$ & $\begin{array}{l}49,750 \\
50,250 \\
50,250 \\
49,950\end{array}$ & $\begin{array}{l}44.0 \\
43.5 \\
44.0 \\
43.0\end{array}$ & $\begin{array}{l}28.5 \\
29.0 \\
27.5 \\
30.0\end{array}$ & $\begin{array}{l}47.3 \\
47.9 \\
46.6 \\
48.1\end{array}$ \\
\hline & Average..... & 30,220 & 50,050 & 43.6 & 28.7 & 47.5 \\
\hline \multirow[t]{2}{*}{$\begin{array}{l}2 \mathrm{a} \\
2 \mathrm{~b} \\
2 \mathrm{c} \\
2 \mathrm{~d}\end{array}$} & "Single refined" .. & $\begin{array}{l}28,200 \\
29,850 \\
28,200 \\
28,200\end{array}$ & $\begin{array}{l}48,550 \\
48,150 \\
47,900 \\
48,100\end{array}$ & $\begin{array}{l}46.0 \\
46.0 \\
45.0 \\
46.0\end{array}$ & $\begin{array}{l}30.5 \\
30.5 \\
29.0 \\
30.0\end{array}$ & $\begin{array}{l}50.0 \\
49.2 \\
50.7 \\
49.8\end{array}$ \\
\hline & Average...... & 28,610 & 48,175 & 45.7 & 30.0 & 50.0 \\
\hline \multirow[t]{2}{*}{$\begin{array}{l}3 \mathrm{a}-\ldots \\
3 \mathrm{~b} \\
3 \mathrm{c} \\
3 \mathrm{~d}\end{array}$} & "Double refined"... & $\begin{array}{l}28,700 \\
29,000 \\
28,700 \\
28,200\end{array}$ & $\begin{array}{l}48,450 \\
48,350 \\
48,500 \\
47,750\end{array}$ & $\begin{array}{r}49.0 \\
43.5 \\
45.0 \\
46.0\end{array}$ & $\begin{array}{l}29.5 \\
28.5 \\
29.0 \\
29.5\end{array}$ & $\begin{array}{l}51.1 \\
46.9 \\
48.3 \\
50.3\end{array}$ \\
\hline & A verage-- & 28,650 & 48,260 & 45.9 & 29.1 & 49.1 \\
\hline
\end{tabular}

1 By stress graph drawn by the Amsler machine.

2 Broke in jaws of the machine, outside of gage marks.

3 Broke outside of gage marks.

(2) Torsional Properties.-Torsion tests of the 1-inch round material were carried out on specimens 15 inches long, the central portion of which had been machined to a diameter of one-half inch over a 10-inch gage length. The results are given in Table 13, and in Figure 10 are given curves showing the behavior of the iron when stressed in torsion. It will be seen that there was no pronounced change in the torsional properties produced by the refining given the iron. This observation is in agreement with the results of the tension tests.

(3) Impact Resistance.-The resistance to impact of the iron in the form of notched specimens (notched-bar impact resistance) was determined by two methods, viz, resistance to repeated blows (Eden- 
Foster test) and resistance to a single blow (Izod test). The results (Tables 14 and 15), in general, indicate no marked difference in the notched-bar single-blow impact resistance resulting from the extra mechanical working which some of the material received. In the case of the repeated-blow tests, however, the average impact resist-

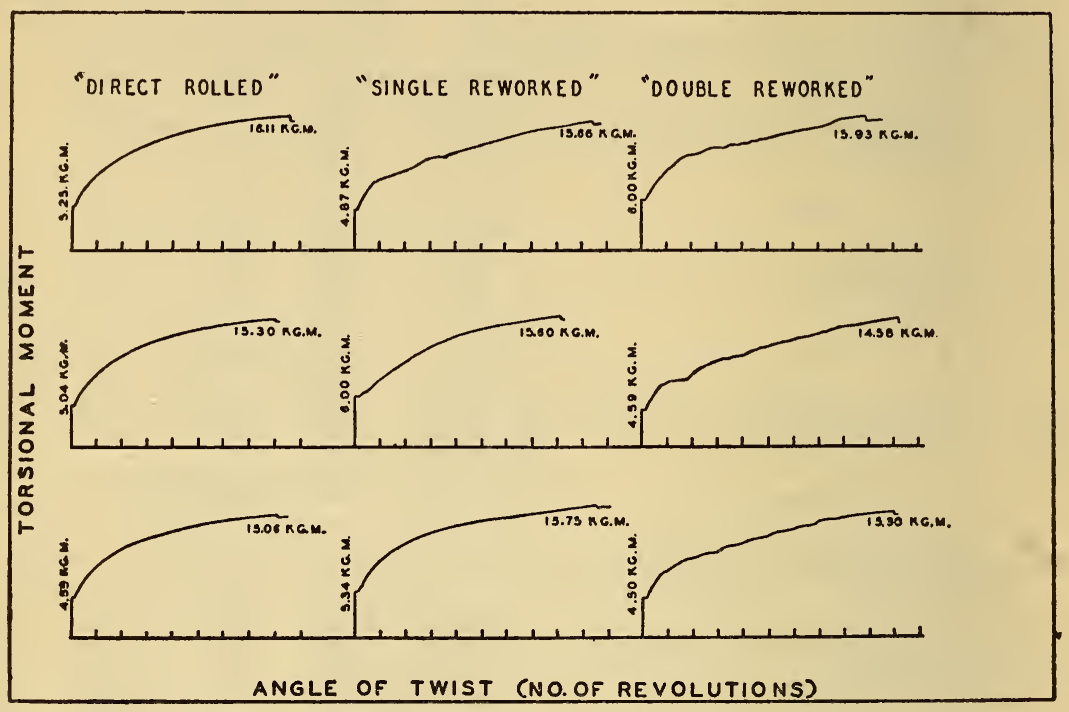

FIGURE 10.-Stress-strain curves of wrought iron made by the Aston process in 1-inch rounds tested in torsion

ance was somewhat improved by the double refining given to some of the iron. In all cases the conventional test specimens were machined out of the 1-inch round, the full-size bar was not used.

TABLE 13.-Torsional properties of wrought iron made by the Aston process rolled into the form of 1-inch rounds

\begin{tabular}{|c|c|c|c|c|}
\hline \multirow[b]{2}{*}{$\begin{array}{l}\text { Specimen } \\
\text { No. }\end{array}$} & \multirow[b]{2}{*}{ Material } & \multicolumn{3}{|c|}{ Torsional properties } \\
\hline & & $\begin{array}{l}\text { Yield } \\
\text { point }\end{array}$ & $\underset{\text { moment }}{\text { Maximum }}$ & $\begin{array}{l}\text { Twist } \\
\text { (number } \\
\text { of } 360^{\circ} \\
\text { twists) }\end{array}$ \\
\hline \multirow[t]{2}{*}{ 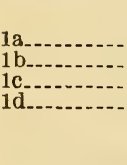 } & \multirow[t]{2}{*}{ Rolled directly from billet to 1 -inch round... } & $\begin{array}{r}\text { Ft./lbs. } \\
39.0 \\
33.0 \\
38.0 \\
36.5\end{array}$ & $\begin{array}{r}\text { Ft. } . / \text { lbs. } \\
119.5 \\
109.0 \\
116.5 \\
110.5\end{array}$ & $\begin{array}{l}9.64 \\
8.30 \\
8.80 \\
8.13\end{array}$ \\
\hline & & 36.6 & 114.0 & 8.72 \\
\hline \multirow[t]{2}{*}{ 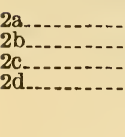 } & \multirow{2}{*}{ 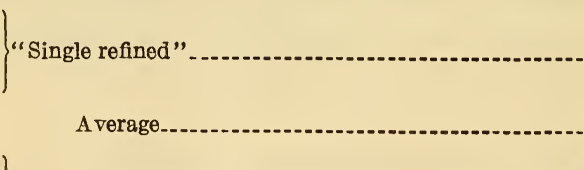 } & $\begin{array}{l}47.5 \\
38.5 \\
35.0 \\
33.0\end{array}$ & $\begin{array}{l}120.0 \\
114.0 \\
113.5 \\
105.5\end{array}$ & $\begin{array}{r}10.34 \\
9.60 \\
9.50 \\
10.33\end{array}$ \\
\hline & & 38.5 & 113.2 & 9.95 \\
\hline \multirow[t]{2}{*}{ 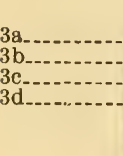 } & \multirow{2}{*}{$\int_{\text {" Double refined" }}$ A verage } & $\begin{array}{l}40.0 \\
32.5 \\
43.5 \\
33.0\end{array}$ & $\begin{array}{l}110.5 \\
113.0 \\
105.5\end{array}$ & $\begin{array}{r}10.15 \\
8.35 \\
10.35\end{array}$ \\
\hline & & 37.2 & 109.7 & 9.61 \\
\hline
\end{tabular}


TABLE 14.-Repeated-blow notched-bar impact resistance of wrought iron made by the Aston process rolled to the form of 1 -inch rounds

[Eden-Foster test. Specimens were $61 / 2$ inches long and one-half inch diameter, with a round flleted notch 0.05 inch deep. A 2-pound hammer with a 4-inch drop was used]

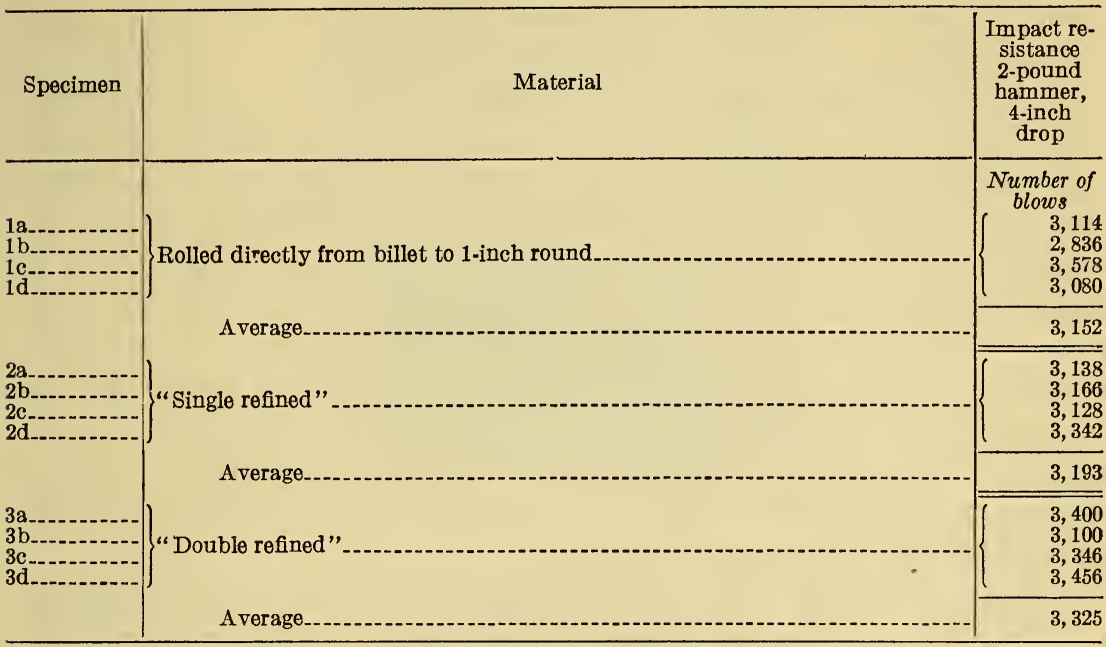

TABLE 15.-Single-blow notched-bar impact resistance of wrought iron made bu the Aston process rolled to the form of 1-inch rounds

[Izod test. Triple-notch specimens $\left(1 \mathrm{~cm}^{2}\left(0.394\right.\right.$ by 0.394 inch), section, $45^{\circ} \mathrm{V}$ notch, 0.079 inch deep) were used, the notches being arranged $90^{\circ}$ apart on three sides of the specimen. Capacity of Izod pendulum machine $120 \mathrm{ft} . / \mathrm{lbs}$.

\begin{tabular}{|c|c|c|c|c|}
\hline \multirow{2}{*}{ Specimen } & \multirow{2}{*}{ Material } & \multicolumn{3}{|c|}{$\begin{array}{c}\text { Impact resistance energy } \\
\text { absorbed } 1\end{array}$} \\
\hline & & a & $\mathrm{b}$ & $\mathrm{c}^{2}$ \\
\hline \multirow[t]{2}{*}{$\begin{array}{l}1 \mathrm{a} \\
1 \mathrm{~b} \\
1 \mathrm{c} \\
1 \mathrm{~d} \\
1 \mathrm{e} \\
1 \mathrm{f} \\
1 \mathrm{~g} \\
1 \mathrm{~h}\end{array}$} & \multirow[t]{2}{*}{ Rolled directly from billet to 1 -inch round... } & $\begin{array}{l}51.5 \\
55 \\
53 \\
50 \\
57 \\
55 \\
50 \\
61\end{array}$ & $\begin{array}{c}\text { Ft. } . l b s . \\
44.5 \\
52 \\
57 \\
53 \\
53 \\
56 \\
50 \\
54.5\end{array}$ & $\begin{array}{l}57 \\
51.5 \\
46 \\
51 \\
52 \\
47 \\
57 \\
57\end{array}$ \\
\hline & & - & -....-. & 52.6 \\
\hline \multirow[t]{2}{*}{ 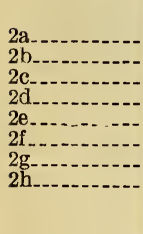 } & "Single refined"... & $\begin{array}{l}52 \\
55.5 \\
51 \\
50 \\
43 \\
50 \\
50 \\
50\end{array}$ & $\begin{array}{l}52 \\
55 \\
52 \\
55 \\
42 \\
51 \\
55 \\
47\end{array}$ & $\begin{array}{l}51 \\
52 \\
50 \\
45 \\
45 \\
50 \\
50 \\
45\end{array}$ \\
\hline & \multirow{3}{*}{ "Double refined"'-. } & 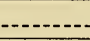 & - & 49.6 \\
\hline \multirow[t]{2}{*}{$\begin{array}{l}3 \mathrm{a} \\
3 \mathrm{~b} \\
3 \mathrm{c} \\
3 \mathrm{~d} \\
3 \mathrm{e} \\
3 \mathrm{f} \\
3 \mathrm{~g} \\
3 \mathrm{~h}\end{array}$} & & $\begin{array}{l}55 \\
53 \\
50.5 \\
56.5 \\
48 \\
52 \\
53 \\
56\end{array}$ & $\begin{array}{l}-53 \\
43 \\
58 \\
46 \\
52.5 \\
55 \\
55\end{array}$ & $\begin{array}{l}-53 \\
50 \\
48 \\
51.5 \\
49 \\
45.5 \\
44.5\end{array}$ \\
\hline & & & & 51.3 \\
\hline
\end{tabular}

\footnotetext{
1 None of the specimens fractured completely upon impact.
}

${ }_{2} \mathrm{a}, \mathrm{b}$, and $\mathrm{c}$ indicate the three notches on each specimen. 


\section{CORROSION RESISTANCE}

\section{(a) LABORATORY CORROSION TEST}

The relative corrosion resistance of the three wrought irons in the form of pipe was studied by laboratory tests carried out in tap water and in a $31 / 2$ per cent (by weight) sea-salt solution. The results of such tests, of course, are not necessarily indicative of the behavior of the materials in service. However, under service conditions which approximate in most respects the essential conditions of the tests, the service behavior of materials would be expected to follow the indications of the test results. Short pieces of the three kinds of pipe, varying from $1 \frac{1 / 4}{4}$ to $1 \frac{3 / 4}{4}$ inches in length, were used as specimens. The sawed ends of samples were ground smooth on an emery wheel and the samples were then cleaned free from grease by hot soapsuds, rinsed in hot water, dried and weighed. No attempt was made to remove adhering scale; for example, by pickling, as it was desired to test the materials in a condition approaching as nearly as possible the commercial condition.

The tests carried out in both solutions consisted of the following: (a) Immersion in the still liquid without any (intentional) aeration, (b) immersion in the liquid through which air was passed, and (c) repeated immersion or "wet-and-dry" test. The ends of most of the specimens, after weighing, were covered with a coating of aluminumpigmented "rubber cement" (thermoprene). It was noticed, however, after 24 hours' exposure that there was evidence of some corrosion on the ends and, since no marked difference was noted in the behavior of these samples and of those in which the ends were left bare, it may be concluded that the corrosion losses observed represent the attack on the cut ends as well as on the surfaces of the pipe. Twelve specimens from each of the three kinds of pipe (four specimens from three different pipe lengths) were tested in the two liquids with each of the three testing methods given above.

In carrying out the simple immersion tests, without aeration, eight samples, in two sets of four placed end to end and held in place by means of glass rods extending through the pipe, were immersed in the liquid to a depth of approximately 3 inches. Large glass battery jars were used as containers (fig. 11), and water was added from time to time to maintain the initial volume ( 4 liters) of the liquid. In carrying out the immersion tests, with aeration, the samples were placed end to end within a rubber tubing (inside tube of a bicycle tire), the whole being immersed in the liquid in a large container. Air was bubbled slowly through the inside of the assembled specimens during the daytime (eight hours). The repeated immersion or "wet-anddry" tests were carried out by means of the apparatus shown in Figure 12 ; a rather complete description of which has already been 


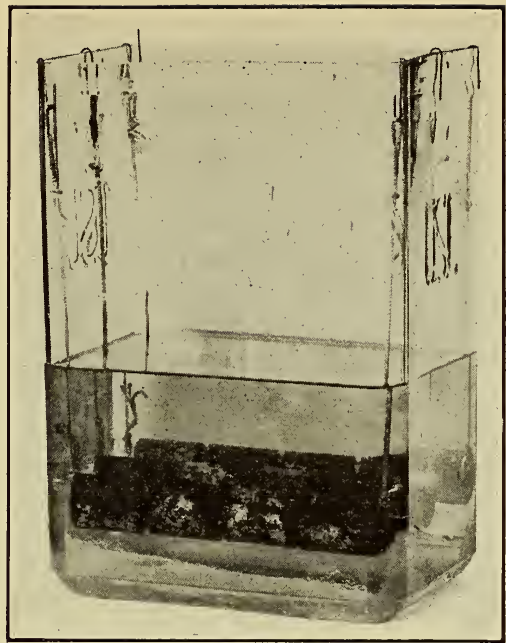

FIguRE 11.-Simple immersion method of carrying out the corrosion tests in unaerated solutions

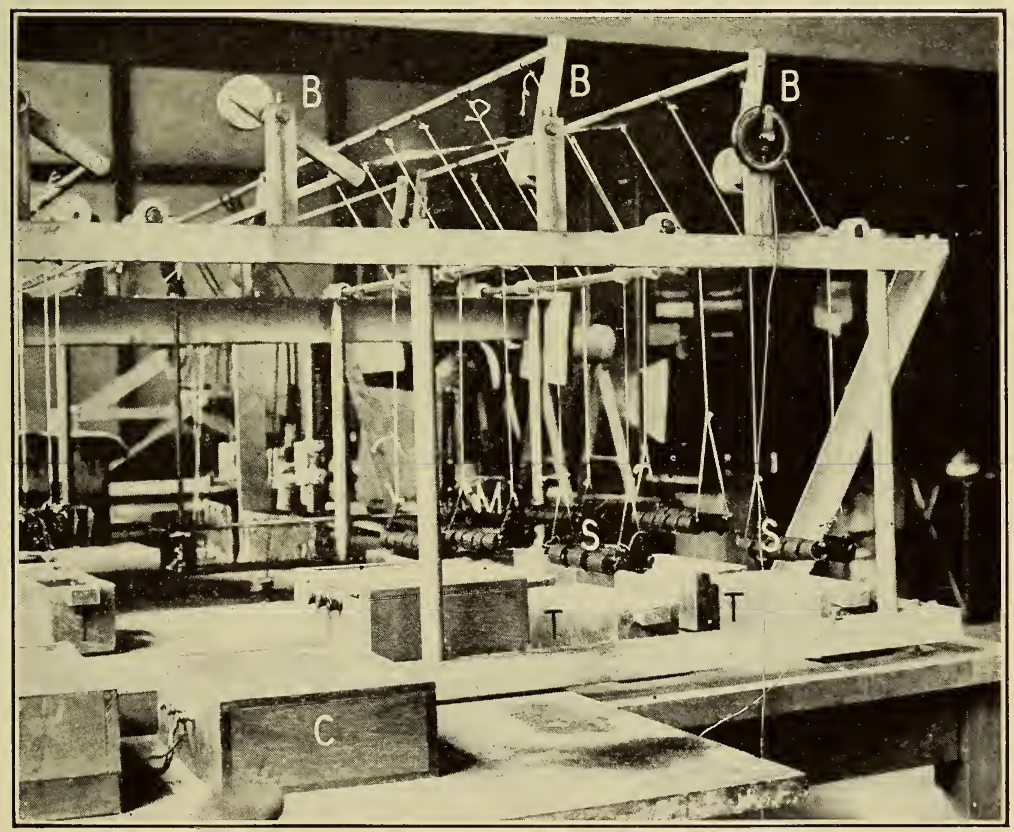

FIGURE 12.-Apparatus used for the wet-and-dry corrosion tests

By means of the clock, $C$, the motor, $M$, is started at 15 -minute intervals. The bell cranks, $B$, are turned and the specimens, $S$, are dipped into the solution in the tanks, $T$, and then raised into the air. 


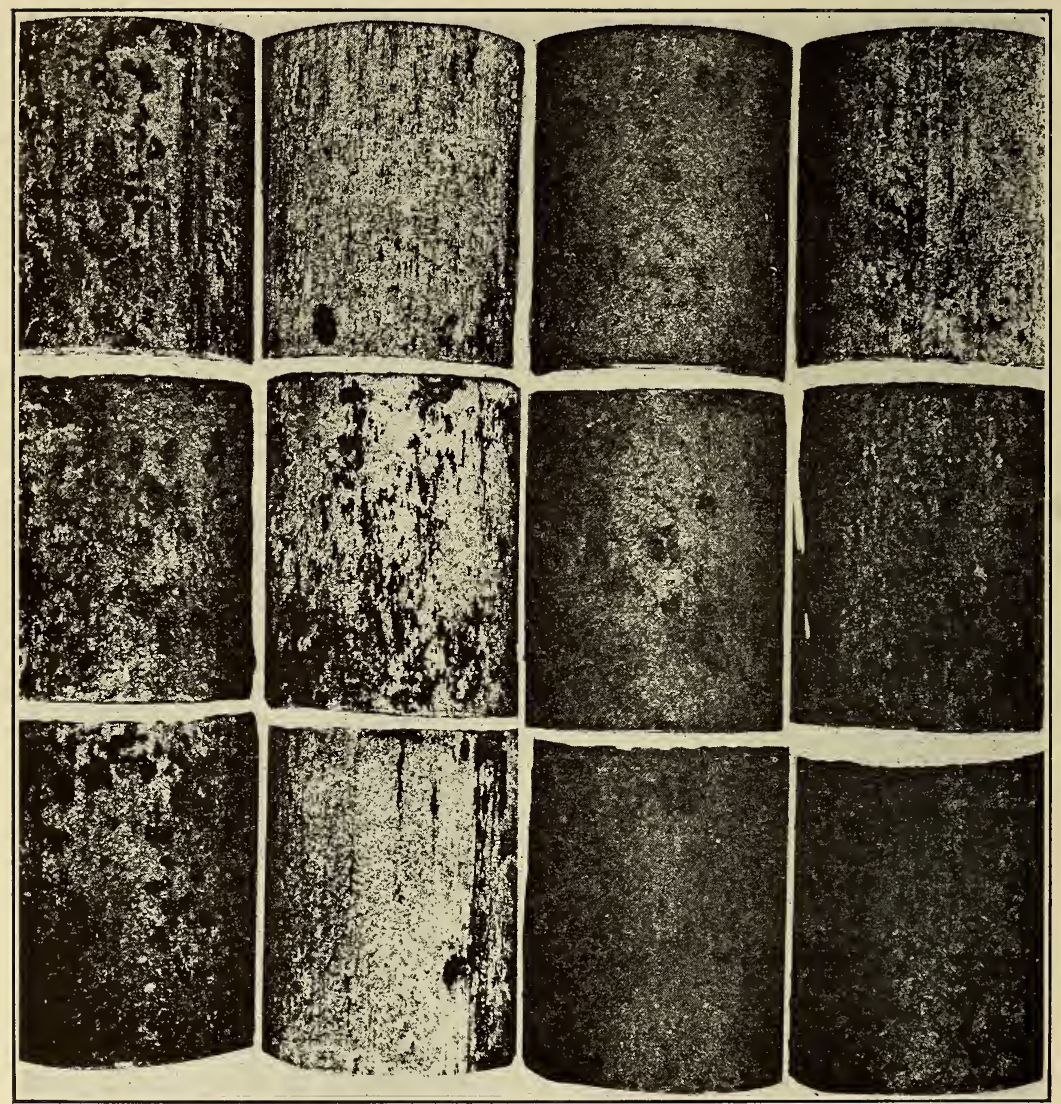

FIGURE 13.-Appearance of the outer surface of wrought-iron pipe after corrosion

The pipe in the upper horizontal row is hand-puddled wrought iron; that in the middle row, newprocess iron; and that in the third, the composite iron made from equal amounts of the other two. The specimens in the first vertical row at the left were corroded by simple immersion in tap water; those in the next, by immersion in $31 / 2$ per cent sea-salt solution; those in the third row, by the wet-and-dry method in tap water; and those in the right-hand row, by the wet-and-dry method in sea-salt solution. 
B. S. Journal of Research, RP124]

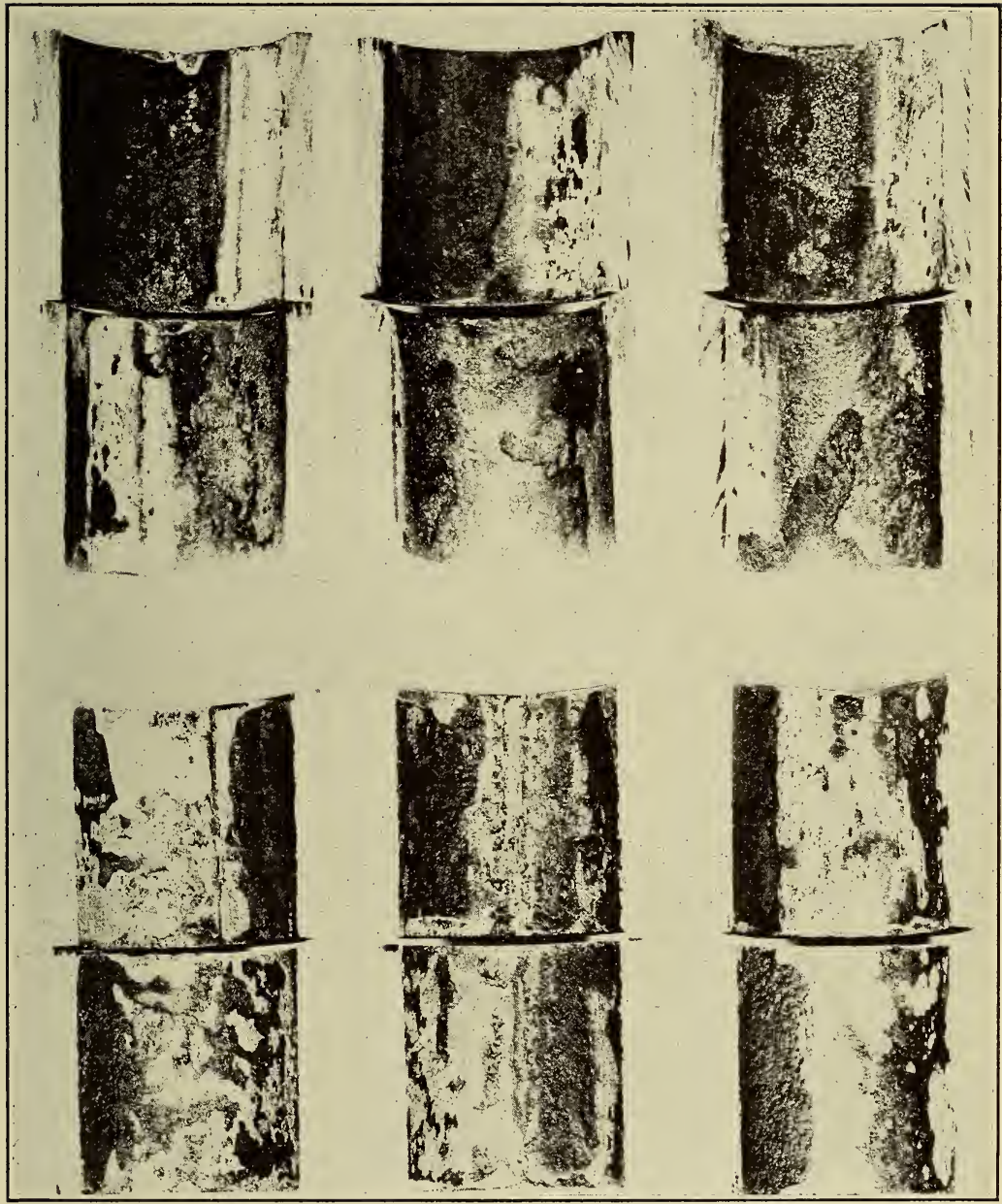

'FIGURE 14.-Appearance of the inner surface of wrought-iron pipe after corrosion by immersion in aerated solutions

Each group of two represents the two halves of the specimen split lengthwise. The specimens are arranged in vertical rows according to the kind of iron as follows (beginning at the left): Newprocess iron, composite iron, hand-puddled iron. The specimens in the upper double row were corroded in sea-salt solution, those in the lower double row in tap water. 
B. S. Journal of Research, RPI24

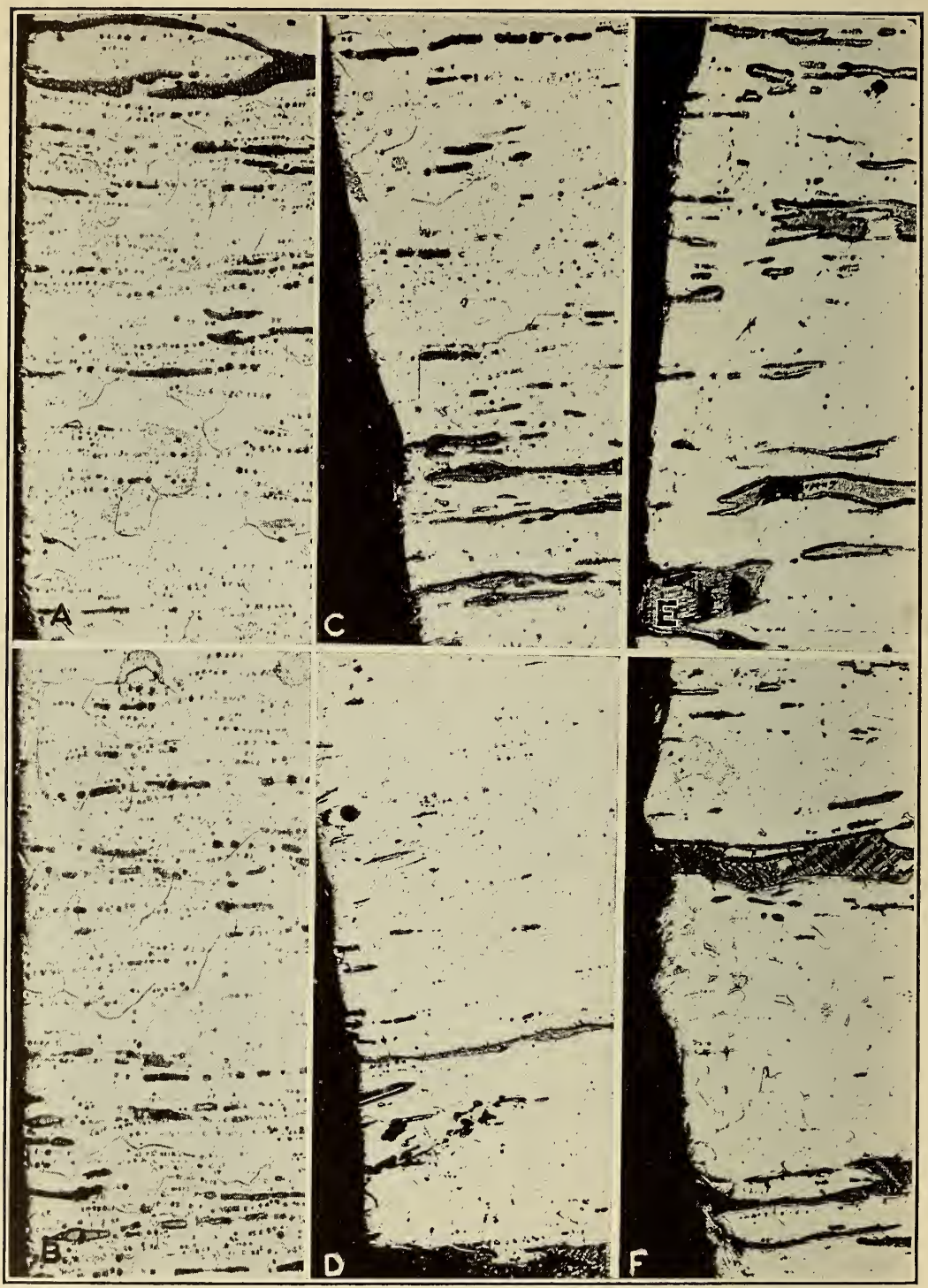

FIGURE 16.-Relation of the corrosive attack on "cut" ends of wrought-iron pipe, after simple immersion, to the microstructure, $\times 100$, reduced to $\times 75$

The micrographs represent longitudinal sections of the pipe at the corroded end, which is shown at the left in each case. $a, b$, New-process iron in tap water and $31 / 2$ per cent sea salt solution, respectively; $c, d$, hand-puddled iron in tap water and $31 / 2$ per cent sea-salt solution, respectively; $e, f$, the composite iron in tap water and $31 / 2$ per cent sea-salt solution, respectively. 
B. S. Journal of Research, RP124

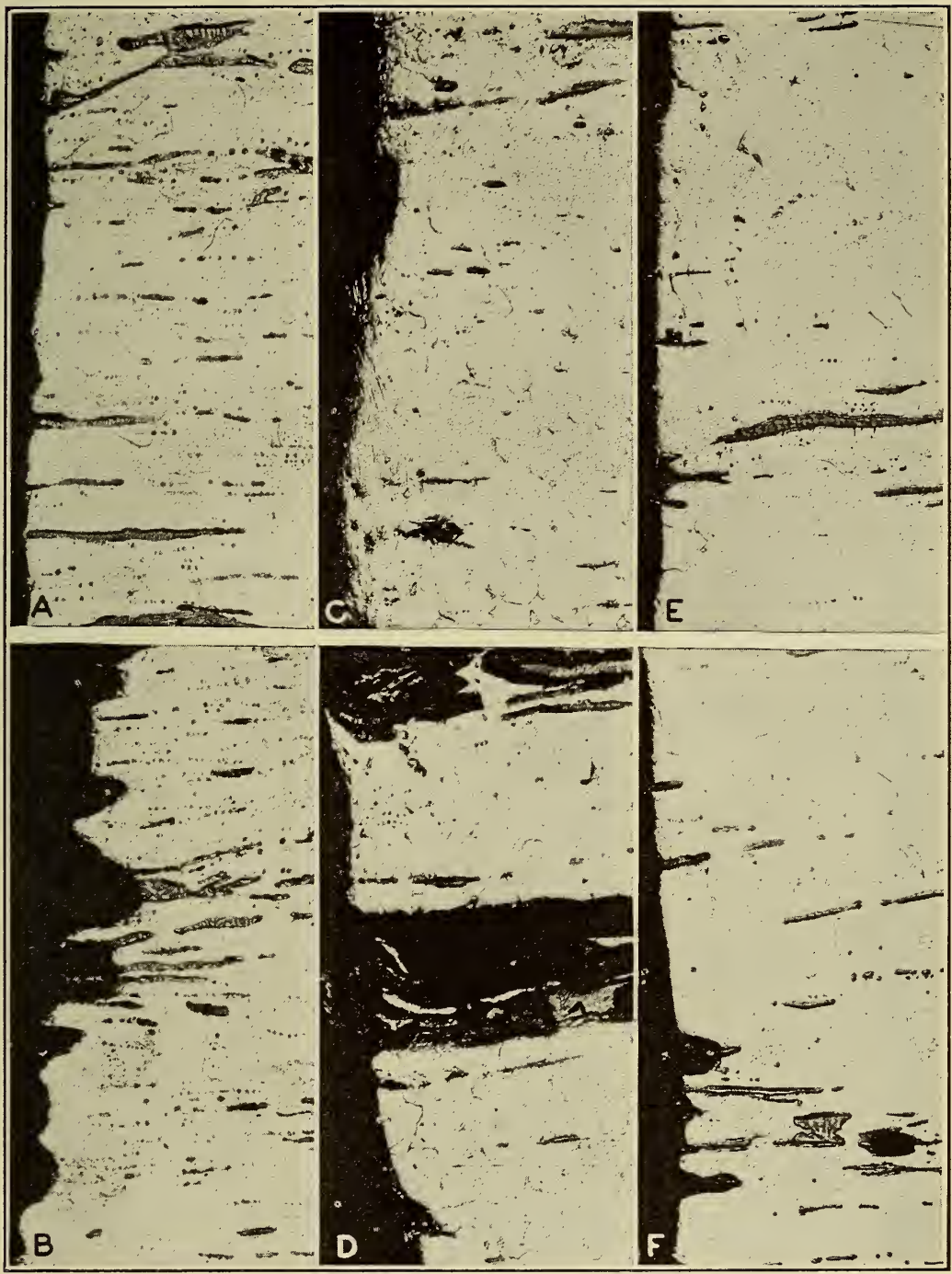

FigUre 17.-Relation of the corrosive attack on "cut" ends of wrought-iron pipe after immersion in aerated solutions, to the microstructure, $\times 100$, reduced to $\times 75$

The micrographs represent longitudinal sections of the pipe at the corroded end, which is shown at the left in each case. $a, b$, New-process iron in tap water and $3 \frac{1}{2}$ per cent sea-salt solution, respectively; $c, d$, hand-puddled iron in tap water and $31 / 2$ per cent sea-salt solution, respectively; $\ell e, f$, the composite iron in tap water and $3 \frac{1}{2}$ per cent sea-salt solution, respectively. 

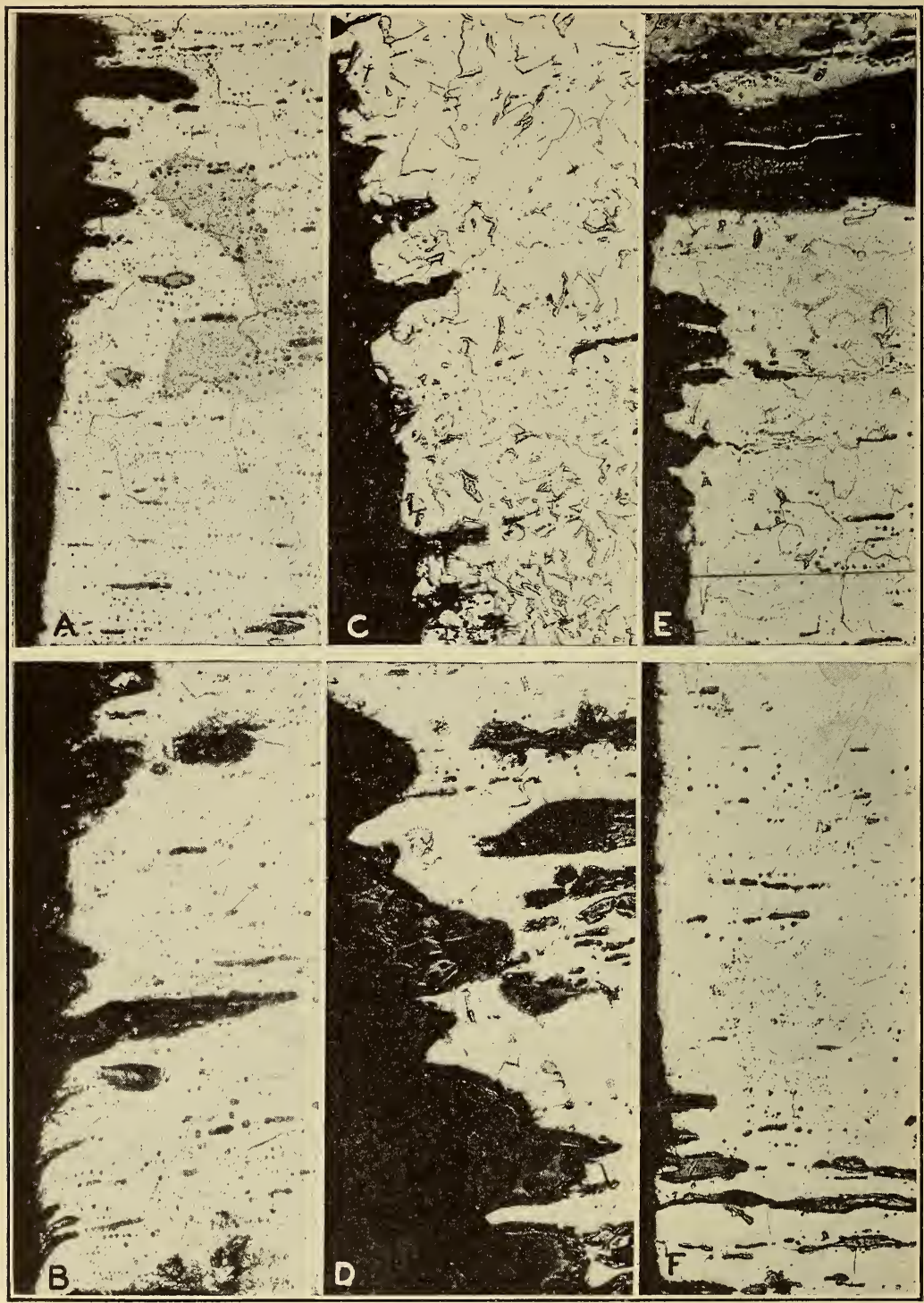

Figure 18.-Relation of the corrosive attack on "cut" ends of wrought-iron pipe after corrosion by the wet-and-dry method, to the microstructure, $\times 100$, reduced to $\times 75$

The micrographs represent longitudinal sections of the pipe at the corroded end, which is shown at the left in each case. $a, b$, New-process 1ron in tap water and $3 \frac{1}{2}$ per cent sea-salt solution, respectively; $c, d$, hand-puddled tron in tap water and $3 \frac{1}{2}$ per cent sea-salt solution, respectively; $e, f$, the composite iron in tap water and $3 \frac{1}{2}$ per cent sea-salt solution, respectively. 
published. ${ }^{8}$ The specimens, which were supported by cords and glass rods as shown, were wet at 15-minute intervals with the liquid (tap water or sea-salt solution) by being dipped momentarily into the solution and then raised into the air. The specimens were immersed in the liquid approximately one minute at each immersion. The corrosion tests were allowed to continue for three months (92 days) except in the case of a few specimens which were examined at the end of 2 months. No attempt was made to remove the corrosion product during the period of corrosion. The tests were carried out during the three summer months at room temperature. At the end of the 3 -month period the specimens were cleaned free from the corrosion product, washed first in hot water, and then in alcohol, dried, and weighed. The loss of weight per unit of surface area was used as a basis of comparison.

No marked or important difference in the character of the corrosion product on the different kinds of iron was noted. The character of the corrosion product differed decidedly, however, according to the conditions under which the corrosive attack had occurred. A loose flocculent deposit which could be removed very readily was formed on the specimens which were corroded by simple immersion (unaerated) in both tap water and salt solution. The deposit which formed on the specimens corroded in the aerated solutions was much more adherent, but was readily removed by means of a bristle brush. In the case of the wet-and-dry tests, however, the character of the scale formed was decidedly different from that in the other tests and was removed only with difficulty. On the specimens corroded in sea-salt solution the scale, though much more adherent than in the other cases, was coarse and was removed by vigorous brushing. The scale on the specimens corroded by repeated immersion in tap water, however, though thin, was smooth and very adherent. It was removed with a bristle brush only with great difficulty even after the specimens had been allowed to remain in a 10 per cent (by weight) solution of ammonium citrate for two days. The appearance of the outer and inner corroded surfaces of representative specimens of the various tests is shown in Figures 13 and 14, respectively.

A summary of the average corrosion loss of the three kinds of pipe when corroded in different ways in tap water and in salt solution is given in Table 16 and a graphical summary of the results of the corrosion tests in Figure 15. As shown by the data in Table 16, there appears to be a very slight difference in the corrosion rate of the hand-puddled and the new-process wrought iron in favor of the hand-puddled product. It is also very apparent that the method by which the attack is brought about is a much more important

${ }^{8}$ H. S. Rawdon and E. C. Groesbeck, Effect of the Testing Method on the Determination of Corrosion Resistance, B. S. Tech. Paper No. 367; 1928. 
factor in determining the corrosion resistance than is any difference in the character of the iron resulting from the process by which it has been prepared. The appearance of the corroded specimens shown in Figures 13 and 14 is typical of results obtained with the different materials corroded under different conditions. Corrosion on the cut ends of wrought iron in the form of pipe or bar is

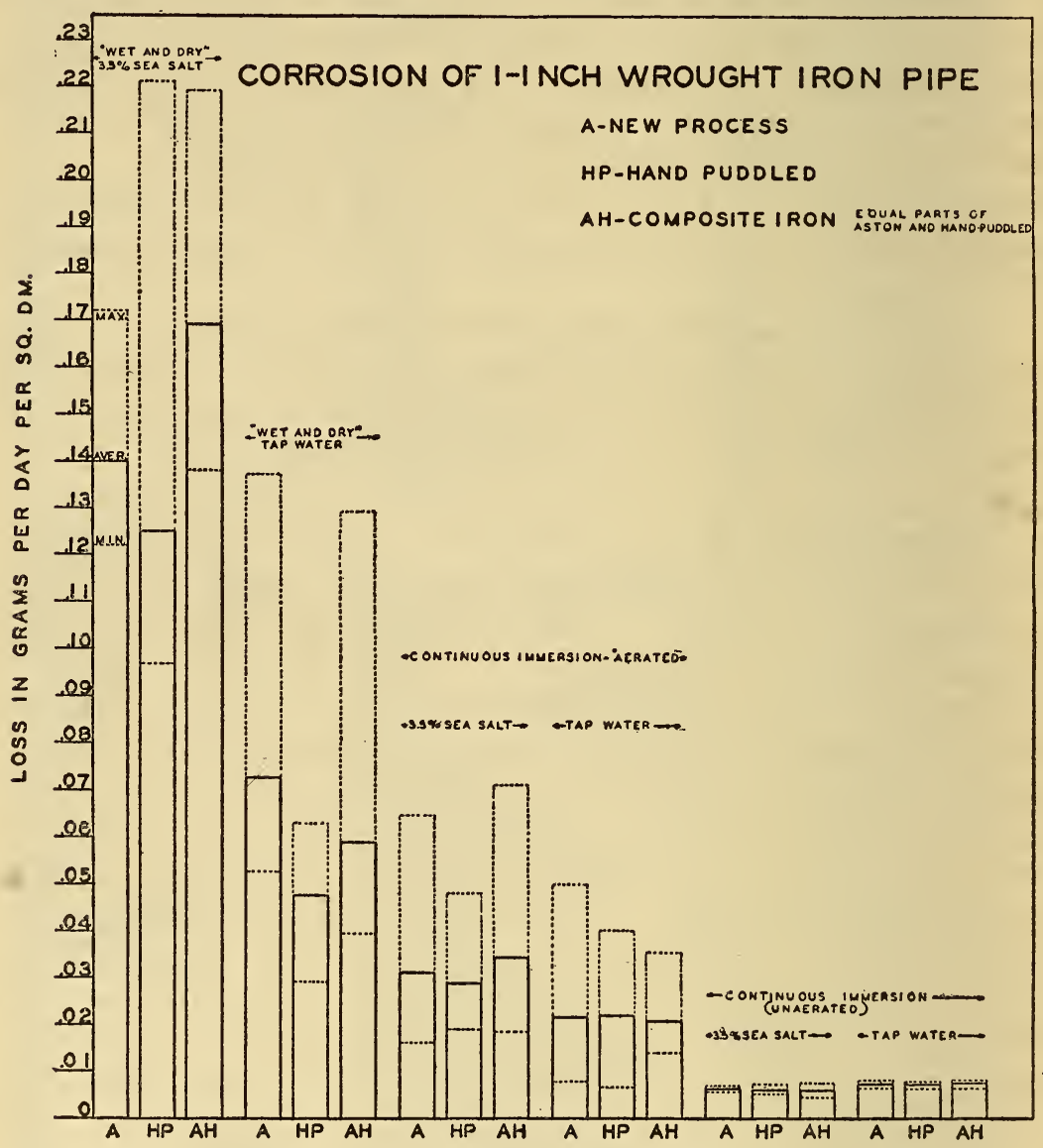

FIgURE 15.-Summary of the results of the corrosion tests

The maximum and minimum values are shown by the dotted lines.

generally conceded to be more severe than on the rolled surface of the same material. The character and extent of the corrosive attack on the cut ends of some of the specimens used in the present tests are shown in Figures 16, 17, and 18. These micrographs are further confirmation of the conclusion based upon the other corrosion data that the conditions under which the corrosive attack occurred played a greater part in determining the corrosion loss than did differences in the material itself. 
TABLE 16.-Average ${ }^{1}$ corrosion losses of wrought-iron pipe in accelerated laboratory tests of three months' duration

[ $\mathrm{A}=$ iron made by the Aston process; $\mathrm{HP}=$ hand-puddled iron; $\mathrm{AHP}=$ composite iron containing equal amounts of $\mathrm{A}$ and $\mathrm{HP}]$

\begin{tabular}{|c|c|c|c|c|c|c|}
\hline \multirow{3}{*}{ Material } & \multicolumn{6}{|c|}{ Corrosion method } \\
\hline & \multicolumn{2}{|c|}{ Simple immersion } & \multicolumn{2}{|c|}{ Immersion aerated } & \multicolumn{2}{|c|}{ "Wet-and-dry" } \\
\hline & $\begin{array}{c}\text { Sea-salt } \\
\text { solution? }\end{array}$ & $\begin{array}{c}\text { Tap } \\
\text { water }\end{array}$ & $\left|\begin{array}{c}\text { Sea-salt } \\
\text { solution } 2\end{array}\right|$ & $\begin{array}{c}\text { Tap } \\
\text { water }\end{array}$ & $\left|\begin{array}{c}\text { Sea-salt } \\
\text { solution }\end{array}\right|$ & $\begin{array}{c}\text { Tap } \\
\text { water }\end{array}$ \\
\hline $\begin{array}{l}\mathrm{H} \mathrm{P} \\
\mathrm{A} \mathrm{P}_{2}\end{array}$ & $\begin{array}{r}30.0063 \\
.0061 \\
.0059\end{array}$ & $\begin{array}{r}0.0074 \\
.0072 \\
.0077\end{array}$ & $\begin{array}{r}0.031 \\
.029 \\
.034\end{array}$ & $\begin{array}{r}0.0215 \\
.0217 \\
.0207\end{array}$ & $\begin{array}{r}0.140 \\
.125 \\
.168\end{array}$ & $\begin{array}{r}0.073 \\
.047 \\
.057\end{array}$ \\
\hline
\end{tabular}

1 See fig. 15 for the range of the determinations.

$231 / 2$ per cent solution, by weight.

${ }^{3} \mathrm{~g} / \mathrm{dm}^{2} /$ day.

\section{(b) ELECTROLYTIC SOLUTION POTENTIAL}

Coincident with the laboratory corrosion tests and before the results of these tests were known, a few simple tests were carried out to show the relative behavior of the two wrought irons when in electrical contact with each other and immersed in a solution. Small pieces of the skelp strip, 3 by $3 / 4$ inch, were used as specimens. These were cleaned by pickling, after which they were heated in an oven to approximately $200^{\circ} \mathrm{C}$. in order to drive off any hydrogen present. A copper wire was firmly attached to one end of the specimen and the junction of iron and copper, as well as the wire itself, coated with paraffin. A dilute solution of sodium sulphate (1 per cent, by weight) was used as the electrolyte in which the two iron specimens were immersed, "face to face," slightly more than 1 inch apart. A small potentiometer of the portable type was used to show the magnitude and direction of the e. m. f. which resulted when electrical contact was established between the two specimens.

There was considerable variation in the e. m. f. observed for several different couples as well as for the same couple used on different days, which is not surprising in view of the simple method used. The results were consistent, however, in one respect. The hand-puddled iron was, in all cases, the cathode of the couple; that is, for the conditions used, the "solution pressure" of the new-process iron was slightly higher than that of the comparison material.

\section{STRUCTURAL EXAMINATION}

Inasmuch as the structural condition of wrought iron is one of its most characteristic features, the comparison of the structure of the two types of iron was carried out in considerable detail. 


\section{(a) PIPE MATERIALS}

(1) BALL.-In Figures 19 and 20 is shown the appearance of portions of a wrought-iron ball as taken from the hand-puddling furnace and of a ball of the wrought iron made by the new process as it comes from the shotting cup. Only a very small portion of the entire ball is shown in either case. The microstructure of the two irons was examined on a considerable number of fragments in order to obtain a good idea of the average structure. Figures 21 and 22 are representative of the conditions found. Figure $21(a)$ and $(b)$ represents the average condition of the hand-puddled iron with respect to slag distribution. After etching, evidence of the presence of carbon was frequently found. The metal was quite "patchy" in this respect. The condition shown in micrograph $(b)$ is not unusual. Patches with a carbon content as high as that shown in (c) (fig. 21) appeared to be not uncommon and occasionally patches of iron in which the carbon was considerably above 1 per cent were found (fig. $21(d)$ ). The structural condition of the new-process iron in the ball was much more uniform than that of the hand-puddled iron with which it was compared. The degree to which the continuity of the iron matrix was broken up during the shotting operation and the slag was mechanically incorporated with the iron is surprising. In addition to the coating of slag covering the globules of iron formed by the shotting operation, the interior of globules showed an intimate mixture of the slag often suggestive of a dendritic arrangement (fig. 21 (b)). No evidence of the presence of carbon was found upon etching and the structure in this respect afforded a striking contrast to the other type of iron.

(2) Muck BAR.-In Figure 23 is shown the microscopic appearance of the two irons after being rolled into muck bar. The less uniform condition of the hand-puddled iron, as shown by the larger slag areas and the presence of the "carbon streaks," which were revealed by etching polished sections, is evident. The microstructure of the muck bar, as shown in Figures 24, 25, and 26, was found to be in agreement with that of the ball. The hand-puddled iron showed occasional slag streaks which were considerably larger than any found in the other iron and streaks of metal with a rather high carbon content were not uncommon. No carbon streaks were found in the muck bar of the new-process wrought iron and the average distribution of the slag threads was considered to be somewhat more uniform. In the comparison of the two irons, here and elsewhere, the examination of transverse sections was used more than longitudinal sections, since a transverse section gives a much better index of the distribution of the slag streaks than the other does. 

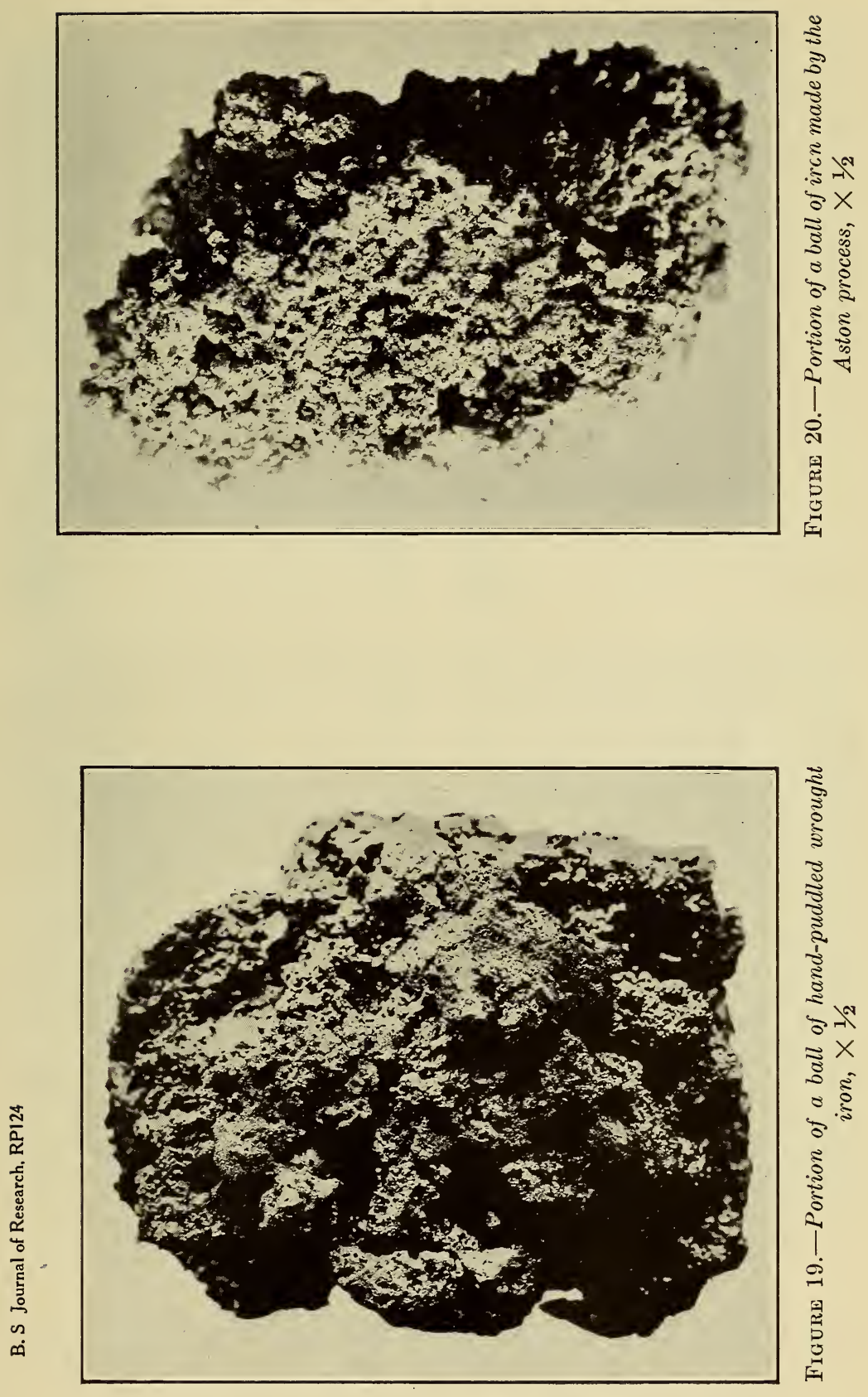
B. S. Journal of Research, RP124

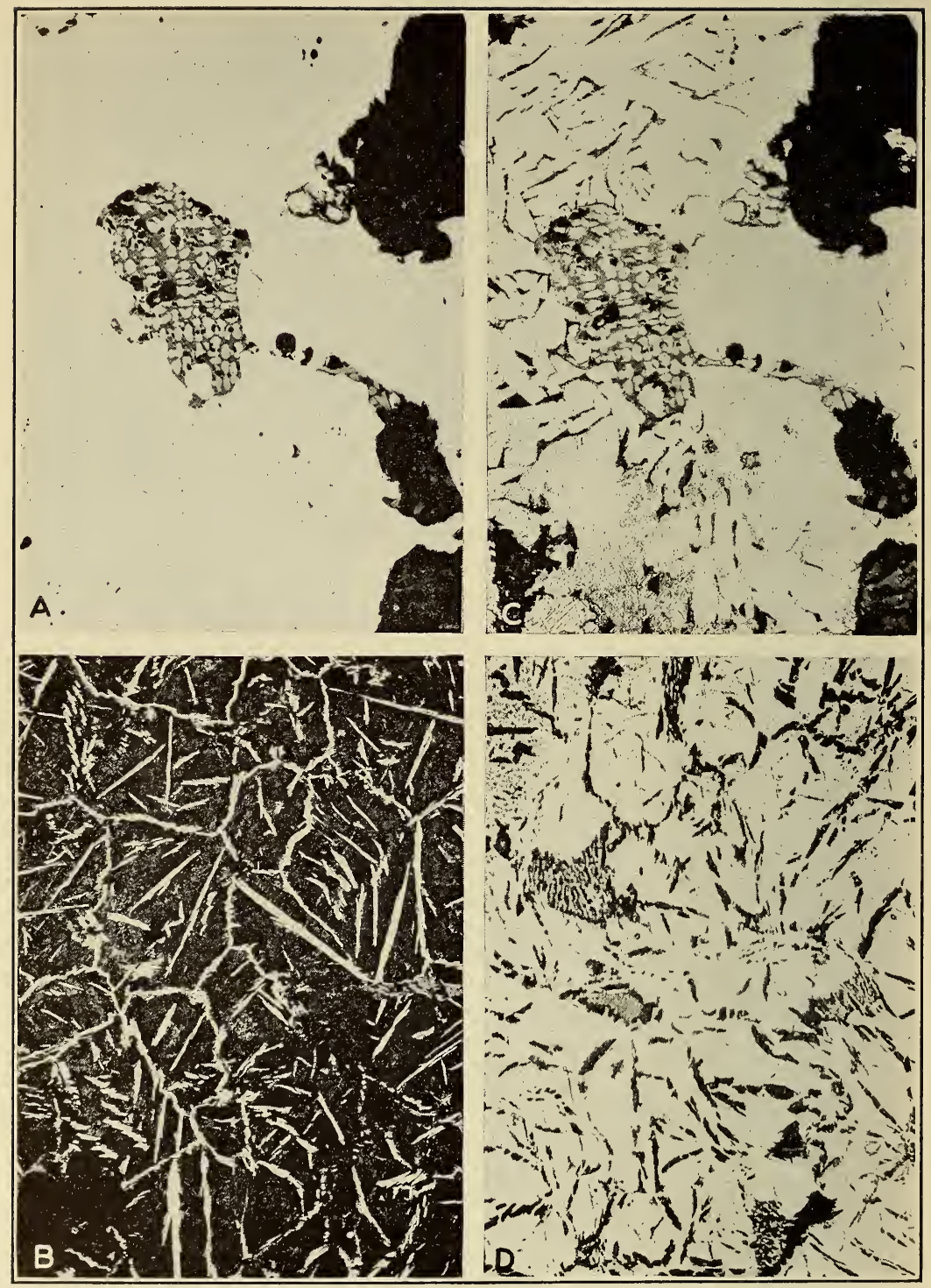

Figure 21.-Microstructure of hand-puddled iron in the ball stage, $\times 100$, reduced to $\times 75$

$a$, Unetched section, typical of the structure of much of the ball; $b$, same, etched 'with picric acid solution. Note the carbon in the form of small islands of pearlite; $c$, portion of the same ball having a carbon content over 1 per cent, etched with picric acid solution; $d$, another portion of the same ball showing a high carbon content, etched with hot alkaline sodium picrate solution. 


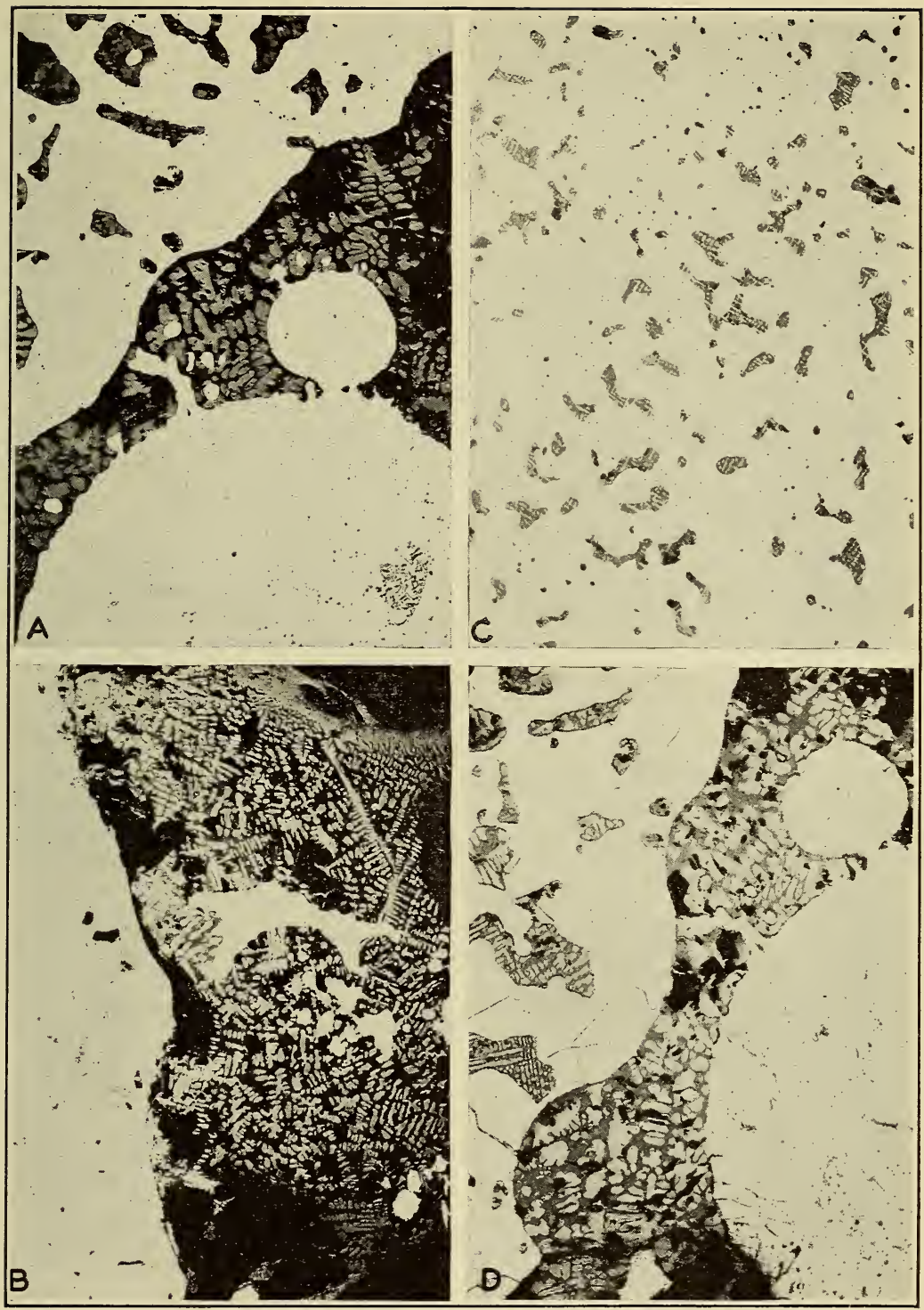

FIGURE 22.-Microstructure of wrought iron made by the new process, in the ball stage, $\times 100$, reduced to $\times 75$

$a, b$, and $c$ represent areas from two different balls, unetched. Micrograph $c$ shows the intimate mixture of slag and iron within what may appear to be a solid lump of metal, and $d$, same area as $a$, etched with picric acid solution. No carbon was revealed. 
B. S. Journal of Research, RP124

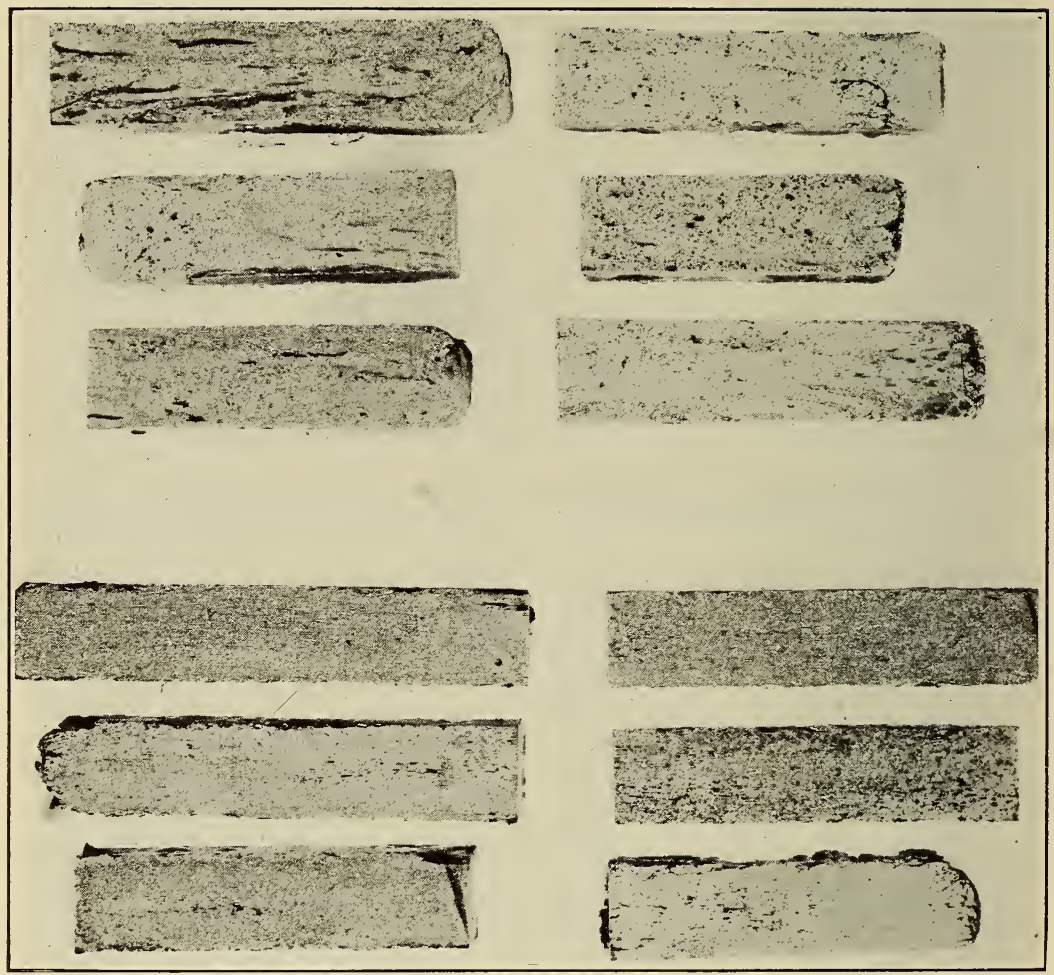

FIGURE 23.-Microstructure of wrought iron in the muck-bar state, $\times 2 / 3$

The upper group represents different samples of hand-puddled iron; the lower, corresponding samples of the new-process iron. Etchant, 4 per cent nitric acid solution. 
B. S. Journal of Research, RP124

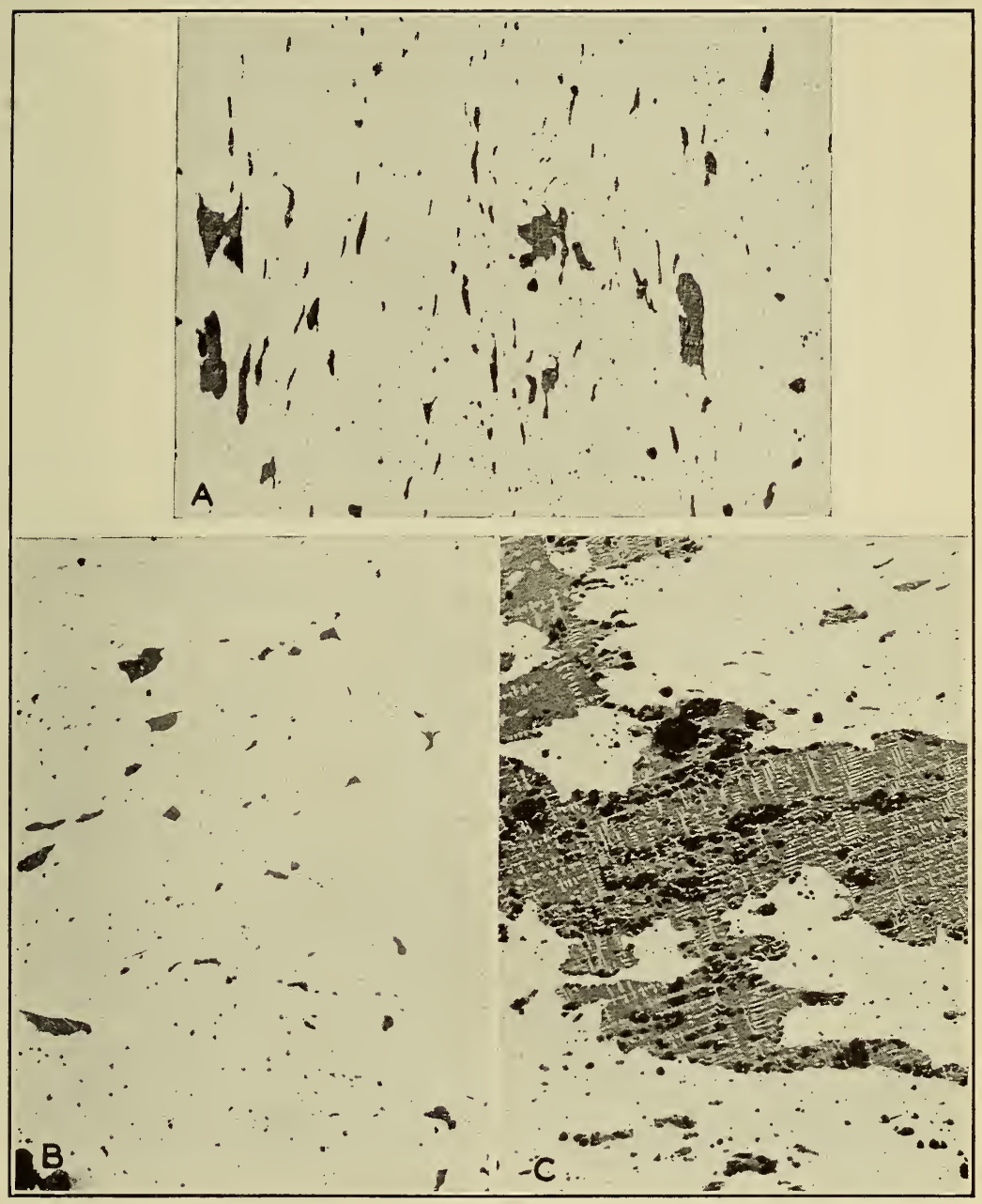

FIgURE 24.-Microstructure of hand-puddled wrought iron in the muck-bar state, $\times 100$, reduced to $\times 75$

The micrographs are of transverse sections, unetched. $a$, Area representing the average size of slag threads (transverse sections) and their distribution; $b$, shows an area with the slag threads as small as were found; and $c$, transverse section of a large slag thread. Such threads were not uncommon. 


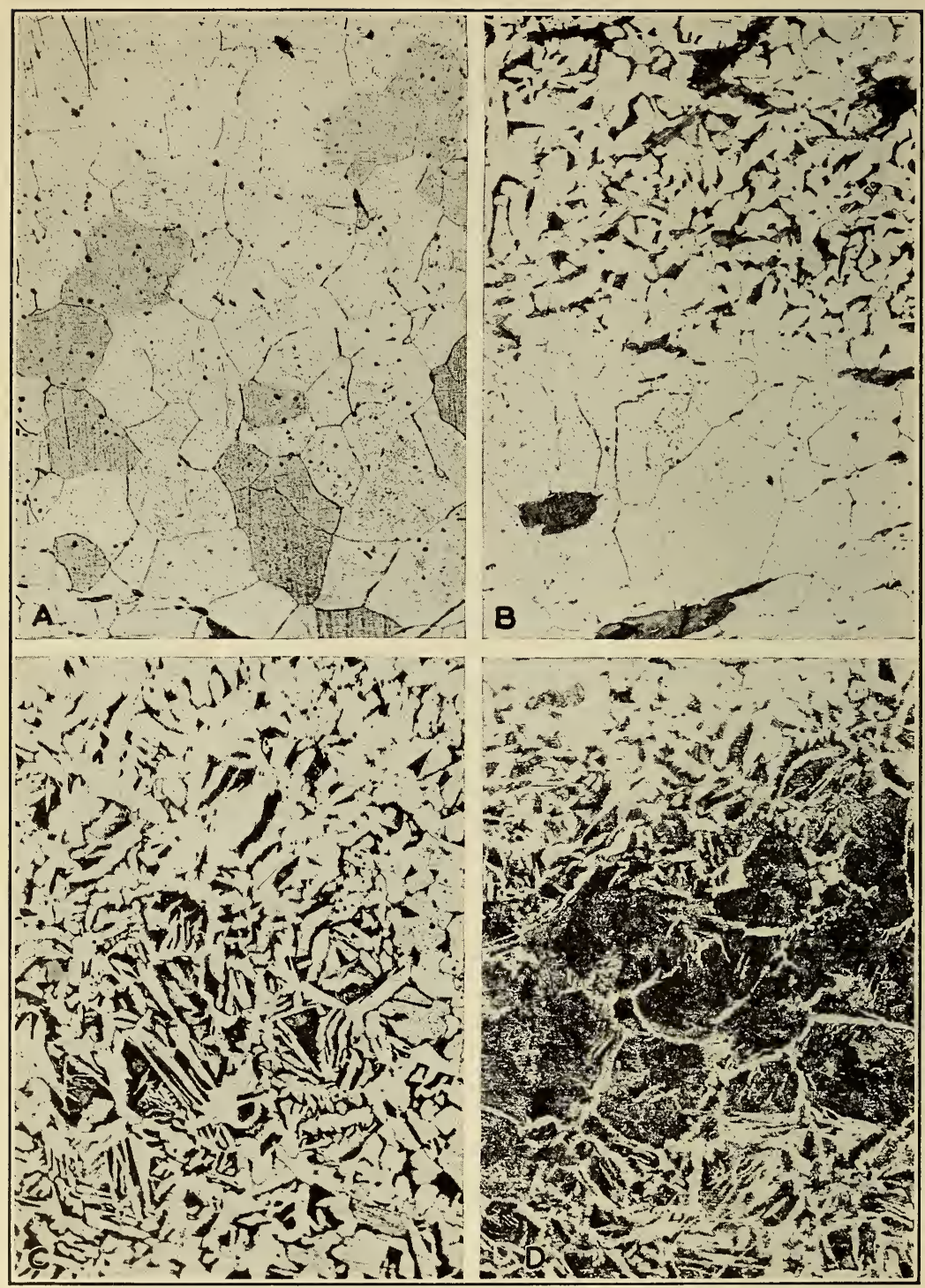

FIGURE 25.-Microstructure of hand-puddled wrought iron in the muck-bar stage, $\times 100$, reduced to $\times 75$

The specimens (transverse sections) were etched with an alcoholic solution of picric acid. $a, \mathrm{~A}$ large part of the material showed no carbon, as is represented here; and $b$, $c$, and $d$, "carbon streaks" of increasing carbon content found scattered throughout the muck bar. 


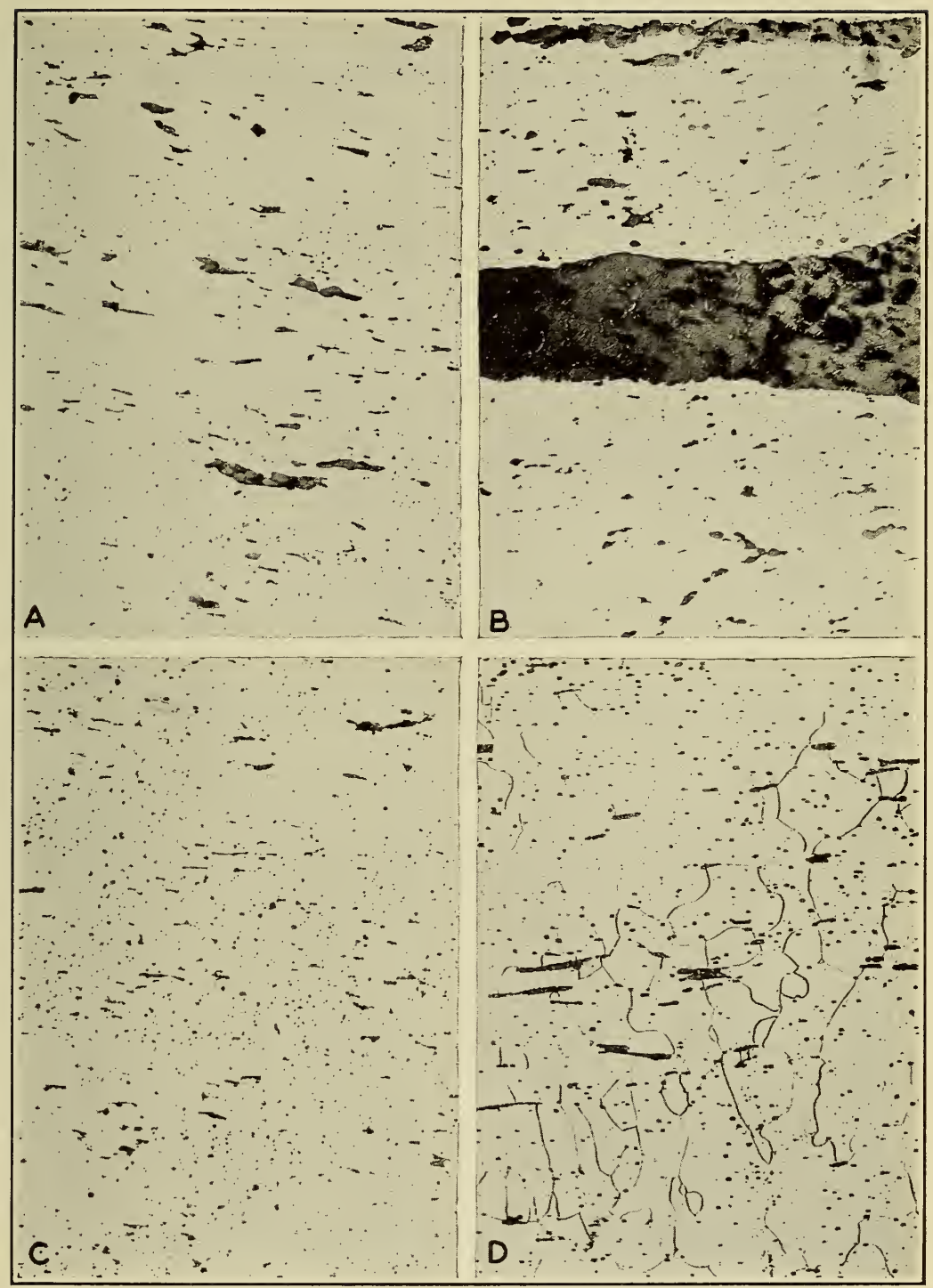

FIGURE 26.-Microstructure of wrought iron made by the new process, in the muck-bar state, $\times 100$, reduced to $\times 75$

The micrographs show transverse sections, unetched, except in $d$, which was etched with alcoholic picric acid solution. $a$, Area representing the average size and distribution of slag threads; $b$, area showing some of the largest slag threads found; $c$, area showing small slag threads; and $d$, appearance after etching. No carbon was found. 


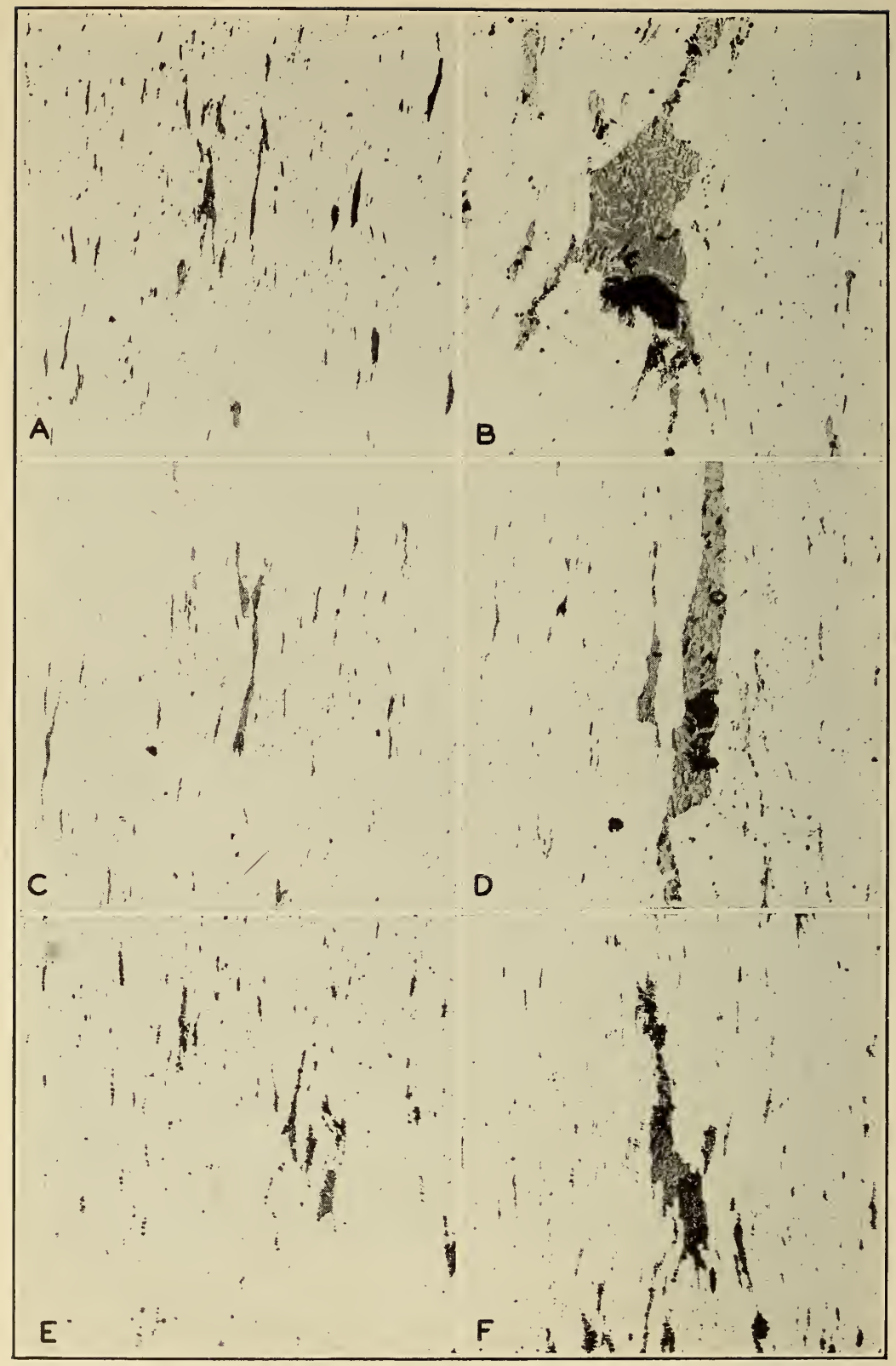

FIGURE 27.-Microstructure of wrought iron in the form of pipe skelp, $\times 100$, reduced to $\times 75$

The micrographs are of unetched transverse sections and represent the average condition of the slag (left) and some of the large slag threads (right). $a, b$, Hand-puddled iron; $c, d$, new process; and $e, f$, composite iron. 


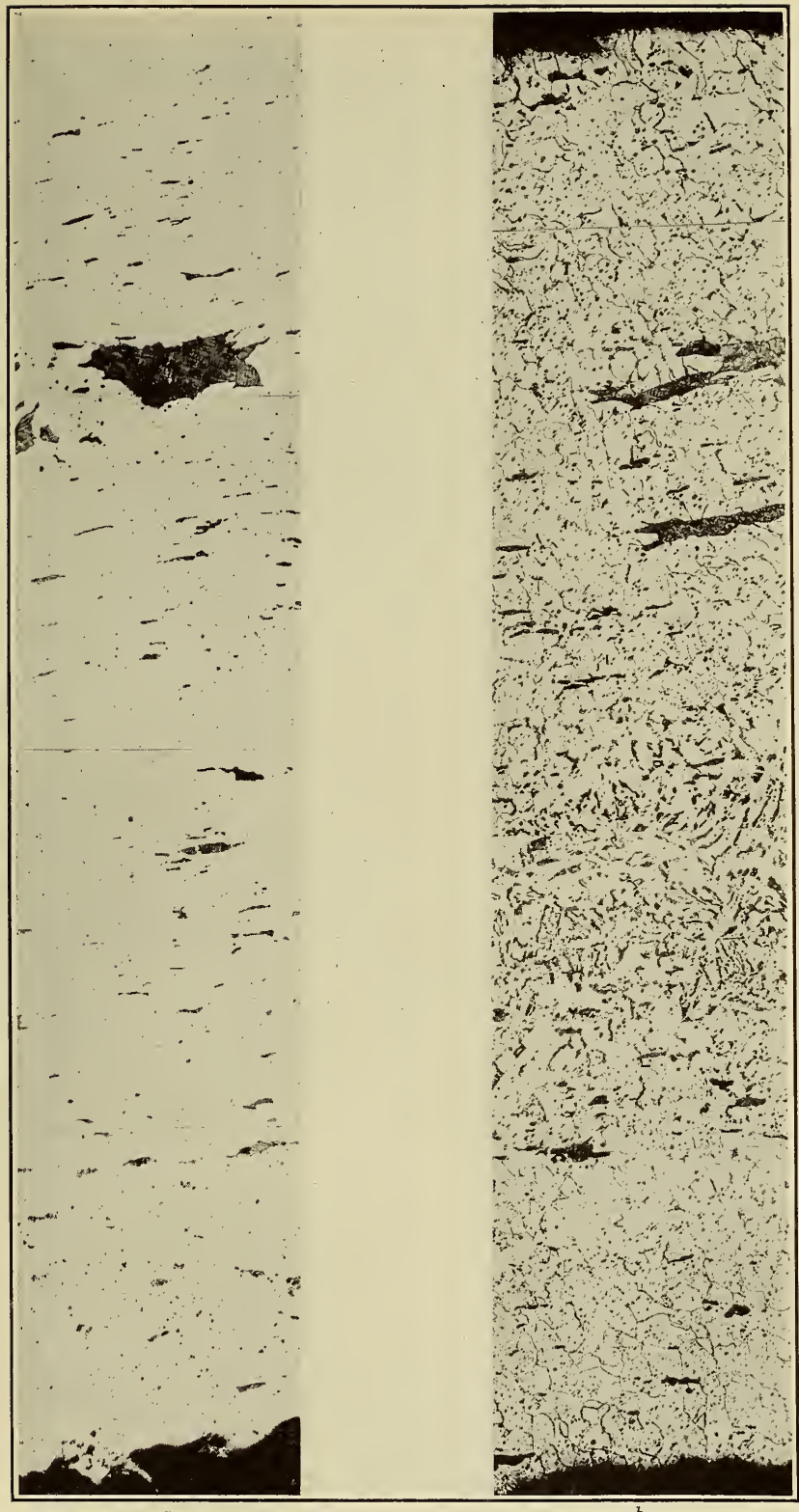

FIGURE 28.-Microstructure of hand-puddled wrought-iron pipe, $\times 100$, reduced to $\times 50$

Transverse sections of the entire wall of two 1-inch pipes are shown. $a$, Unetched; $b$, etched with alcoholic picric-acid solution. Note the pearlite (carbon) in $b$. 
B. S. Journal of Research, RP124

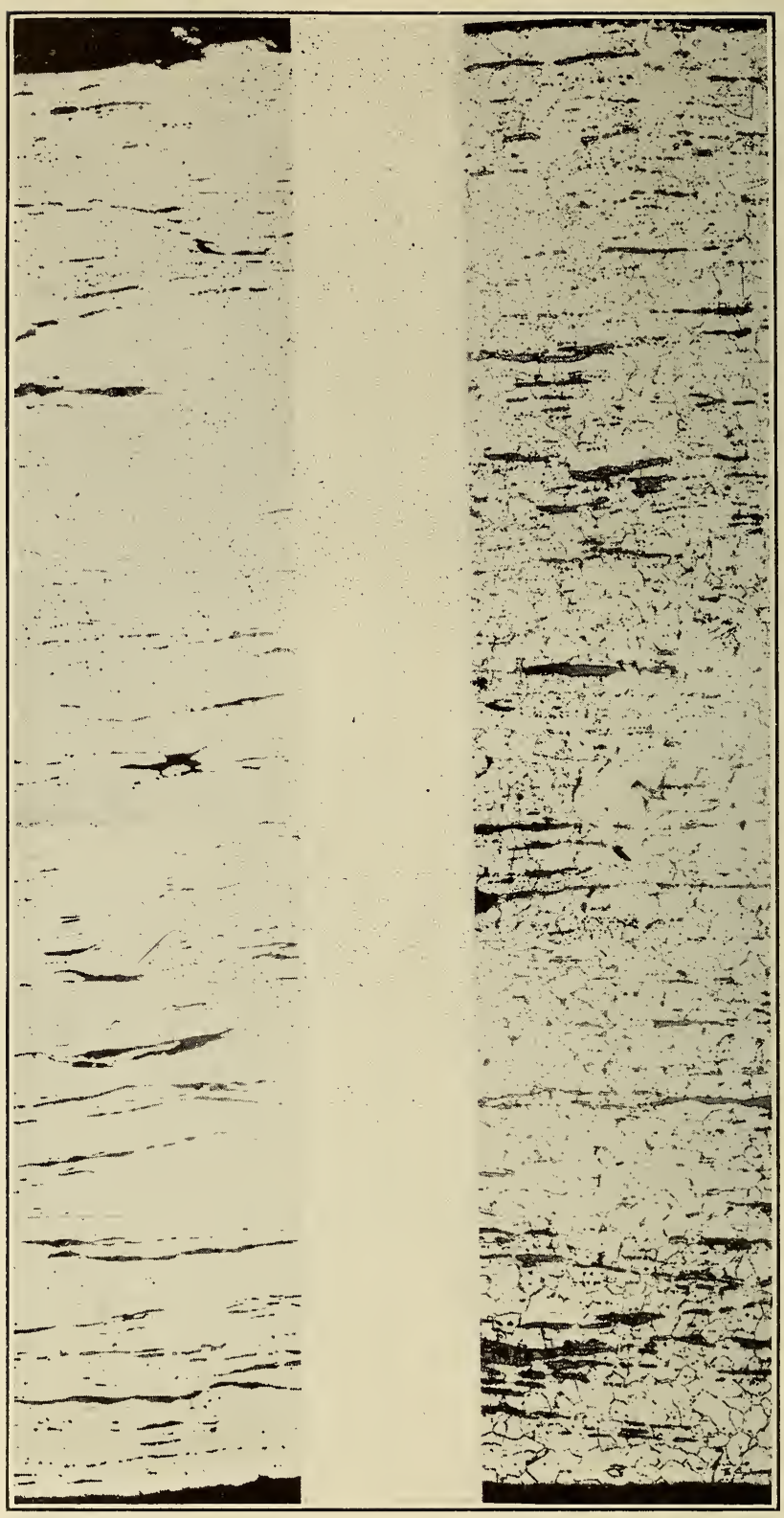

FIGURE 29.-Microstructure of hand-puddled wrought-iron pipe, $\times 50$

Longitudinal sections, unetched and etched, of the entire wall of a 1-inch pipe. 


\section{B. S. Journal of Research, RP124}

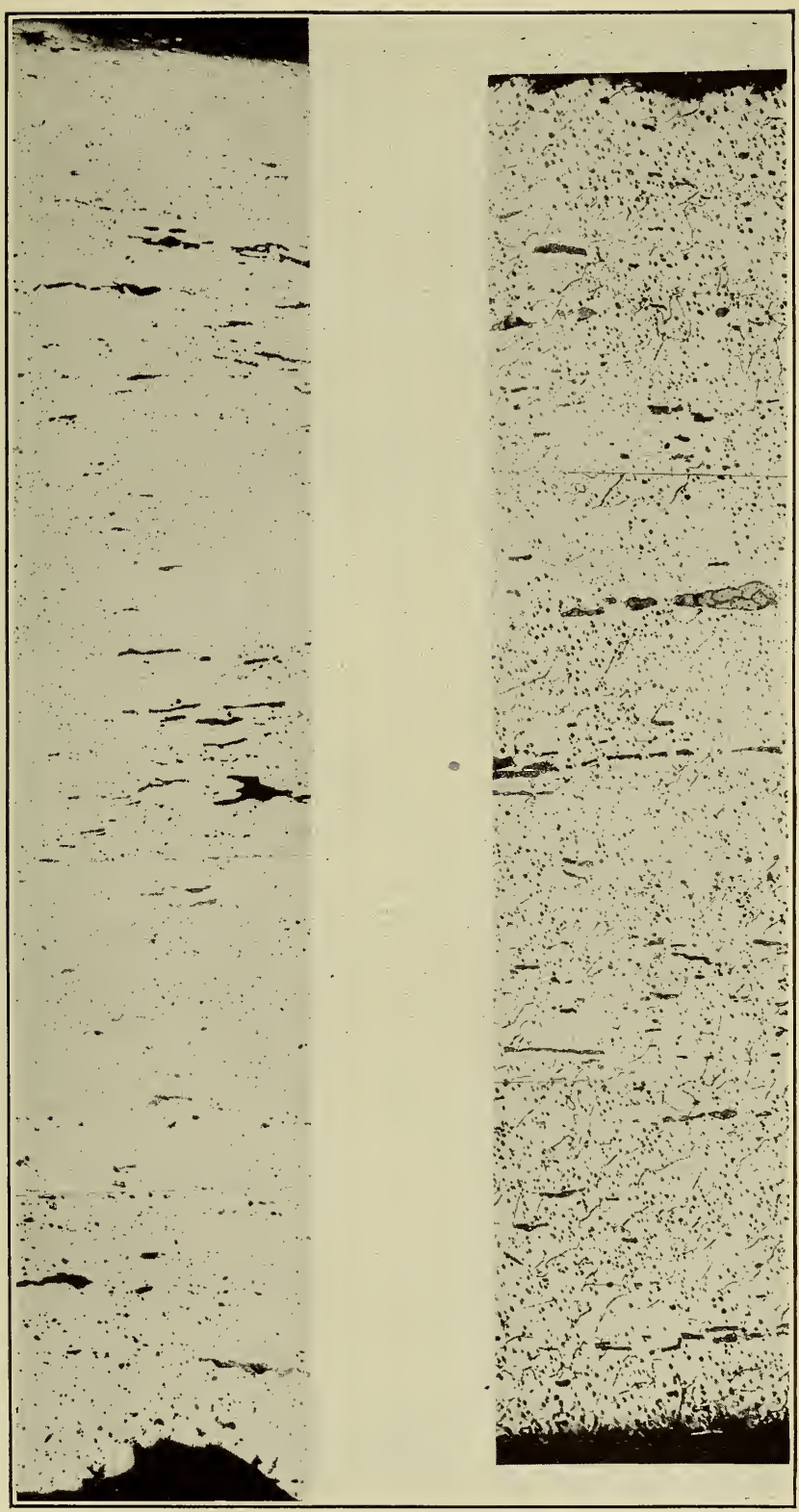

FIgURe 30.-Microstructure of wrought-iron pipe made by the Aston process, $\times 100$, reduced to $\times 50$

Transverse sections, unetched and etched, of the entire wall of two 1-inch pipes. 
B. S. Journal of Research, RP124

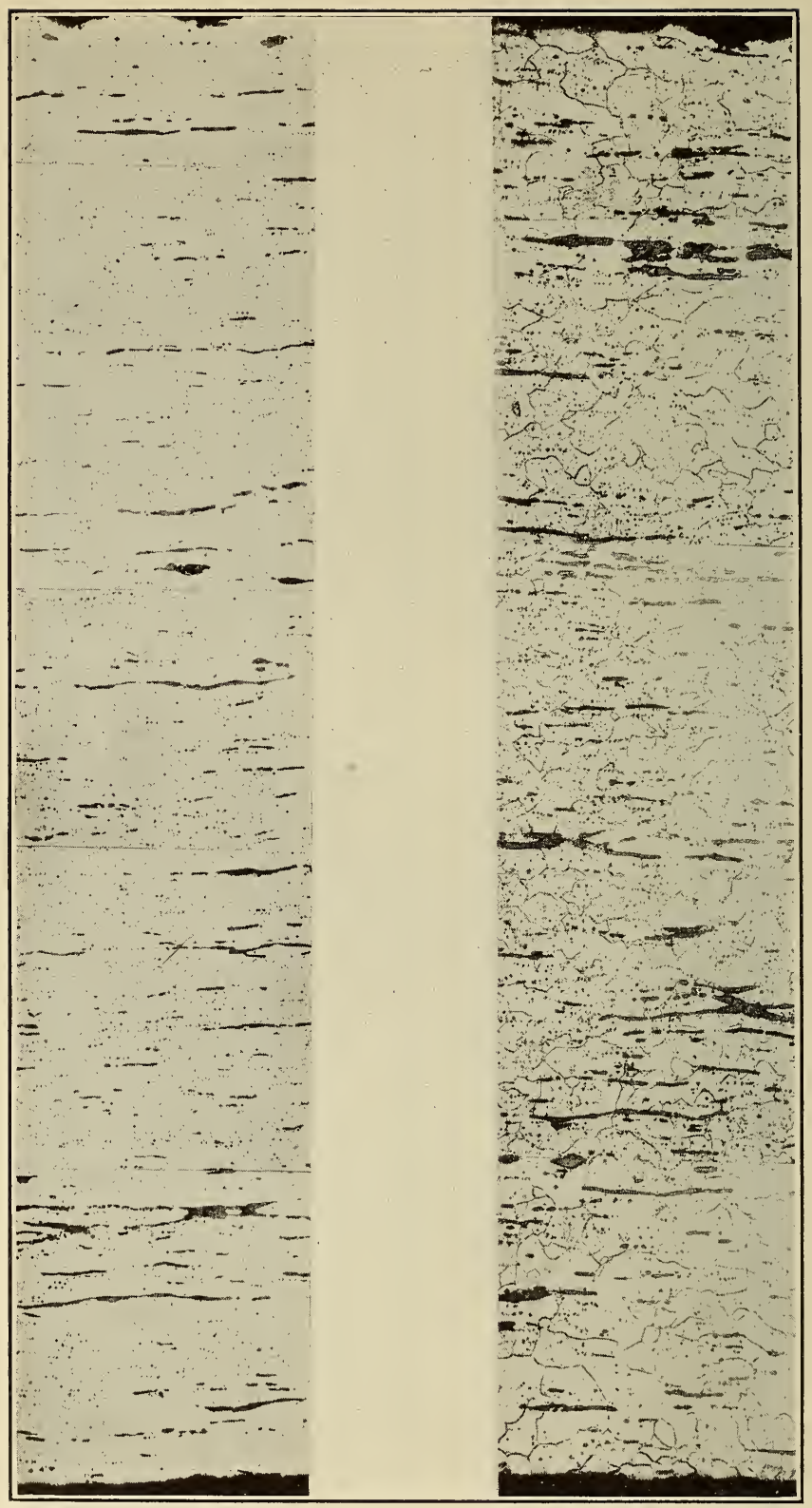

FIGURE 31.-Microstructure of wrought-iron pipe made by the Aston process, $\times 100$, reduced to $\times 50$

Longitudinal sections, unetched and etched, of the wall of a 1-inch pipe. 
B. S. Journal of Research, RPI24

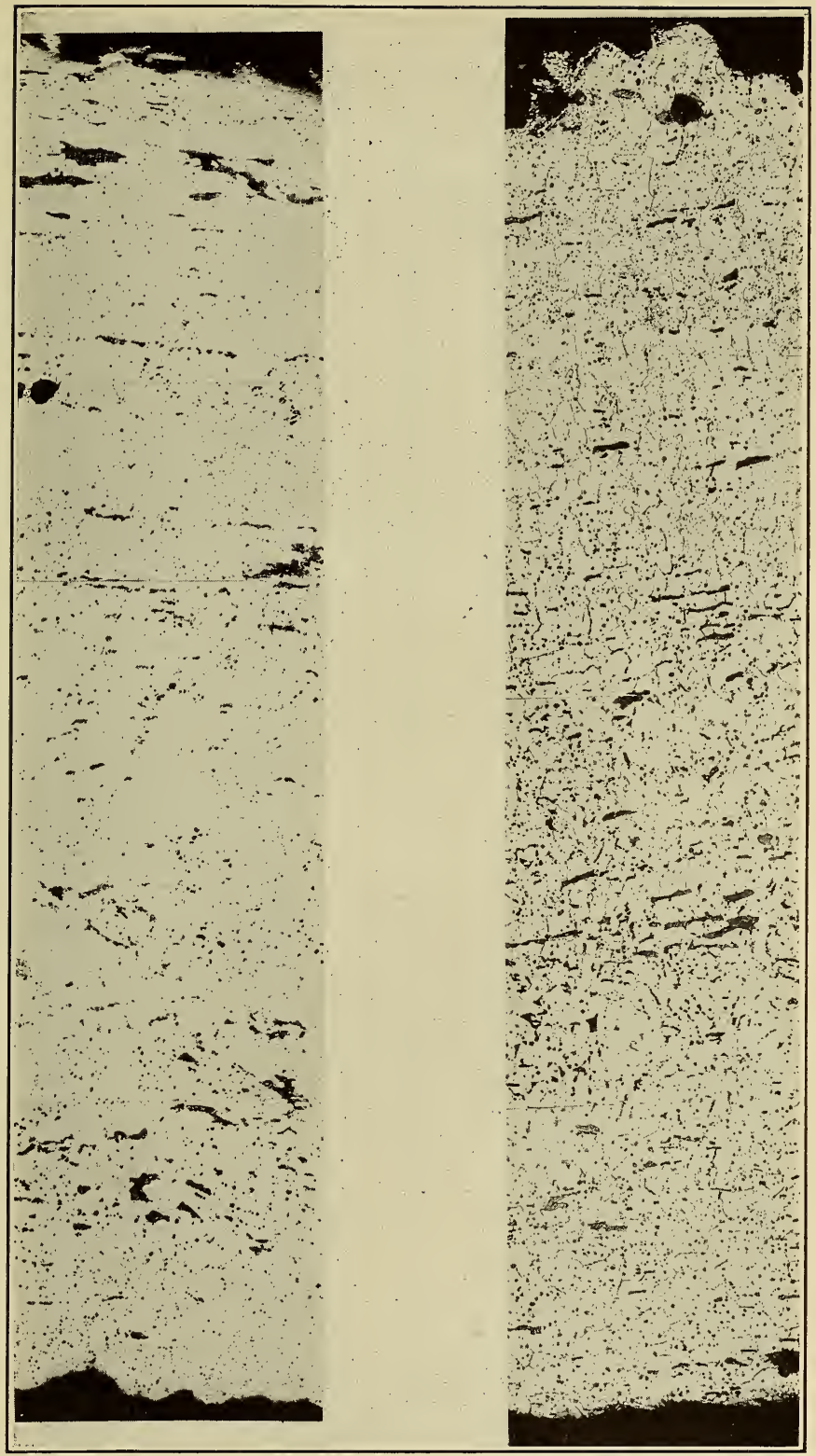

FIGURE 32.-Microstructure of wrought-iron pipe, $\times 100$, reduced to $\times 50$

Transverse sections, unetched and etched, of the wall of a 1-inch pipe made of the composite iron containing equal parts of hand-puddled and the new-process iron. 
B. S. Journal of Research, RP124

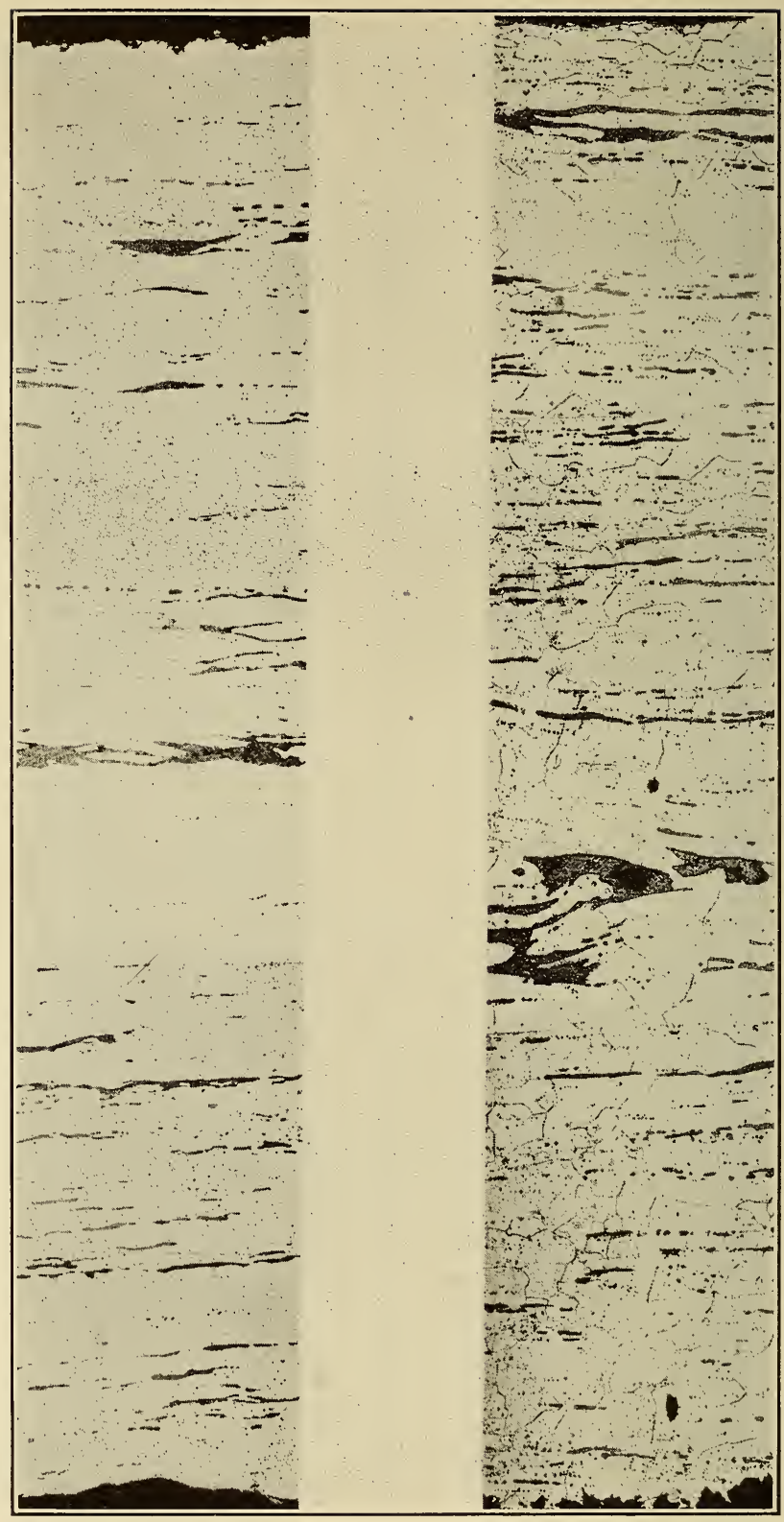

FIGURE 33.-Microstructure of wrought-iron pipe, $\times 100$, reduced to $\times 50$

Longitudinal sections, unetched and etched, of the entire wall of two pipes of the composite iron (equal parts of the two types of iron) 
(3) Skelp.-The examination of samples of the skelp taken from the front, middle, and back of the strip as rolled showed no important differences in the structure corresponding to these three positions. The average distribution of the slag threads, together with some of the largest slag threads found, as seen in the transverse sections of each of the three kinds of skelp, is shown in Figure 27. The examination of this material after etching showed considerably more uniformity with respect to carbon distribution in the hand-puddled product than was found in the muck bar and ball stages. This is a natural consequence of the heating which the piles formed from the sheared muck bar received, together with the rolling into skelp. The average carbon content of the streaks which were found was estimated -from the microstructure to be 0.15 to 0.20 per cent. A few of these streaks were found in the skelp rolled from the composite pile containing equal parts of the two types of iron.

(4) PIPE.-The structural condition of the iron after being worked into the form of pipe is depicted in Figures 28 to 33, inclusive. Transverse and longitudinal sections of the entire wall of the pipe are shown for each of the three kinds. There were no features found in any case which are not common in commercial wrought-iron pipe. The relatively cleaner appearance of the pipe made from the new iron and particularly the absence of "carbon streaks" is noteworthy. No very marked difference in the slag distribution in any of the three irons was noted, although the occurrence of relatively large slag threads in the hand-puddled iron was somewhat more frequent than in the comparison material.

\section{(b) 1-INCH ROUNDS}

The macrostructure of the wrought iron produced by the Aston process after being rolled into the form of 1 -inch rounds is shown in Figure 34. The effect of the repeated piling or refining which some of this material received is very evident. No unusual features in the microstructure of the iron were noted. The size of the slag threads naturally varied somewhat with the amount of piling and rolling which the iron received. So far as can be judged from the microstructure alone, as is shown in Figure 35, this material would be considered as high-grade wrought iron.

\section{(c) SLAG}

Wrought-iron slag is essentially a ferrous silicate slag. According to Herty and Fitterer, ${ }^{9}$ such slags containing silica in amounts less than 29 per cent contain two constituents, $\mathrm{FeO}$ and a silicate, fayalite $\left(2 \mathrm{FeO}-\mathrm{SiO}_{2}\right)$. The eutectic for this part of the system occurs

9 C. H. Herty and G. R. Fitterer, The Physical Chemistry of Steel Making: Deoxidation with Silicon and the Formation of Ferrous-Silicate Inclusions in Steel, Bull. 36, Min. \& Met. Investigations, Carnegie Institute of Technology and Bureau of Mines; 1928. 
with a silica content close to 21 per cent. Pure ferrous silicate slags containing less than 21 per cent silica, therefore, consist of excess $\mathrm{FeO}$ embedded in a eutectic matrix of $\mathrm{FeO}$ and fayalite. This is the structural condition which obtains in wrought-iron slags ordinarily. The eutectic is extremely fine and appears as a single constituent at the magnifications ordinarily used in studying the structure of wrought iron.

It will be noted (fig. 36 ) that slags representative of the product of hand puddling and of that of the new process have the same general structure and show no essential differences in structure such as would be considered significant of marked differences in properties. These samples, which were secured as drippings from the wroughtiron ball, were remelted in the laboratory in iron crucibles and allowed to cool slowly in the furnace so as to approach a condition of equilibrium.

Observations were made on the behavior of the two slags when heated to fusion, the samples being contained in small iron crucibles and heated in an Arsem vacuum furnace, the pressure within the furnace being somewhat less than that equivalent to $1 \mathrm{~mm}$ of mercury. Both slags melted quietly in the crucibles and there was no evidence of any loss by sputtering. A crucible containing handpuddled slag, which had been previously ground in a mortar, weighing $17.5 \mathrm{~g}$ (crucible $6.8 \mathrm{~g}$ ) weighed $17.3 \mathrm{~g}$ after heating. Similar weights for the slag of the new process were $17.9 \mathrm{~g}$ before heating (crucible, $6.9 \mathrm{~g})$ and $17.8 \mathrm{~g}$ after fusion of the slag.

Observations by means of an optical pyrometer of the "disappearing filament" type, of samples heated similarly to these showed that the new-process slag gave distinct visible evidence of melting at $1,280^{\circ}$ C. $\left(2,335^{\circ}\right.$ F. $)$ and was completely molten at $1,350^{\circ} \mathrm{C}$. $\left(2,460^{\circ}\right.$ F.). Upon slowly cooling, this slag appeared completely frozen at $1,270^{\circ}$ C. $\left(2,320^{\circ}\right.$ F. $)$. Slag from the hand-puddling process showed evidence of melting at $1,250^{\circ} \mathrm{C}$. $\left(2,280^{\circ} \mathrm{F}\right.$.) and was completely liquid qt $1,350^{\circ} \mathrm{C}$. $\left(2,460^{\circ} \mathrm{F}\right.$.). On cooling, it had the appearance of being completely frozen at $1,220^{\circ} \mathrm{C}$. $\left(2,230^{\circ} \mathrm{F}\right.$.).

Figure 37 shows the appearance of the surface of the two samples after being melted and allowed to freeze in the crucible. It will be noted that the slag from the hand-puddled process shows numerous shrinkage holes or "pores" over the surface which may be related to the evolution of gases during freezing. These were lacking in the comparison sample which froze with a continuous though slightly roughened surface.

A series of determinations of the relative viscosity of the two slags was made ${ }^{10}$ in the plant, the slag in each case being taken directly

\footnotetext{
${ }^{10}$ These determinations were made by members of the staff of the Bureau of Mines, Pittsburgh station.
} 
B. S. Journal of Research, RP124

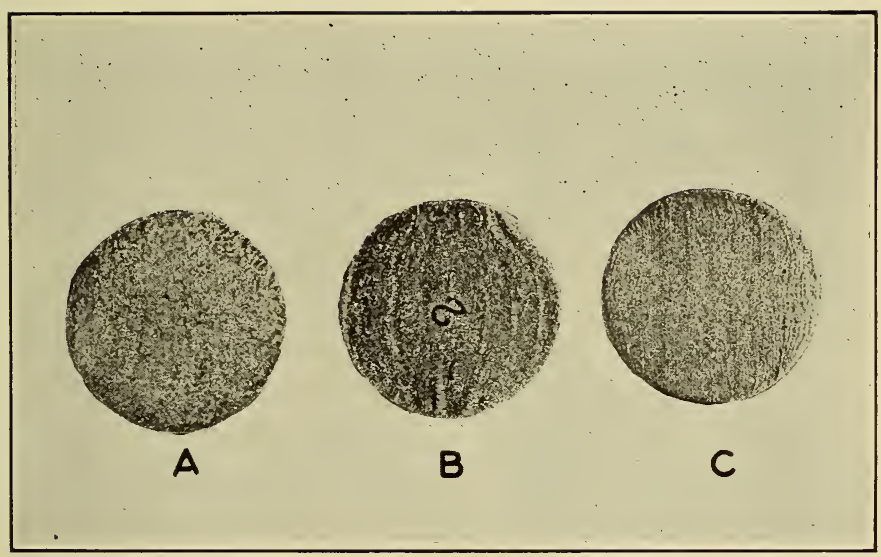

Figure 34.-Microstructure of the new-process wrought iron in 1 -inch rounds, $\times 3 / 4$

$a$, "Direct rolled"; $b$, "single refined"; $c$, “double refined." Etchant, 4 per cent nitric-acid solution. 


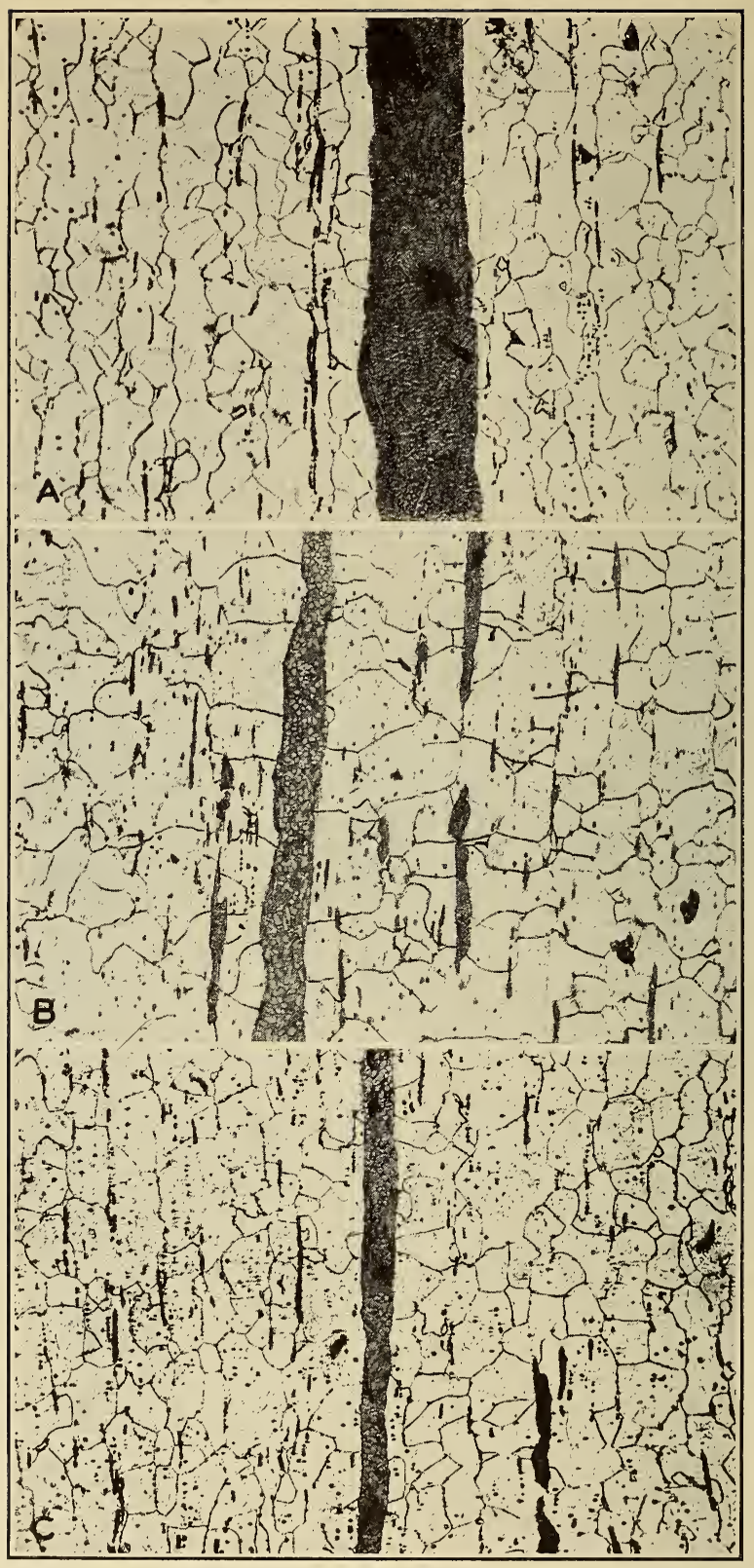

FIgURE 35.-Microstructure of the new-process wrought iron in 1-inch rounds, $\times 100$, reduced to $\times 75$

$a$, "Direct rolled" iron; $b$, "single refined" iron; $c$, "double refined," iron. The large slag threads illustrate the relative size of such threads in the three grades of iron. Such threads are only occasionally found. Etchant, alcoholic picric-acid solution. 


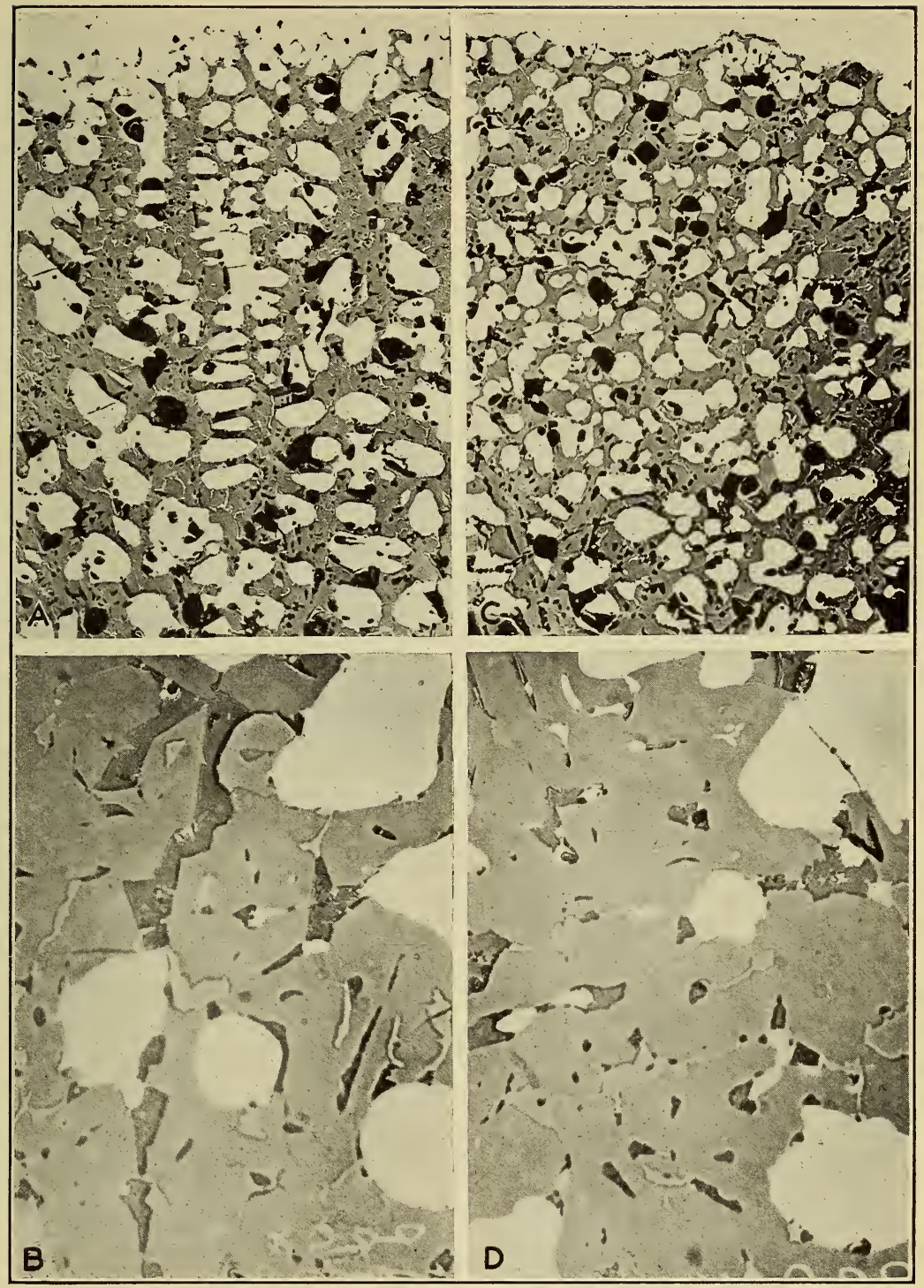

FIGURE 36.-Microstructure of wrought-iron slag, unetched

The slags obtained as drippings from the "ball" were melted in iron crucibles in a vacuum furnace and allowed to cool slowly. In both slags, the light areas are $\mathrm{FeO}$; the gray background is fayalite. Note, in $b$ and $d$, the other structural constituents embedded in the fayalite matrix. $a$, Slag from hand-puddled iron. The light portion at the top of the micrograph represents the iron crucible. Note how the slag has penetrated into it, $\times 100$, reduced to $\times 75 ; b$, Same as $a, \times 500$ reduced to $\times 375 ; c$, slag from the new-process iron, $\times 100$ reduced to $\times 75 ; d$, same as $c, \times 500$, reduced to $\times 375$. 
B. S. Journal of Research, RP124

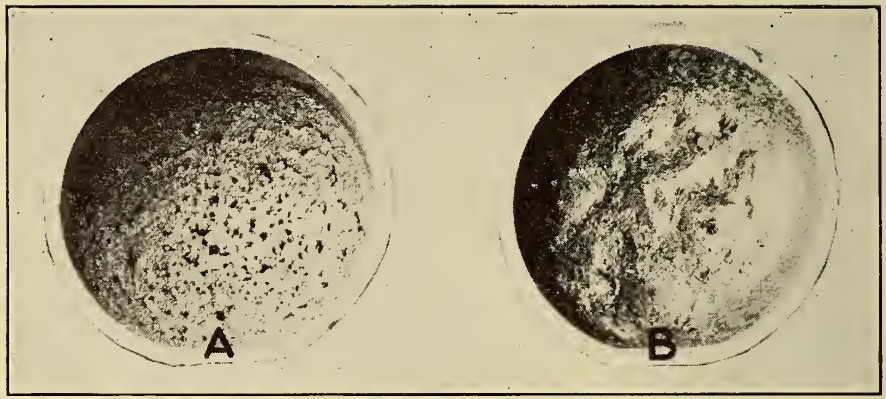

Frgure 37.- Surface appearance of wrought-iron slags after remelting followed by slow cooling, $\times 2$

The photographs show the surface of the slag after being melted and allowed to solidify in the crucible. $a$, Slag from hand-puddled iron; $b$, slag from new-process iron. 
either from the puddling furnace or from the "shotting" cup, for the new process. The inclined-plane method was used. The plane forms an angle of $30^{\circ}$ with the horizontal and the thickness of the layer formed as the molten slag flows down the plane was measured at a distance of 12 inches from the top of the plane. The results are summarized in Table 17. It will be noted that according to these results, the slag taken from the hand-puddling furnace was somewhat more viscous than that of the new process.

TABLE 17.-Relative viscosity of slag of the hand-puddling process and of the new process

[The inclined plane method was used. The thickness of the slag layer was measured 12 inches from top of the plane]

\begin{tabular}{|c|c|c|c|c|c|}
\hline Material & $\begin{array}{l}\text { Sam- } \\
\text { ple } \\
\text { No. }\end{array}$ & Condition of sampling & \multicolumn{2}{|c|}{ Temperature 1} & $\begin{array}{l}\text { Thick- } \\
\text { ness of }\end{array}$ \\
\hline \multirow{2}{*}{ Hand-puddled slag } & $\begin{array}{l}\mathrm{H} 1 \\
\mathrm{H} 2 \\
\mathrm{H} 3 \\
\mathrm{H} 4\end{array}$ & $\begin{array}{l}\text { During the boil } \\
\text { do do }\end{array}$ & $\begin{array}{l}{ }^{\circ} F \\
2,400 \\
2,400 \\
2,400 \\
2,400\end{array}$ & $\begin{array}{l}{ }^{\circ} C . \\
1,315 \\
1,315 \\
1,315 \\
1,315\end{array}$ & $\begin{array}{l}\text { Inch } \\
0.075 \\
.077 \\
.080 \\
.085\end{array}$ \\
\hline & $\begin{array}{l}\text { H5 } \\
\text { H6 } \\
\text { H7 }\end{array}$ & $\begin{array}{l}\text { Slag remaining in furnace after remov- } \\
\text { al of ball. }\end{array}$ & $\begin{array}{l}2,520 \\
2,520 \\
2,520\end{array}$ & $\begin{array}{l}1,380 \\
1,380 \\
1,380\end{array}$ & $\begin{array}{l}.072 \\
.070 \\
.075\end{array}$ \\
\hline New-process slag- & $\begin{array}{l}\text { A1 } \\
\text { A2 } \\
\text { A3 } \\
\text { A4 } \\
\text { A5 } \\
\text { A6 } \\
\text { A7 } \\
\text { A8 } \\
\text { A9 } \\
\text { A10 }\end{array}$ & $\begin{array}{l}\text { From slag thimble before shotting } \\
\text { From slag thimble after shotting } \\
\text { do }\end{array}$ & $\begin{array}{l}2,400 \\
2,400 \\
2,400 \\
2,400 \\
2,350 \\
2,400 \\
2,400 \\
2,400 \\
2,400 \\
2,400\end{array}$ & $\begin{array}{l}1,315 \\
1,315 \\
1,315 \\
1,315 \\
1,290 \\
1,315 \\
1,315 \\
1,315 \\
1,315 \\
1,315\end{array}$ & $\begin{array}{l}.045 \\
.044 \\
.052 \\
.065 \\
.072 \\
.042 \\
.043 \\
.057 \\
.051 \\
.042\end{array}$ \\
\hline
\end{tabular}

1 Temperatures are only approximate.

Wrought-iron slags always contain other substances besides $\mathrm{FeO}$ and $\mathrm{SiO}_{2}$. (Table 4.) A considerable amount of the iron may be in the ferric condition $\left(\mathrm{Fe}_{2} \mathrm{O}_{3}\right)$ and phosphorus is always present as well as some other constituents. Evidence of other structural constituents in addition to $\mathrm{FeO}$ and fayalite can practically always be found in such slags. (Fig. 36.) No difference in the two slags were noted in this respect.

\section{MISCELLANEOUS TESTS AND OBSERVATIONS}

(a) OBSERVATIONS ON BLISTERING

It is well known that "blisters" are quite common on the inner surface of wrought-iron pipe of the smaller sizes. In cutting up the pipe lengths used in this study which had been chosen at random without any inspection, whatsoever, a few blistered areas were noted and in such cases that portion of the pipe was not used for test specimens. It is of interest to note that such blisters were confined almost entirely to the pipe made from the hand-puddled iron. Undoubtedly 
the formation of these blisters is associated with the occasional "carbon streaks" which occur in this type of iron and is to be attributed to the liberation of gas $(\mathrm{CO})$ in the reaction between the combined carbon and the ferrous oxide of the slag. The fact that the iron made by the new process has a very low carbon content, (rarely over 0.03 per cent), in all stages of fabrication, from the ball to the finished pipe, is believed to account for the relatively high degree of freedom of this type of pipe from this feature. Figure 38 shows the appearance of some of the blistered areas.

\section{(b) BEHAVIOR ON CARBURIZING}

It has been demonstrated beyond all reasonable doubt ${ }^{11}$ that, by means of carburizing such as is done in the common casehardening process, certain differences in steel can be shown which are detectable in practically no other manner. It appeared of interest, therefore, to apply this test, which is ordinarily referred to as the "McQuaidEhntest," to the two types of iron. Samples of the muck bar and skelp of both the hand-puddled iron and the comparison iron made by the new process were carburized by being heated for eight hours at $940^{\circ} \mathrm{C}\left(1,725^{\circ} \mathrm{F}\right.$.), while embedded in a commercial carburizing compound and then being allowed to cool in the furnace. The microscopical examination ${ }^{12}$ revealed no essential difference in the structure of the carburized irons. Both irons showed a very "abnormal" structure, the term being used in the same sense as it is applied to carburizing steels.

\section{(c) MILL OBSERVATIONS}

"Weldability" and ease of machining rank high among the desirable characteristics of wrought iron. Observations on the relative weldability of the two types of iron as it was being made into pipe were made in the mill, together with the usual flattening and hydrostatic tests which form part of the routine mill tests. The newprocess iron behaved in all essential respects in these tests like the hand-puddled iron. Such visual observations as it was possible to make on the machining of the pipe in the mill indicated no difference in the behavior of the two irons during the threading of the ends of the pipe. Likewise the observations made on the behavior of the two during galvanizing, supplemented by flattening tests of the galvanized pipe, indicated that the "galvanizing properties" of the new iron are equal in all respects to those of the hand-puddled product.

11 S. Epstein and H. S. Rawdon, Steel for Case Hardening-Normal and Abnormal Steel, Bureau of Standards Journal of Research, 1, 423, 1928; Research Paper No. 14.

12 This test was carried out by Samuel Epstein, associate metallurgist. 


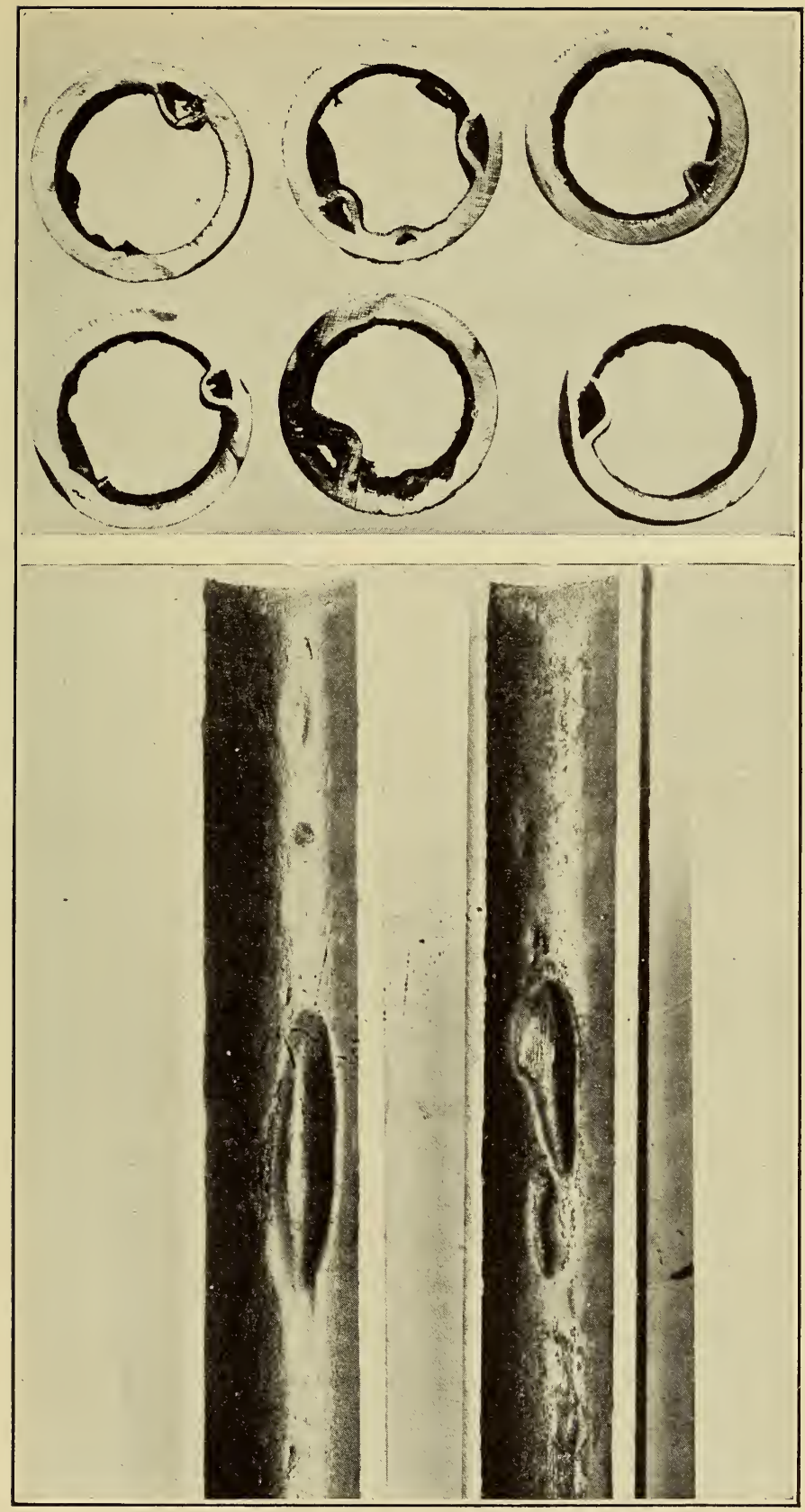

Figure 38. - "Blisters" in wrought-iron pipe

The transverse and longitudinal sections show the blistered appearance of the inner pipe surface which is frequently observed. 


\section{DISCUSSION}

Wrought iron is universally conceded to be a malleable product formed from the refining of pig iron (or ore) by the almost complete elimination of the metalloids in the material and, in the wrought state, has a characteristic structure referred to as "fibrous," which is the result of the intimate mechanical mixture of some slag with the iron. Since wrought iron is a mixture of two unlike substances, its composition, at least as determined by ordinary chemical analysis can not readily be used as a basis of a definition as may be done in the case of steel. The refining of tha raw material must necessarily be quite complete in order to obtain a malleable product. The metalloids initially present in the pig iron, if not entirely eliminated, are reduced to a very small percentage. Aside from the restriction concerning the maximum percentage of manganese permitted, for example, 0.10 per cent,${ }^{13}$ chemical composition is not usually specifically mentioned in specifications for wrought iron. In the results of the chemical analyses of the different irons summarized in Table 3 , nothing will be found which could be used as a basis of adverse criticism of any of the irons. It will be noted that the carbon content of the new-process iron was lower than that of the comparison iron, and, as shown by the metallographic examination, this product showed no nonuniformity such as would result from "carbon streaks." It should not be inferred, however, that nonuniformity by the presence of carbon streaks is necessarily a characteristic of all wrought iron made by the hand-puddling process.

On the basis of the density measurements, there is little to be said concerning the possible differences in the two types of iron. As might be predicted, the iron in the form of muck bar was somewhat less dense than after being rolled into skelp or pipe, the hand-puddled muck bar showing a slightly lower density than the comparison iron. This was also true in the case of the pipe and skelp, the differences were so slight, however, as to appear to be of no practical significance.

For purposes of comparison, the average tensile properties of the pipe materials have been summarized in Figure 39. The properties of the iron in the form of muck bar, are of course, of no practical concern; the fact is of some interest, however, that iron made by the new process even in this rough form, showed considerably higher ductility, though with a somewhat lower tensile strength, than the hand-puddled iron did. The presence of combined carbon in the latter in sufficient amounts as to form easily recognized "carbon streaks" is probably the reason for this difference. The working

${ }^{13}$ Specifications A84-27 and A86-27, Am. Soc. Test. Mtls. Standards; 1927. 
which the materials received in putting them into the form of skelp and pipe improved the tensile properties. It will be noted, however, that, in general, the hand-puddled iron after the additional rolling

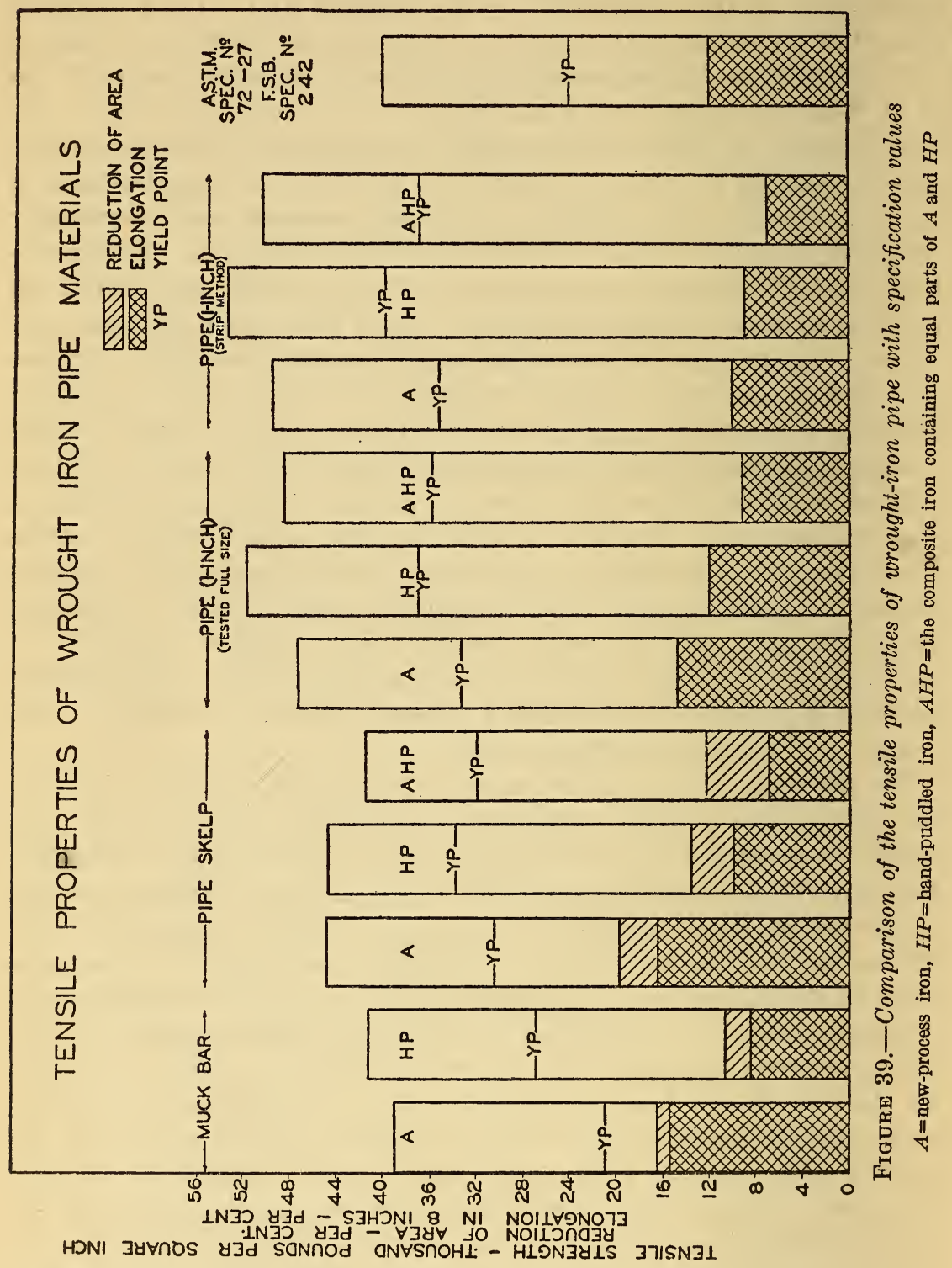

still showed considerably higher tensile strength, accompanied by lower ductility, than did the comparison wrought iron.

By comparison with the tensile properties for wrought-iron pipe of this size as required by specifications of the American Society for 
Testing Materials and the Federal Specifications Board, ${ }^{14}$ it will be seen that both the hand-puddled wrought iron and the new process wrought iron when tested as full-size specimens, would meet the required tensile properties. However, when the tensile properties were determined on longitudinal strips machined from the pipe, as is permitted by current specifications, the average elongation was found to be lowered very considerably. This lowering of the elongation is to be attributed to the form of the test specimen and not to any inherent property of the iron. The specimen, in each case, represented the full wall thickness and the intitial curvature was maintained through the test. On the bases of these tests, it would appear that in testing pipe of small diameter, the strip method, at least as carried out here, is not suitable and should not be depended upon. ${ }^{15}$

The torsional properties of wrought iron are not ordinarily taken into account in specifications for pipe. For rotary drill pipe, of course, the behavior of the pipe under torsion may be of considerable importance. The torsion tests, in the present study, however, afforded another convenient means for comparing the properties of the two irons, in the form of pipe. It will be noted (Table 10) that the results of the torsional tests confirmed those of the tension tests in showing that the hand-puddled iron used was a slightly stronger, but somewhat less ductile material than the comparison wrought iron.

The properties of the new iron when rolled into the form of 1-inch rounds compare favorably with those of high-grade hand-puddled wrought iron made commercially in this form. The tensile properties and impact resistance are summarized in Figure 40, together with the corresponding properties of 1-inch rounds of high-grade wrought iron which were obtained in a previous series of tests. ${ }^{16}$ It has already been stated that the 1-inch rounds used in the present study were especially rolled for these tests under regular mill conditions. The comparison materials represent wrought-iron rounds made to meet certain specifications of the American Society for Testing Materials.

It is evident from the results of nearly all of the tests carried out upon the 1-inch rounds of the new-process iron (Tables 12, 13, 14, and 15) that the amount of working, that is, refining (piling and reheating) which the iron received did not affect its properties to a very marked extent. The tensile, torsional, and "single-blow notched-bar" impact resistance properties were found not to differ

\footnotetext{
14 Standard specifications A72-27, Am. Soc. Test. Mtls. Standards, 1927. Also Federal Specifications Board, specification 242, "Wrought Iron Pipe, Welded (Black and Galvanized); 1925.

15 Specification 242 of the Federal Specifications Board is now being amended in this respect, the question having been brought up for consideration by another manufacturer of wrought-iron pipe. For the determination of the tensile properties of pipe 2 inches in diameter and less, the tension specimens consist of fullsize pipe sections, the strip method being restricted to the pipe of larger diameter.

16 See footnote 4, p. 954.
}

$77886^{\circ}-29-11$ 
greatly for the "direct-rolled," the "single-refined," and the "doublerefined" irons. To supplement the impact tests (single-blow notched-

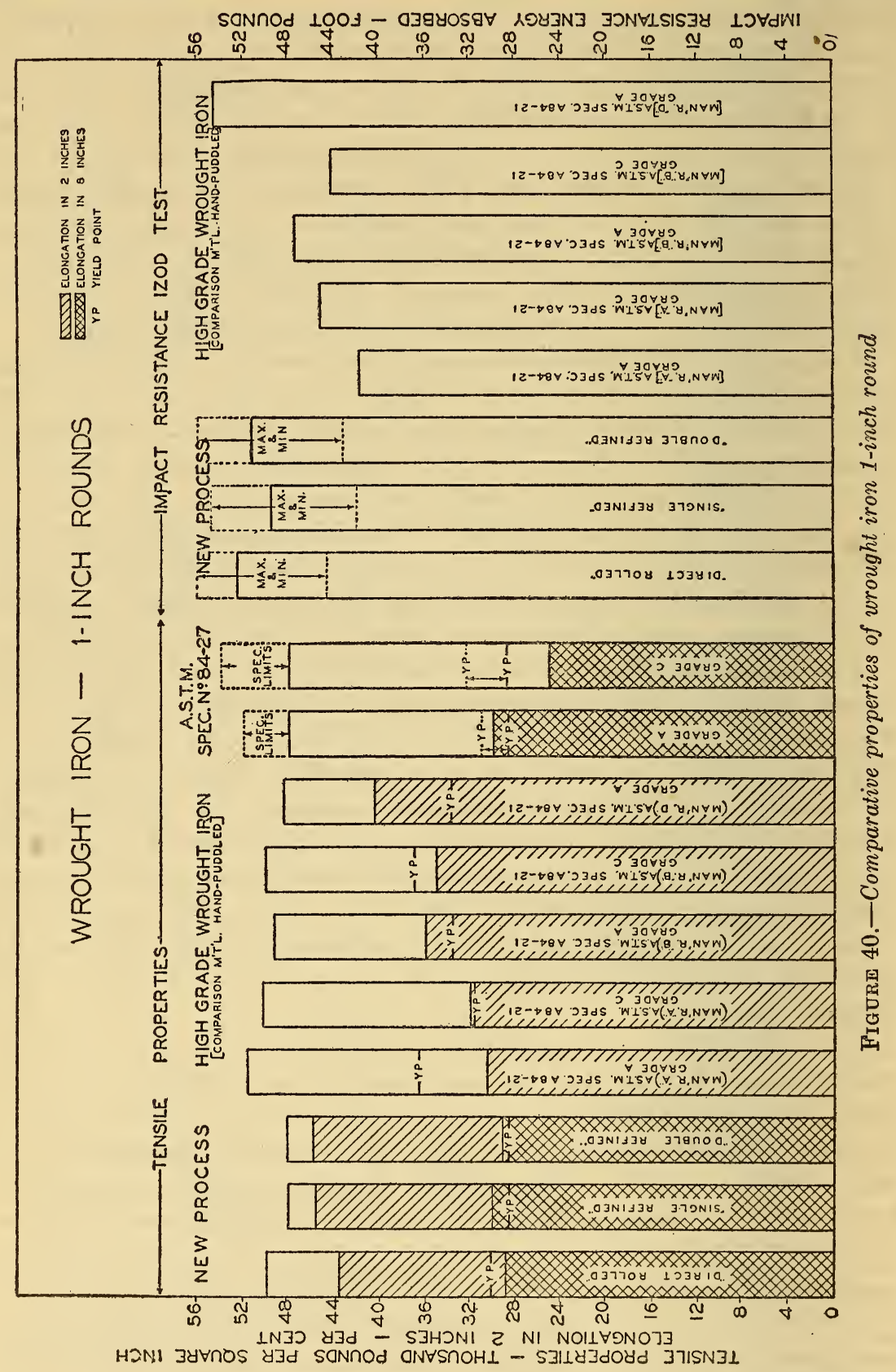

bar), the ends of the specimens were etched, after the test, to show the macrostructure in order to find out how the blows had been struck 
with respect to the piling. In the majority of cases, the maximum resistance was shown when the blow was struck against the face of the "slabs." However, it was noticed that, when the three blows for any one specimen were struck at $45^{\circ}$ to the face of the slabs, as was the case for a number of specimens, the difference between the maximum and minimum was as great, in some instances, as when the blows struck the face and edge of the slabs. It would appear, however, from the results obtained in the repeated-blow impact tests (Table 15) that the impact resistance of the iron against repeated blows was noticeably improved by the additional "refining" which was given the iron.

In carrying out the corrosion tests no attention whatsoever was given to the controversial question as to the relative corrodibility of wrought iron and steel. The sole purpose of the tests was to show whether or not the corrosion behavior of the two types of iron, in the form of pipe, differed to any marked extent. A comparison of the results for the wrought iron made by the Aston process with those for the hand-puddled product indicates a very slightly higher corrosion resistance for the latter. These indications are in accord with the results of the tests carried out to show the relative electrolytic solution potential of the two. These tests indicated that the new-process iron, when in electrical contact with the hand-puddled iron, both being immersed in a solution, behaves as the anode of the couple; that is, it has a higher solution pressure. It might be argued therefore, that it might be unwise to use the two different kinds together. The results obtained with the pipe made from the composite iron, which was rolled from an 8-high pipe of muck bar composed of equal amounts of the two types of iron did not confirm this prediction.

As might be expected, the method of the corrosion test had a marked influence on the apparent corrosion resistance of the irons. The average sorrosion loss obtained in simple immersion in tap water or in $3 \frac{1}{2}$ per cent sea-salt solution (unaerated) was in the neighborhood of 0.006 to $0.007 \mathrm{~g} / \mathrm{dm}^{2} / \mathrm{day}$, whereas the loss for the same irons in the same solutions, when aerated for 8 hours of every 24 , was approximately four to five times as great $\left(0.021\right.$ to $0.034 \mathrm{~g} / \mathrm{dm}^{2} /$ day). In the wet-and-dry corrosion tests, the loss was much more pronounced ( 0.125 to 0.168 and 0.05 to $0.07 \mathrm{~g} / \mathrm{dm}^{2} /$ day in sea-salt solution and tap water, respectively). It is evident that the effect, on the corrosion behavior of these materials, of the conditions under which the corrosive attack was brought about far overshadowed possible effects resulting from differences in the materials.

Of the different corrosion methods used, the immersion tests, without question, are more nearly representative of conditions for pipes in service than the wet-and-dry tests are. And of the two types of immersion tests, those carried out in tap water which was not exces- 
sively aerated prokably represent service conditions for the large majority of pipe better than the other immersion tests. It is evident from Figure 15, that on the basis of the results obtained with the simple immersion tests in either of the liquids used, no sharp distinction as to the relative merits of the various materials can be drawn. Likewise, the average corrosion losses obtained in the immersion tests in highly aerated liquids, especially those in tap water, do not differ sufficiently to warrant sharp distinctions being drawn as to the relative corrosion resistance of three materials.

Concerning the similarity in structure of the two types of iron, little need be said. The micrographs clearly show that the product made by the new process has the structural features which are generally considered to be characteristic of wrought iron. Indeed, it is extremely doubtful whether any metal microscopist could distinguish with certainty a sample of the new wrought iron from iron made by the familiar hand-puddling process if the sample were submitted to him as an "unknown." The almost complete absence of carbon streaks from the structure of the iron made by the new process is a noteworthy feature and any possible distinction between the two irons from the standpoint of structure would undoubtedly have to be drawn on this basis.

The examination of the slag from the two different processes showed that both were essentially ferrous silicate slags containing an excess of iron oxide, although not absolutely identical in either chemical composition or structure. The slight differences, however, would not seem to be of importance from a practical standpoint so far as the properties and use of the finished product are concerned.

In the observations made in the mill, especially on the welding, galvanizing, and machining properties of the two types of iron in the form of pipe, nothing was seen to indicate any marked differences in the two irons in these respects.

\section{SUMMARY}

1. A comparison was made of the properties and structure of wrought iron made by the hand-puddling process with those of wrought iron produced by a new process. The new process differs radically from the conventional hand-puddling process in that the pig iron after being melted in a cupola is refined in a Bessemer converter and then incorporated with the slag which has been prepared in a separate furnace. The "shotting" operation by which the slag is intimately mixed with the iron consists in pouring the molten metal into a bath of the molten slag. The resulting "ball" after being squeezed into a bloom is rolled in the ordinary manner into the desired shape. The comparison tests were carried out largely upon commercial 1-inch butt-welded black pipe, since, as yet, the commer- 
cial use of the new process is confined almost entirely to pipe. Three types of pipe were used, hand-puddled iron, new-process iron, and a "composite" iron composed of equal parts of the other two. Tests were also made upon the muck bar and skelp from which the pipe was rolled. The samples were chosen at random in the mill, and each length was sampled so as to obtain samples representative of the front, middle, and back, as rolled, of the strips selected. All of the succeeding tests were made on all of these samples. In addition to pipe materials, tests were made upon the new-process wrought iron in the form of 1-inch rounds, rolled especially for this work.

2. The chemical composition of the iron made by the new process was found to agree closely with that of the hand-puddled iron. The carbon content was consistently lower in the new-process iron than in the comparison iron and more uniform in that "carbon streaks" were entirely absent in the structure as shown by metallographic examination. The sulphur content of the new-process iron was not quite so low as that of the hand-puddled product.

3. No marked differences in density were found in the irons. The new-process iron had a slightly higher density, but the difference was so slight as to appear to be of no practical significance.

4. No marked difference in mechanical properties were found in the two irons, in pipe form, as shown by tension, torsion, and flattening tests. The iron made by the Aston process, on the whole, showed a slightly lower tensile strength accompanied by a higher ductility. Pipe made from the new-process iron meets the requirements of current specifications for pipe of the American Society for Testing Materials and the Federal Specifications Board.

5. In the form of 1-inch rounds the wrought iron made by the new process was found to compare very favorably in mechanical properties with standard grades of wrought iron of this form. The properties studied included tensile and torsion properties and the resistance of notched bars to single-blow and repeated-blow impact. Except in the "notched-bar repeated-blow" impact resistance, no important differences were noted in the different amounts of "refining" which the rounds received. The material used was representative of "direct-rolled," "single-refined," and "double-refined" iron.

6. The accelerated laboratory corrosion tests indicated very slight differences in the corrosion resistance in favor of the handpuddled iron. These differences were extremely slight as compared with the differences in corrosion behavior resulting from the different test methods used.

7. The structural features of the iron made by the Aston process are those which are generally conceded to be characteristic of wrought iron in general. The absence of "carbon streaks" in the newprocess iron is noteworthy. It is not to be concluded, however, 
that this feature is necessarily always present in hand-puddled iron. The puddling process is not nearly so favorable for the complete elimination of carbon, however, as is the new process. The occurrence of occasional relatively large slag threads in the hand-puddled iron was more frequent than in the comparison material according to the examination made.

8. Although not a primary object of this investigation, general observations made of the hand-puddling process and of the Aston process seemed to indicate a close parallelism in the important reactions entering into the manufacture of wrought iron in the two cases. The similarity of the products of the two processes confirm this indication.

9. In the observations made in the mill nothing was observed that would indicate that the behavior of the new-process wrought iron in welding, galvanizing, and machining is different from that of the wrought iron with which it was compared.

In addition to the acknowledgments made throughout the text, the authors wish to express their indebtedness to C. E. Eggenschwiler, junior laboratory assistant, for his very efficient help in a great many of the tests reported.

Washington, July 2, 1929. 